\title{
Estudos Teóricos dos Efeitos de Solvente no Espectro Eletrônico de Absorção da Molécula Óxido Mesitil.
}

\author{
Marcus Vinícius Araújo Damasceno
}

Dissertação apresentada ao Instituto de Física para a obtenção do título de Mestre em Ciências

Orientadora: Prof. ${ }^{a}$ Kaline Rabelo Coutinho

Banca Examinadora:

Prof. ${ }^{a}$ Kaline Rabelo Coutinho (IF/USP)

Prof. Lucy Vitória Credidio Assali (IF/USP)

Prof. Antônio Carlos Borin (IQ/USP)

Financiado pelo CNPq

São Paulo, 2009 


\section{FICHA CATALOGRÁFICA}

Preparada pelo Serviço de Biblioteca e Informação do Instituto de Física da Universidade de São Paulo

Damasceno, Marcus Vinícius Araújo

Estudos Teóricos dos Efeitos de Solvente no

Espectro Eletrônico de Absorção da Molécula Óxido Mesitil São Paulo, 2009.

Dissertação (Mestrado) - Universidade de São Paulo. Instituto de Física. Depto. de Física Geral

Orientador: Prof. ${ }^{a}$ Dra Kaline Coutinho

Área de Concentração: Física

Unitermos: 1. Física Computacional;

2. Mecânica Estatística Clássica;

3. Mecânica Quântica;

4. Estrutura Eletrônica;

5. Propriedades da Solução

USP/IF/SBI-096/2009 
"Dedico este trabalho à minha família pelo apoio e pela solidariedade e à minha avó Maria (in memoriam)." 


\section{Agradecimentos}

Gostaria de agradecer à todos que direta ou indiretamente contribuiram para a realização deste trabalho;

À Prof. ${ }^{a}$ Kaline Rabelo Coutinho, pela orientação, pelo agradável convívio e pela oportunidade de realizar este trabalho;

À toda minha familia, em especial aos meus pais Raimundo Damasceno e Maria de Lourdes e à minha irmã Márcia Rejane donde sempre obtive um apoio incondicional;

Ao grupo de Modelagem Molecular, coordenado pelos professores Sylvio Canuto e Kaline Coutinho, pelas discussões nos seminários que tanto contribuiram para a realização deste trabalho;

Aos amigos do DFGE pelas conversas e discussões, em especial, aos companheiros de sala Antonio, Alexandre e Evanildo e aos amigos do grupo, Carlos, Rodrigo, Evandro, Tiago, Rafael Barreto, Rafael Barroso, Thais, Fernando, Cintia, Yoelvis, Marcelo, Lucas, Paula;

Aos amigos distantes, em especial, Júnior, Alfredo, Olímpio, Lenilson, pelo incentivo;

Ao $\mathrm{CNPq}$, pelo apoio financeiro na execução deste trabalho. 



\section{Resumo}

Efeitos de solventes tem sido um tema de grande interesse científico. Em particular, o estudo dos efeitos de solventes no espectro eletrônico de absorção tem sua própria motivação e complexidade. Neste trabalho, nós estudamos os efeitos da solução aquosa na estabilidade conformacional e no espectro eletrônico de absorção da molécula Óxido Mesitil (OM). Essa molécula pertence a família das cetonas $\alpha, \beta$-insaturadas e, semelhantemente aos outros membros da família, ela apresenta transições eletrônicas sensíveis ao solvente.

Inicialmente, estudamos os isômeros syn e anti do OM isoladamente usando cálculos quânticos para determinar a energia livre relativa, a barreira de rotação, os momentos de dipolo e as transições eletrônicas de absorção. Nosso melhor resultado mostra que o isômero syn do OM é a conformação mais estável, por cerca de $1.3 \mathrm{kcal} /$ mol calculado com nível MP2/aug-cc-pVDZ. Com o mesmo nível de cálculo, obtivemos os momentos de dipolo de 2.80 e $3.97 \mathrm{D}$ para os isômeros syn e anti respectivamente, que estão em boa concordância com os valores experimentais de 2.8 e 3.7 D. Para o espectro eletrônico de absorção, analisamos a banda mais intensa, $\pi-\pi^{*}$, com diferentes funcionais de densidade e funções base. Obtivemos o comprimento de transição de 229 nm calculado com nível TD-B3LYP $/ 6-311++\mathrm{G}^{* *}$ para o isômero syn em muito boa concordância com o valor experimental de $231 \mathrm{~nm}$ medido em solução de iso-octano (solvente de baixa polaridade).

Para realizar os estudos em solução, geramos estruturas supermoleculares dos isômeros do OM em solução aquosa usando simulações computacionais com o método Monte Carlo. Usamos os potenciais Lennard-Jones e Coulomb para descrever as interações intermoleculares com os parâmetros do campo de força OPLS. Verificamos que as cargas atômicas OPLS não descrevem bem o potencial eletrostático do OM. Portanto, realizamos um processo iterativo para incluir a polarização do soluto na presença do solvente para descrever melhor as interações entre o OM e as moléculas de água. Assim, obtivemos um aumento de cerca de $80 \%$ nos momentos de dipolo dos isômeros isolados. Adicionalmente, calculamos a energia livre relativa entre os isômeros em solução aquosa usando teoria de perturbação termodinâmica. Obtivemos que o isômero anti do OM é a conformação mais estável, por cerca de $2.8 \mathrm{kcal} / \mathrm{mol}$. 
Examinando os efeitos de solvente no espectro eletrônico de absorção do OM, identificamos que existem duas contribuições competindo para o deslocamento da banda $\pi-\pi^{*}$. Uma contribuição vem da mudança conformacional syn $\rightarrow$ anti do OM devido a mudança de polaridade, baixa $\rightarrow$ alta, do solvente. Essa mudança conformacional provoca um deslocamento para o azul de $1210 \mathrm{~cm}^{-1}$ na transição $\pi-\pi^{*}$. A outra contribuição vem do efeito do solvente na estrutura eletrônica do OM, que provoca um deslocamento para o vermelho de $-4460 \mathrm{~cm}^{-1}$ nessa transição. Adicionando essas duas contribuições, temos o efeito do solvente total no espectro eletrônico de absorção do OM em solução aquosa. Nosso melhor resultado é um valor médio de $248 \mathrm{~nm}$ obtido com 75 cálculos TD-B3LYP $/ 6-311++\mathrm{G}^{* *}$ de estruturas supermoleculares estatisticamente descorrelacionadas compostas por um anti-OM rodeado por 14 moléculas de água explícitas embebidas no campo eletrostático de 236 moléculas de água tratadas como cargas pontuais simples. Esse resultado está em muito boa concordância com o resultado experimental de $243 \mathrm{~nm}$ em solução aquosa. Sendo assim, este trabalho demonstra que a mudança conformacional syn $\rightarrow$ anti é essencial para entender o deslocamento espectral da transição $\pi-\pi^{*}$ do OM em água. 


\section{Abstract}

Solvent effects have been the subject of considerable scientific interest. In particular, the study of solvent effects in electronic absorption spectroscopy has its own motivation and complexities. In this work we study the effects of the aqueous solution in the conformational stability and the electronic absorption spectrum of the Mesityl Oxide (OM) molecule. This molecule belongs to the family of the $\alpha, \beta$-unsaturated ketones and, like other members of the family, presents sensitivity to solvent in the absorption transitions.

Initially we studied the isolated syn and anti isomers of OM by performing quantum mechanical calculations to obtain the relative free energy, the rotational barrier, the dipole moments and the electronic absorption transitions. Our best result showed that the OM syn isomer is the most stable conformer, by approximately $1.3 \mathrm{kcal} / \mathrm{mol}$ calculated with the MP2/aug-cc-pVDZ level. With the same level of calculation, we obtained the dipole moments of 2.80 and $3.97 \mathrm{D}$ for the syn and anti isomers respectively, which are in good agreement with the experimental values of 2.8 and $3.7 \mathrm{D}$. For the electronic absorption spectrum, we analyzed the most intense band, $\pi-\pi^{*}$, with different density functional and basis sets. We obtained a transition wavelength of $229 \mathrm{~nm}$ calculated with TD-B3LYP $/ 6-311++\mathrm{G}^{* *}$ level for the syn isomer in good agreement with the experimental value of $231 \mathrm{~nm}$ measured in iso-octane (solvent of low polarity).

For performing the in-solution studies, we generated supermolecular structures of the OM isomers in aqueous solution using computer simulations with the Monte Carlo method. We used the Lennard-Jones and Coulomb potentials to describe the intermolecular interactions with the OPLS force field parameters. We found that the OPLS atomic charges do not describe well the electrostatic potential of OM. Therefore we performed an iterative process for including the solute polarization in the presence of the solvent to better describe the interactions between the $\mathrm{OM}$ and the water molecules. We obtained an increase of about $80 \%$ in the dipole moments of the isolated isomers. Additionally, we calculated the relative free energy between the isomers in aqueous solution using thermodynamic perturbation theory. We found that the anti isomer is the most stable conformer in aqueous solution, by about $2.8 \mathrm{kcal} / \mathrm{mol}$. Examining the 
solvent effects in the electronic absorption spectrum of $\mathrm{OM}$, we found that there are two competing contributions to the $\pi-\pi^{*}$ band shift. One contribution is due to the syn $\rightarrow$ anti conformational change of OM caused by the low $\rightarrow$ high polarity change of the solvent. This conformational change led to a blue shift of $1210 \mathrm{~cm}^{-1}$ in the $\pi-\pi^{*}$ band. The remaining contribution is due to the solvent effect in the electronic structure of OM, which led to a red shift of $-4460 \mathrm{~cm}^{-1}$. Adding these two contributions, we obtained the total solvent effect in the electronic absorption spectrum of $\mathrm{OM}$ in aqueous solution. Our best result was an average wavelength transition of $248 \mathrm{~nm}$ obtained using 75 TD-B3LYP $/ 6-311++\mathrm{G}^{* *}$ quantum calculations on statistically uncorrelated supermolecular structures composed by one anti-OM surrounded by 14 explicit water molecules in the electrostatic embedding composed of 236 water molecules described as simple point charges. This result is in very good agreement with the experimental result of $243 \mathrm{~nm}$ in aqueous solution. Thus, this work demonstrates that the syn $\rightarrow$ anti conformational change is the essential ingredient to understand the spectral shift of the $\pi-\pi^{*}$ absorption transition of $\mathrm{OM}$ in water. 


\section{Sumário}

Lista de Abreviaturas $\quad$ x

Lista de Figuras $\quad$ xiv

Lista de Tabelas $\quad$ xix

\begin{tabular}{ll|l}
1 & Introdução & 1
\end{tabular}

1.1 Polaridade de Solventes . . . . . . . . . . . . . . . . . 4

1.2 Modelos para o Solvente . . . . . . . . . . . . . . 11

2 Métodos Quânticos 15

2.1 Introdução . . . . . . . . . . . . . . . . . . . 15

2.2 Aproximação de Born-Oppenheimer $\ldots \ldots \ldots$. . . . . . . 16

2.3 Método de Hartree-Fock . . . . . . . . . . . . . . . . . . . . . . . . . . 19

2.3 .1 Equação de Hartree-Fock-Roothaan . . . . . . . . . . . . . . 24

2.3 .2 Funções de Base . . . . . . . . . . . . . . . . . 27

2.4 Aproximações Perturbativas . . . . . . . . . . . . . . . . . . . . 29

2.4 .1 Teoria de Perturbação de Rayleigh-Schrödinger . . . . . . . . . 29

2.4 .2 Teoria de Perturbação Møller-Plesset . . . . . . . . . . . . . . 34

2.5 Teoria do Funcional da Densidade . . . . . . . . . . . . . 38

2.5.1 Equações de Kohn-Sham . . . . . . . . . . . . . . . . 40

2.5 .2 DFT Dependente do Tempo (TD-DFT) . . . . . . . . . . . 44

$2.6 \quad$ Ajuste das Cargas Parciais - Método Chelpg . . . . . . . . . . . . . . 47

2.7 Modelo Contínuo para o Solvente . . . . . . . . . . . . . 51 
3 Mecânica Molecular $\quad 55$

3.1 Introdução . . . . . . . . . . . . . . . . . . 55

3.2 Método Monte Carlo . . . . . . . . . . . . . . . . 58

3.2 .1 Detalhes da Implementação . . . . . . . . . . . . . . . . 64

3.2 .2 Potencial de Interação Intermolecular . . . . . . . . . . . . . . . 66

3.2.3 Propriedades Estruturais: Função de Distribuição Radial . . . . 69

3.2 .4 Correlação Estatística com Função de Autocorrelação . . . . . . 70

3.2 .5 Energia Livre com Perturbação Termodinâmica . . . . . . . . . 72

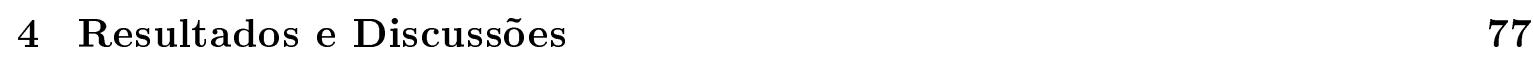

4.1 Estudo da Molécula Isolada . . . . . . . . . . . . . . . . . . . 77

$4.1 .1 \quad$ Análise Conformacional . . . . . . . . . . . . . . . 78

4.1 .2 Espectro de Absorção . . . . . . . . . . . . . . . . . . . 89

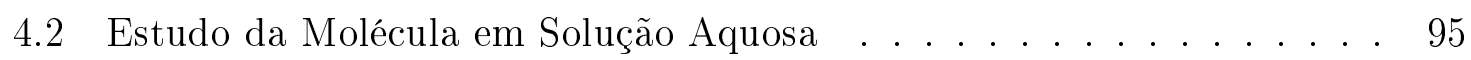

$4.2 .1 \quad$ Detalhes da Simulação . . . . . . . . . . . . . . . 96

4.2 .2 Ligação de Hidrogênio . . . . . . . . . . . . . . . . . 102

4.2 .3 Camadas de Solvatação. . . . . . . . . . . . . . . . . . . 104

4.2 .4 Incluindo a Polarização do OM em Água . . . . . . . . . . . . . 106

4.2 .5 Ligação de Hidrogênio: Soluto Polarizado . . . . . . . . . . . . . 111

4.2 .6 Camadas de Solvatação: Soluto Polarizado . . . . . . . . . . . 113

4.2 .7 Análise Conformacional . . . . . . . . . . . . . . 114

4.2 .8 Espectro de Absorção . . . . . . . . . . . . . . . . . 122

$\begin{array}{lll}5 & \text { Sumário e Conclusões } & 127\end{array}$

$\begin{array}{ll}\text { Referências Bibliográficas } & 131\end{array}$

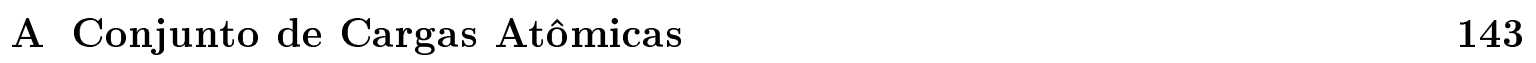

\begin{tabular}{lll}
\hline B Parâmetros Usados na Simulação. & 147
\end{tabular} 


\section{Lista de Abreviaturas}

B3LYP Becke's three-parameter exchange + Lee-Yang-Parr correlation

BHandHLYP Becke's half-and-half exchange + Lee-Yang-Parr correlation

CHELPG Charges from Electrostatic Potential Grid based

DFT Teoria do Funcional da Densidade

GGA Aproximação do Gradiente Generalizado

HF Hartree-Fock

HK Hohemberg e Kohn

HOMO Orbital Molecular Mais Alto Ocupado

HUMO Orbital Molecular Mais Baixo Desocupado

IV Infravermelho

KS Konh-Sham

LCAO Combinação Linear de Orbitais Atômicos

LDA Aproximação de Densidade Local

LH Ligações de Hidrogênio

LSDA Aproximação de Densidade de Spin-Local

MC Monte Carlo

MDDF RDF da Mínima Distância entre os átomos do soluto e os átomos do solvente

MM Mecânica Molecular

MP Teoria de Perturbação Møller Plesset

MP2 Teoria de Perturbação de Møller Plesset de segunda ordem 


$\begin{array}{ll}\text { OM } & \text { Óxido Mesitil } \\ \text { OIM } & \text { Óxido Isomesitil } \\ \text { PBE } & \text { Perdew-Burke-Erzenhof } \\ \text { PCM } & \text { Modelo Contínuo Polarizável } \\ \text { QM } & \text { Mecânica Quântica } \\ \text { RDF } & \text { Função de Distribuição Radial } \\ \text { SCF } & \text { Campo Auto-Consistente } \\ \text { S-QM/MM } & \text { Sequêncial- Mecânica Quântica/Mecânica Molecular } \\ \text { TD-DFT } & \text { Teoria do Funcional da Densidade Dependente do Tempo } \\ \text { TPRS } & \text { Teoria de Perturbação Rayleigh-Schrödinger } \\ \text { TPT } & \text { Teoria de Perturbação Termodinâmica } \\ \text { TS } & \text { Estado de Transição }\end{array}$




\section{Lista de Figuras}

$1.1 \quad$ Ilustração dos isômeros syn e anti da molécula OM. . . . . . . . . . . 2

1.2 Ilustração dos isômeros syn e anti da molécula OIM. . . . . . . . . . . . 3

1.3 Ilustração da forma enol para a molécula OM. . . . . . . . . . . . . 4

1.4 Comportamento da energia de transição do OM para diferentes solventes segundo a escala $Z$ de polaridade. Os solventes são (1) água, (2)

2,2,3,3-tetrafluoro-1-propanol, (3) metanol, (4) etilenoglicol, (5) etanol (95\%), (6) etanol, (7) 1-butanol, (8) 2-propanol, (9) acetonitrila, (10) iso-octano, (11) clorofórmio. Figura retirada da referência $|9|$. . . . . 5

1.5 Comportamento da energia de transição do OM, $E_{T}$, para diferentes solventes em duas escalas de polaridade. Os solventes são (1) água, (2) 2,2,3,3-tetrafluoro-1-propanol, (3) metanol, (4) etilenoglicol, (5) etanol (95\%), (6) etanol, (7) 1-butanol, (8) 2-propanol, (9) acetonitrila, (10) iso-octano, (11) clorofórmio. (b) Figura retirada da referência $|9|$. . . . 10

3.1 Ilustração da interação entre os sítios $i$ da molécula $a$ e os sítios $j$ da molécula $b$. Figura retirada de $[88] \ldots \ldots \ldots$. . . . . . . . 68

3.2 Ilustração da estrutura de um líquido atômico e a representação dos picos da $G(r)$ correspondente. Isômero anti da molécula OM. . . . . . . 70 
3.3 Típica função de autocorrelação da energia, calculada para o caso do isômero syn do Óxido Mesitil em água, usando método Monte Carlo. Ensemble NPT condições normais de pressão e temperatura . . . . . . 72

4.1 Ilustração da molécula de OM e numeração dos átomos. . . . . . . . . . 79

4.2 Ilustração dos isômeros do OM estudados, syn e anti, e o estado de transição, TS. . . . . . . . . . . . . . . . . . . 79

4.3 Ilustração dos orbitais envolvidos na transição mais intensa $\left(\pi-\pi^{*}\right)$ do OM isolado. . . . . . . . . . . . . . . . . . . . 990

4.4 Ilustração dos grupos funcionais da molécula OM. Isômero anti. . . . 99

4.5 Energia por molécula versus o número de ciclos durante o processo de termalização do sistema, isômero syn. . . . . . . . . . . . . . . . 101

4.6 Função de auto-correlação estatística da energia potencial de interação por molécula, $U / N$. Isômero syn. . . . . . . . . . . . . . . . 101

4.7 Função de distribuição radial de pares, $G_{O O}(\mathrm{r})$.

4.8 RDF de mínima distância entre o OM e as moléculas de água. . . . . . 106

4.9 Ilustração do processo iterativo para polarização do soluto. . . . . . . . 107

4.10 Evolução do momento de dipolo com o processo de polarização iterativo. Isômero syn. Linha pontilhada é o valor do dipolo obtido com o modelo contínuo de solvente PCM. . . . . . . . . . . . . . . . . . . . . . 110

4.11 Evolução do momento de dipolo com o processo de polarização iterativo.

\begin{tabular}{|c|}
\hline Isômero anti. Linha pontilhada é o valor do dipolo obtido com o modelo \\
\hline
\end{tabular}

4.12 Função de distribuição radial de pares, $G_{O O}(\mathrm{r})$. 
4.13 RDF de mínima distância entre o OM e as moléculas de água. Isômero

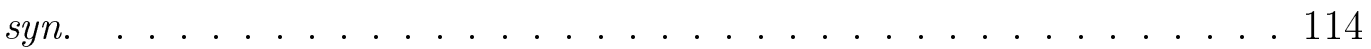

4.14 Ilustração do ciclo termodinâmico para o cálculo da variação de energia livre da mudança conformacional syn $\rightarrow$ anti em solução aquosa. . . . . 115

4.15 Deslocamento solvatocrômico versus número de águas explícitas. Isômero

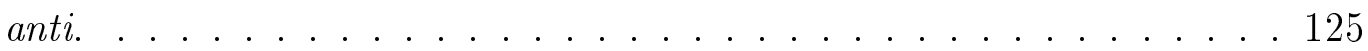




\section{Lista de Tabelas}

1.1 Resultados experimentais para o comprimento de onda, $\lambda_{\max }(\mathrm{nm})$, de absorção do OM em diferentes solventes e valores estimados para $Z$ (kcal/mol), que é uma escala empírica adotada para definir a polaridade do solvente. Dados retirados da referência $|9| \ldots \ldots$. . . . . . . . 6

4.1 Distância das ligações entre os átomos obtidas nos diferentes níveis de cálculo e funções base. . . . . . . . . . . . . . . . . . . . 80

4.2 Valores dos ângulos de ligação para as conformações obtidos nos diferentes níveis de cálculos QM. . . . . . . . . . . . . . . . . . . . . 82

4.3 Valores diedrais para as conformações e os respectivos métodos utilizados. 83

4.4 Tabela com as variações da energia (kcal $/ \mathrm{mol}$ ) calculada com diferentes níveis de cálculos QM. . . . . . . . . . . . . . . . . . . . . 85

4.5 Correções térmicas e energia de ponto zero relativas $(\mathrm{kcal} / \mathrm{mol})$ obtidas com diferentes níves de cálculos QM. . . . . . . . . . . . . . . . 86

4.6 Tabela com as variações da energia livre (kcal/mol) calculada com diferentes níveis de cálculos QM. . . . . . . . . . . . . . . . 86

4.7 Tabela com as variações da energia livre (kcal $/ \mathrm{mol}$ ) calculada com diferentes níveis de cálculos QM. . . . . . . . . . . . . . . . . . . 88 
4.8 Dipolo(D) do OM nas conformações syn, TS e anti em fase gasosa calculado usando diferentes níveis de cálculos QM. . . . . . . . . . . . . . 88

4.9 Energia de absorção $\left(\mathrm{cm}^{-1}\right)$ da transição $\pi-\pi^{*}$ do OM em função da base e o respectivo tempo de CPU envolvido no cálculo. . . . . . . . . . 91

4.10 Energia de absorção $\left(\mathrm{cm}^{-1}\right)$ da transição $\pi-\pi^{*}$ do OM em função da base. Funcional PBE. . . . . . . . . . . . . . . . . . . . . . 93

4.11 Energia de absorção $\left(\mathrm{cm}^{-1}\right)$ da transição $\pi-\pi^{*}$ do OM em função da base. Funcional BHandHLYP. . . . . . . . . . . . . . . . . . . . . . . 94

4.12 Análise do conjunto de cargas do OPLS para os dois isômeros. . . . . . 98

4.13 Análise da carga dos grupos funcionais da molécula OM. Valores obtidos com cálculos quânticos (Gás) e através do campo de força (OPLS) . . . 99

4.14 Análise estatística das ligações de hidrogênio. . . . . . . . . . . . . 105

4.15 Conjunto das cargas para o isômero anti. . . . . . . . . . . . 109

4.16 Análise estatística das ligações de hidrogênio. . . . . . . . . . . . . . 112

4.17 Tabela com as contribuições para a energia livre de solvatação em água (kcal/mol) obtidas considerando o solvente como um meio contínuo

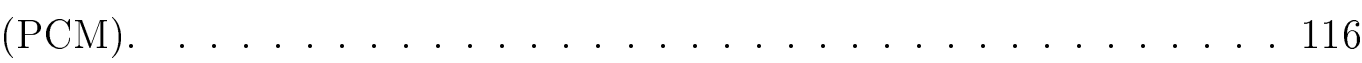

4.18 Tabela com a variação de energia livre (kcal/mol) necessária para desaperecer com a contribuição eletrostática do OM em água. . . . . . . . 118

4.19 Tabela com a variação de energia livre (kcal/mol) necessária para desaperecer com a contribuição de van der Waals do OM em água. ... 119

4.20 Tabela com a variação de energia livre (kcal $/ \mathrm{mol}$ ) necessária para desaparecer com o termo repulsivo, cavitação do OM em água. . . . . 119 
4.21 Valores da variação de energia livre $(\mathrm{kcal} / \mathrm{mol})$ obtida em cada estágio, a energia livre total para desaparecer com o OM $\left(\Delta G_{\times \rightarrow 0}\right)$ e a energia livre de solvatação aquosa do $\mathrm{OM}\left(\Delta G_{\times}\right)$nos diferentes isômeros. Apresentamos também as diferenças da energia livre associadas às mudanças conformações. . . . . . . . . . . . . . . . . . . 120

4.22 Tabela com os valores da variação de energia livre (kcal $/ \mathrm{mol})$ associada as mudanças syn $\rightarrow$ anti e syn $\rightarrow T S$, com os valores de fase gasosa obtidos pelos diferentes métodos. . . . . . . . . . . . . . 121

4.23 Efeito do solvente na energia de absorção $\left(\mathrm{cm}^{-1}\right)$ da transição $\pi-\pi^{*}$ do OM. Os valores foram obtidos com TD/DFT usando o conjunto de funções base $6-311++\mathrm{G}^{* *} . \Delta E_{\text {agua }}=E_{\text {água }}-E_{\text {gás }} \ldots \ldots \ldots$. . . . 123

4.24 Efeito do solvente no deslocamento da energia de absorção $\left(\mathrm{cm}^{-1}\right)$ da transição $\pi-\pi^{*}$ do OM. Os valores foram obtidos com TD/DFT usando o conjunto de funções base $6-311++\mathrm{G}^{* *}$. . . . . . . . . . . . 125

A.1 Teste do potencial eletrostático para o isômero syn. . . . . . . . . . . . 144

A.2 Teste do potencial eletrostático para o estado de transição $(T S)$. . . . . 145

B.1 Tabela com os parâmetros necessários para realizar uma simulação no DICE. Isômero syn f . . . . . . . . . . . . . . . . . 148

B.2 Tabela com os parâmetros necessários para realizar uma simulação no DICE. Estado de transição TS . . . . . . . . . . . . . . . . . . 149

B.3 Tabela com os parâmetros necessários para realizar uma simulação no DICE. Isômero anti. . . . . . . . . . . . . . . . . . . . . 150

B.4 Parâmetros obtidos do modelo SPC para a água |101|. . . . . . . . . 151 


\section{Capítulo 1}

\section{Introdução}

Nos últimos anos, tem havido uma grande necessidade em compreender melhor o efeito do solvente em propriedades moleculares e também em processos físico-químicos, pois é em solução que ocorre grande parte das reações químicas [1,2], que são de fundamental importância em processos biológicos e em aplicações tecnológicas. Além da importância prática de líquidos como meio para muitas reações, o papel do solvente como mediador de vários processos constitui uma área complexa e de importante interesse científico. Os efeitos do solvente vêm sendo muito estudados a mais de um século, pois qualquer medida que seja realizada em solução, desde velocidades de reações à posição

e intensidade de bandas espectrais, é influenciado pelo meio [3].

O solvente pode modificar as propriedades de um soluto de várias formas. Ele pode, em particular, afetar a estabilidade conformacional de moléculas [4,5]. Isso acontece porque, em geral, a diferença de energia livre entre as diferentes conformações é pequena, portanto, em um dado solvente haverá uma maior população de uma conformação e em outro não. O que se tem como senso comum é que a conformação com maior momento de dipolo deve ser mais estável em solventes com uma constante dielétrica $(\epsilon)$ alta $[6]$.

Nesse sentido, realizamos estudos teóricos para investigarmos o efeito do solvente na estabilidade conformacional da molécula Óxido Mesitil $\left(\left(\mathrm{CH}_{3}\right)_{2} \mathrm{C}=\mathrm{CHCOCH}_{3}\right)$. 
Essa molécula, por ser pequena, é factível de estudos teóricos de alto nível de cálculos quânticos, e pode ser considerada como um modelo para compostos maiores que contém o grupo cetona. Esses compostos são caracterizados pela presença do grupamento $\mathrm{C}=\mathrm{O}$ (carbonila) ligado a dois outros grupos orgânicos e apresentam uma fórmula geral $\mathrm{RC}-\mathrm{C}(=\mathrm{O})-\mathrm{CR}$ '. Sabe-se que a transição eletrônica de baixo comprimento de onda de compostos $\alpha, \beta$-cetonas insaturadas são muito sensíveis ao solvente, daí o interesse no estudo dessa molécula.

A molécula Óxido Mesitil (OM) apresenta 5 isômeros. Na forma cetona conjugada, em que há a presença do grupamento $-\mathrm{C}=\mathrm{O}$ numa cadeia conjugada de carbonos $\mathrm{C}-\mathrm{C}=\mathrm{C}-$, essa molécula pode ser encontrada nas formas syn e anti, figura (1.1).

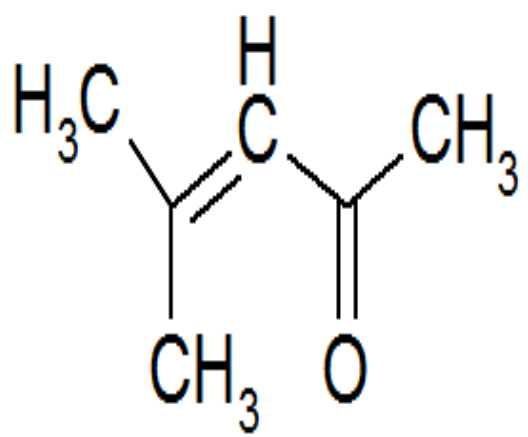

(a) syn

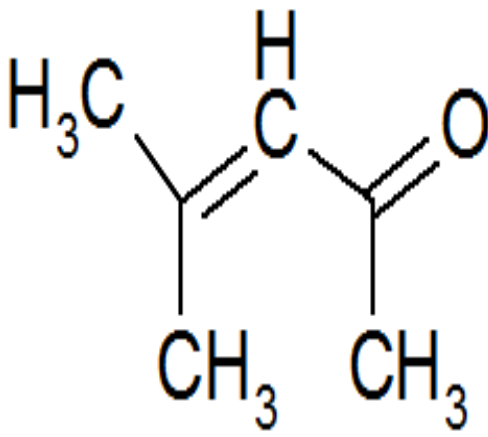

(b) anti

Figura 1.1: Ilustração dos isômeros syn e anti da molécula OM.

Esses isômeros diferem pela orientação relativa do oxigênio com a cadeia conjugada através do ângulo torcional entre os átomos $\mathrm{C}=\mathrm{C}-\mathrm{C}=\mathrm{O}$. Na conformação syn (antigamente conhecida como cis) esse ângulo é de $0^{\circ}$ e na conformação anti (antigamente conhecida como trans) é $180^{\circ}$. Na forma cetona não conjugada, esse isômero é chamado de Óxido Isomesitil (OIM, $\left.\mathrm{CH}_{2}=\mathrm{C}-\left(\mathrm{CH}_{3}\right) \mathrm{CH}_{2} \mathrm{COCH}_{3}\right)$ e também pode ser encontrado nas formas syn e anti, figura (1.2). Experimentalmente foi observado 7] que mesmo após o isolamento e purificação do $\mathrm{OM}$, ainda se encontra uma população 
de $9 \%$ do OIM, nas condições normais de temperatura e pressão.

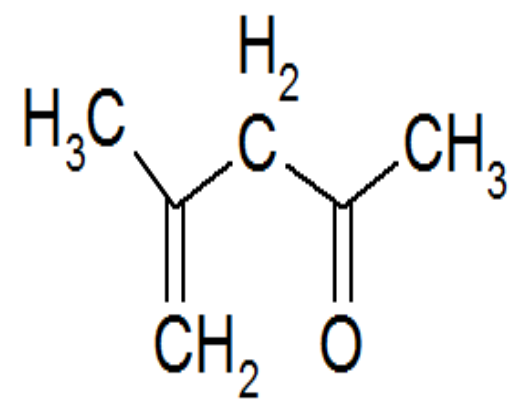

(a) syn

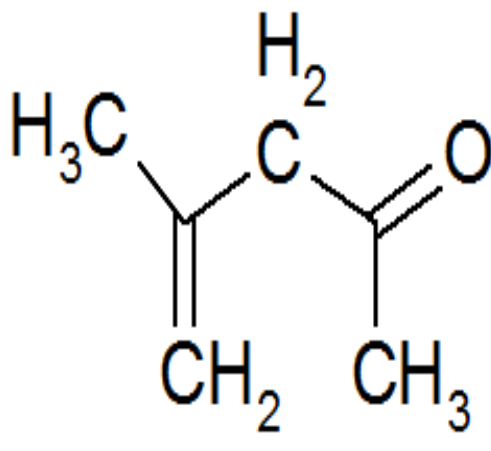

(b) anti

Figura 1.2: Ilustração dos isômeros syn e anti da molécula OIM.

Uma outra possibilidade de conformação para compostos que possuam pelo menos um hidrogênio $\alpha$, hidrogênio ligado ao carbono imediatamente adjacente ao carbonila, é a forma enólica, figura (1.3). No entanto, na maioria dos casos a forma cetona $(\mathrm{RC}-\mathrm{C}(=\mathrm{O})-\mathrm{CR}$ ') é termodinâmicamente mais estável do que a foma enólica, pois a ligação $\pi$ carbono-oxigênio $(\mathrm{C}=\mathrm{O})$ é mais forte do que a ligação $\pi$ carbonocarbono $(\mathrm{C}=\mathrm{C})$. Para o $\mathrm{OM}$ foi verificado experimentalmente [8], usando espectro vibracional, que o isômero na forma enólica não é encontrado na condições normais de temperatura e pressão.

Experimentalmente, os dois isômeros OM e OIM foram caracterizados através do espectro vibracional (região do infravermelho,IV), do ponto de ebulição e do espectro eletrônico de absorção (UV-vis) [7,8]. O OM apresenta um ponto de ebulição mais alto (em $130{ }^{\circ} \mathrm{C}$ ), uma banda intensa no IV que caracteriza a ligação $\mathrm{C}=\mathrm{O}$ numa posição conjugada e duas bandas no UV-vis (231 e $329 \mathrm{~nm}$ em iso-octano). Essas bandas também são similares, em posição e intensidade, a outros compostos que apresentam o grupo cetona em cadeias conjugadas. O OIM apresenta ponto de ebulição mais baixo $\left(121.5{ }^{\circ} \mathrm{C}\right)$, uma banda no IV que caracteriza a ligação $\mathrm{C}=\mathrm{O}$ numa posição 


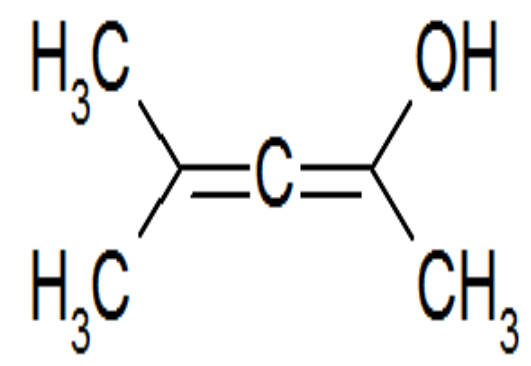

Figura 1.3: Ilustração da forma enol para a molécula OM.

não conjugada e uma banda no UV-vis (em 290 nm em iso-octano) característica de compostos cetona não conjugados. Observa-se que há uma proximidade da banda de menor comprimento de onda desses isômeros em 290 e 329 nm (34500 e 30400 $\left.\mathrm{cm}^{-1}\right)$. Portanto, é sugerido que apenas a banda em $231 \mathrm{~nm}\left(43300 \mathrm{~cm}^{-1}\right)$ seja usada para a caracterização do OM, pois devido a composição mista no equilíbrio (91\% de OM e $9 \%$ de OIM) os resultados experimentais para a banda na região de 290-330 nm não se refere puramente ao composto OM pois o isômero OIM também absorve nessa região. Dada as dificuldades no tratamento teórico dessa composição mista, aqui iremos descrever o efeito do solvente apenas na banda da região de $231 \mathrm{~nm}$ onde há absorção apenas do OM. Portanto, neste trabalho foram feitas investigações teóricas apenas da estabilidade conformacional dos isômeros syn e anti da forma cetona conjugada do OM.

\subsection{Polaridade de Solventes}

Em 1958, Kosower [9] estudou, experimentalmente, os efeitos de solventes no espectro eletrônico de absorção do OM e analizou os deslocamentos das bandas utilizando uma escala de polaridade empírica $Z[10]$. Na tabela (1.1) são apresentados esses resultados numéricos e na figura (1.4) a relação entre a energia de transição 
$\left(E_{T}=28591 / \lambda_{\max }\right)^{1}$ e a polaridade do solvente, $Z$.

A escala $Z$ de Kosower é uma medida da polaridade do solvente e foi montada a partir dos máximos de absorção $\left(\lambda_{\max }\right)$ da banda de transferência de carga para o iodeto de 1-etil-4-metoxicarbonilpiridínio. Seguindo essa escala o solvente menos polar é o iso-octano, $Z=60.1 \mathrm{kcal} / \mathrm{mol}$, e o mais polar é a água com $Z=94.6 \mathrm{kcal} / \mathrm{mol}$.

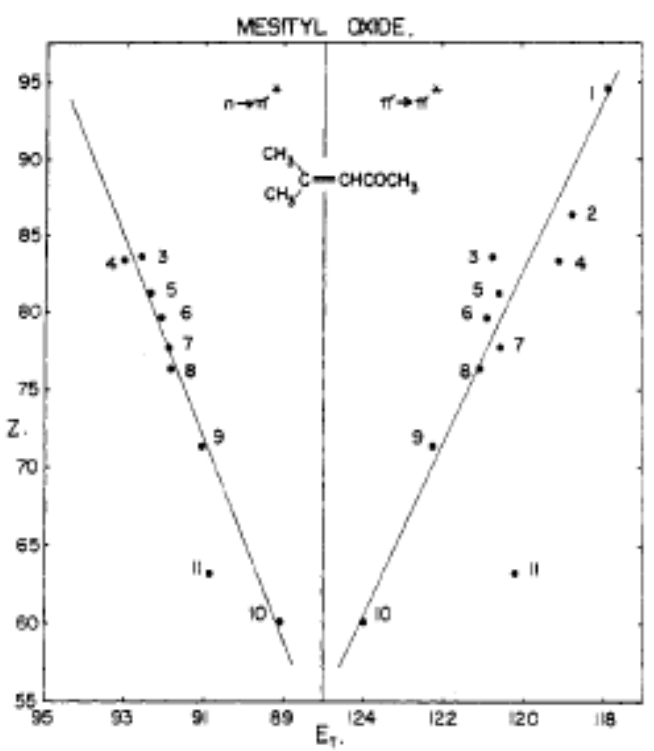

Figura 1.4: Comportamento da energia de transição do OM para diferentes solventes segundo a escala $Z$ de polaridade. Os solventes são (1) água, (2) 2,2,3,3-tetrafluoro-1-propanol, (3) metanol, (4) etilenoglicol, (5) etanol (95\%), (6) etanol, (7) 1-butanol, (8) 2-propanol, (9) acetonitrila, (10) iso-octano, (11) clorofórmio. Figura retirada da referência [9].

Na literatura é sabido que o espectro eletrônico de absorção de um soluto medido em solventes de diferentes polaridades pode apresentar mudanças na posição, intensidade e na forma das bandas de absorção [11, 12]. O termo solvatocromismo é usado para descrever as mudanças na posição dos máximos, $\lambda_{\text {max }}$, das bandas devido a mudança na polaridade do meio. Um deslocamento do $\lambda_{\max }$ para menores comprimentos de onda (ou maiores energias) com o aumento da polaridade do solvente é conhecido como deslocamento hipsocrômico (ou deslocamento para o azul) e caracteriza um sol-

\footnotetext{
${ }^{1}$ Essa expressão será discutida a seguir, equação 1.2
} 
Tabela 1.1: Resultados experimentais para o comprimento de onda, $\lambda_{\max }(\mathrm{nm})$, de absorção do $\mathrm{OM}$ em diferentes solventes e valores estimados para $Z(\mathrm{kcal} / \mathrm{mol})$, que é uma escala empírica adotada para definir a polaridade do solvente. Dados retirados da referência [9]

\begin{tabular}{|c|c|c|c|}
\hline Solvente & $\begin{array}{c}1^{\mathrm{a}} \text { transição }\left(\lambda_{\max }\right) \\
n-\pi^{*}\end{array}$ & $\begin{array}{c}2^{\mathrm{a}} \text { transição }\left(\lambda_{\max }\right) \\
\pi-\pi^{*}\end{array}$ & $Z$ \\
\hline \multirow[t]{2}{*}{ Iso-octano } & 321 & 231 & 60.1 \\
\hline & $329^{a}$ & $231^{a}$ & \\
\hline 1,2-Dicloroetano & 318 & 236 & $\cdots$ \\
\hline Clorofórmio, puro & 315 & 238 & $\cdots$ \\
\hline Clorofórmio (Etanol $0.13 \mathrm{M}$ ) & 315 & 238 & $\sim 64$ \\
\hline Acetonitrila & 314 & 234 & $\sim 72$ \\
\hline Álcool isopropil & 311 & 236 & $\sim 76$ \\
\hline Álcool n-butil & 311 & 237 & $\sim 77$ \\
\hline Etanol & 311 & 236 & $\sim 79$ \\
\hline Etanol $(95 \%)$ & 310 & 237 & $\sim 81$ \\
\hline Etilenoglicol & 307 & 240 & $\sim 83$ \\
\hline Metanol & 309 & 237 & $\sim 84$ \\
\hline Tetrafluopropanol & $\cdots$ & 241 & $\sim 87$ \\
\hline Dimetilsulfóxido & 320 & $\cdots$ & $\cdots$ \\
\hline Água & $\ldots$ & 243 & 94.6 \\
\hline
\end{tabular}

${ }^{a}$ ref. $|8|$ 
vatocromismo negativo. Já um deslocamento do $\lambda_{\max }$ para maiores comprimentos de onda (ou menores energias) é conhecido como deslocamento batocrômico (ou deslocamento para o vermelho) e caracteriza um solvatocromismo positivo.

O solvatocromismo é causado pela solvatação diferencial do estado fundamental e do estado excitado da molécula (ou seu cromóforo) que absorve a luz e pode ser explicado pela diferença que existe na interação soluto-solvente nesses dois estados. Assim, se com o aumento da polaridade o estado fundamental da molécula for melhor estabilizado pela solvatação do que o estado excitado (a energia do estado fundamental diminuirá mais do que a energia do estado excitado) a transição envolvendo os dois estados será deslocada para o azul, caracterizando um solvatocromismo negativo. Por outro lado, se o estado excitado envolvido na transição for melhor estabilizado pela solvatação com o aumento da polaridade (energia do estado excitado diminuirá mais do que a energia do estado fundamental) a transição entre os dois estados será deslocada para o vermelho, caracterizando um solvatocromismo positivo. Aqui o primeiro estado excitado mencionado é chamado de estado excitado de Franck-Condon [13, 14 e possui a mesma geometria do estado fundamental, pois o tempo necessário para um molécula ser excitada eletrônicamente, algo em torno de $10^{-15}$ s, é muito menor que o tempo de relaxação da geometria.

Na descrição do solvatocromismo, as propriedades físicas e químicas do solvente são muito importantes para uma completa compreensão à respeito da interação solutosolvente, ou seja, qual a influência no soluto devido a presença de um dado solvente. Essa influência é discutida no que diz respeito à polaridade do solvente. Aparentemente, a definição de polaridade do solvente não pode ser descrita quantitativamente por um único parâmetro físico do solvente. Há modelos que buscam a descrição da solvatação de moléculas dipolares considerando o solvente como uma estrutura contínua, em que é levado em conta apenas parâmetros macroscópicos do solvente, como a constante dielétrica $(\epsilon)$, o momento de dipolo $(\mu)$ e o índice de refração. Entretanto, 
as interações soluto-solvente ocorrem em um nível molecular microscópico, dentro de uma estrutura descontínua consistindo de moléculas individuais do solvente, capazes de interações mútuas soluto-solvente. Desta forma, somente essas grandezas físicas, que dão conta apenas das interações intermoleculares não específicas, são inadequadas para uma medida quantitativa da polaridade do solvente. Uma definição favorável de polaridade do solvente é a capacidade total de solvatação (ou força de solvatação) dos solventes, a qual inclui todas as interações intermoleculares específicas e não específicas. Portanto, a polaridade é a soma de todas propriedades moleculares responsáveis por todas as forças de interação (Coulomb, direcional, indutiva, dispersão, ligações de hidrogênio e forças de interação envolvendo doador e receptor de pares de elétrons) [15].

A polaridade de solventes tem sido comumente estudada através da energia de transição $\left(E_{T}\right)$ de corantes solvatocrômicos, esses corantes são moléculas que apresentam bandas de absorção na região visível do espectro e sofrem deslocamento à medida que a polaridade do meio se altera [16]. Portanto, pode-se obter parâmetros empíricos de polaridades dos solventes através de medidas de UV/vis. Com isso, o espectro de absorção de uma grande variedade de compostos, que apresenta dependência com a polaridade do solvente, tem sido estudado como forma de encontrar uma possível referência para uma escala empírica de polaridade fazendo com que inúmeras escalas de polaridades do solvente fossem publicadas [1, 10, 17, 18.

Nesse sentido, Reichardt [1] elaborou uma escala empírica uniparamétrica de polaridade de solventes que recebeu a denominação de $E_{T}(30)$, a qual se baseia no forte solvatocromismo exibido pelo corante 2,6-difenil-4-(2,4,6-trifenil-1-piridínio)-1fenolato, conhecida como betaína 30 de Reichardt (B30), e é definida pela energia de transição (kcal/mol) desse corante. Essa energia é obtida através da seguinte equação 


$$
\begin{aligned}
E_{T}(30)(\mathrm{kcal} / \mathrm{mol}) & =h c \tilde{\nu}_{\max } N_{A} \\
& =28591 / \lambda_{\max }(\mathrm{nm})
\end{aligned}
$$

onde $E_{T}(30)$ é a energia de transição molar do composto em um determinado solvente, $h$ é a constante de Planck, $c$ é a velocidade da luz no vácuo, $\tilde{\nu}_{\text {max }}$ é o número de onda de máxima absorção, $N_{A}$ é o número de Avogadro e $\lambda_{\max }$ é o comprimento de onda de absorção máxima. Foram determinados valores para a energia $E_{T}(30)$ em mais de 360 solventes orgânicos puros [1,2] e para um grande número de misturas binárias [2]. Com base nesses valores os solventes foram ordenados por ordem de polaridade, onde um valor pequeno de $E_{T}(30)$ indica que a energia da transição da B30 foi medida num solvente de baixa polaridade e um valor grande de $E_{T}(30)$ indica que a medida foi feita num solvente de alta polaridade. Seguindo essa escala o solvente menos polar é o tetrametilsilano (TMS) com $E_{T}(30)=30.7 \mathrm{kcal} / \mathrm{mol}$ e o mais polar é a água com $E_{T}(30)=63.1 \mathrm{kcal} / \mathrm{mol}$. Nessa escala, o solvente iso-octano apresenta o valor de 31.0 $\mathrm{kcal} / \mathrm{mol}$, e está entre os solventes de mais baixa polaridade

Através da escala de polaridade da B30 também podemos observar como se dá a interação soluto-solvente na molécula OM. Usando os dados experimentais para a energia de absorção da molécula OM em diferentes solventes, tabela (1.1), podemos obter o comportamento do seu deslocamento solvatocrômico, para solventes de diferentes polaridades, relacionando com o deslocamento apresentado pela B30. Na figura (1.5), está mostrado o deslocamento da energia de transição da banda $\pi-\pi^{*}$ do OM nos diversos solventes, $E_{T}$, em relação aos valores de $E_{T}(30)$ para os mesmos solventes, comparativamente ao deslocamento verificado pela escala $Z$ de Kosower.

Na figura 1.5 observa-se na escala $\mathrm{E}_{T}(30)$ que com o aumento da polaridade do solvente há uma diminuição da energia de transição do OM, ou seja, um deslocamento batocrômico ou deslocamento para o vermelho (solvatocromismo positivo), o que, nor- 


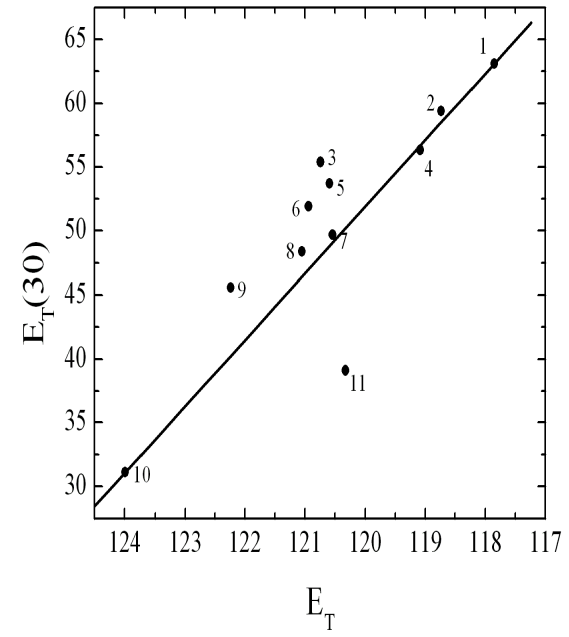

(a) Escala $E_{T}(30)$

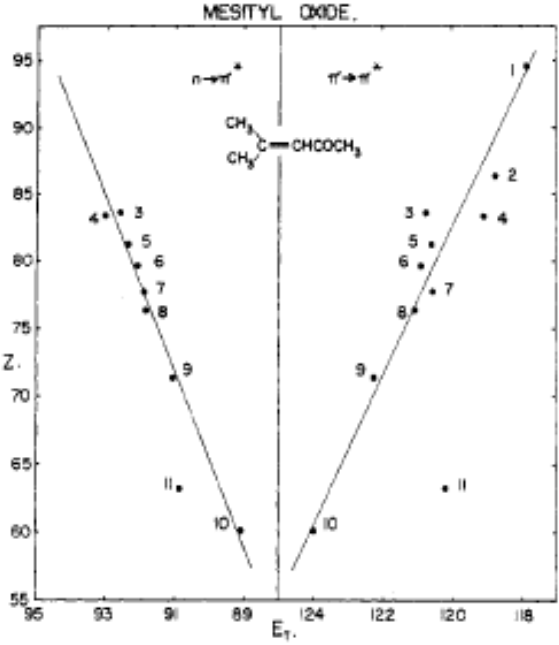

(b) Escala $Z$ de Kosower

Figura 1.5: Comportamento da energia de transição do $\mathrm{OM}, E_{T}$, para diferentes solventes em duas escalas de polaridade. Os solventes são (1) água, (2) 2,2,3,3-tetrafluoro-1-propanol, (3) metanol, (4) etilenoglicol, (5) etanol (95\%), (6) etanol, (7) 1-butanol, (8) 2-propanol, (9) acetonitrila, (10) iso-octano, (11) clorofórmio. (b) Figura retirada da referência [9].

malmente, caracteriza uma transição $\pi-\pi^{*}$. Observa-se também que as duas escalas apresentaram o mesmo comportamento. É interessante que na escala de polaridade do solvente, normalmente, são encontradas 2 regressões lineares, uma para os solventes que formam ligações de hidrogênio com o soluto (solventes próticos) e outra para os que não formam essas ligações (solventes apróticos) [1,9]. Portanto, seria de se esperar que pelo menos o ponto 1 (solvente água) dos gráficos da figura (1.5), estivessem fora da regressão linear. O fato desse ponto está perfeitamente alinhado na regressão é intrigante e levanta a possibilidade de que o solvente está provocando algum tipo de efeito adicional ou de deformação da nuvem eletrônica que é observado para o B30. Uma vez que evidências experimentais descartam possibilidade de mudanças conformacionais e agregações do B30 [1,2]. 


\subsection{Modelos para o Solvente}

Como foi dito anteriormente a interação com o solvente pode mudar as propriedades das moléculas, como exemplo, deslocamentos das bandas de absorção eletrônica, velocidades das reações, entre outras. Portanto, é necessário uma descrição adequada do solvente para que se possa reproduzir tais efeitos. Neste sentido, há dois modelos que tentam mimetizar o meio: modelos contínuos para o solvente e modelos discretos.

O modelo contínuo é uma aproximação que considera o solvente de maneira implícita, sendo esse baseado numa descrição contínua do meio [19,20. Nesse caso, as moléculas de interesse são colocadas em uma cavidade num meio contínuo polarizável e a única informação necessária do solvente é a constante dielétrica, $\epsilon$. Nessa aproximação os efeitos do solvente são expressos através de um campo elétrico médio provocado por esse na molécula de interesse. Um dos pontos a favor do modelo contínuo é a possibilidade de se fazer um tratamento puramente quântico da interação soluto-solvente, uma vez que esses modelos tratam o solvente sem aumentar o número de elétrons explícitos. Além do mais, esse modelo apresenta um baixo custo computacional. Porém, como desvantagens surgem a perda de todas as interações específicas do solvente com as moléculas de interesse, moléculas apolares não podem ser tratadas e o fato de que propriedades moleculares podem depender do tamanho e da forma da cavidade.

Nos modelos discretos o solvente é tratado explicitamente. O que implica em lidar com um número maior de informações, uma vez que há a necessidade de se saber as posições de todas as moléculas do sistema. Nesse tratamento também torna-se necessário levar em conta a natureza estatística do líquido.

Há diferentes métodos que tratam o solvente explicitamente. Dentre eles há os que tratam o sistema(soluto + solvente) através de um hamiltoniano puramente quântico, tais como o da Dinâmica de Car Parrinelo (CPMD) 21] e o da Dinâmica 
Molecular Born-Oppenheimer (BOMD) [22]. Entretanto, a aplicação destes só é factível para sistemas pequenos, considerando poucas moléculas de solvente e também para simulações relativamente curtas. Por outro lado há as simulações clássicas, onde o solvente também é tratado explicitamente. Nessas simulações a interação intermolecular é modelada através de campos de força empíricos. Porém, as simulações clássicas estão limitadas a reproduzir apenas um conjunto de fenômenos, nos quais não há quebra de ligações químicas nem transferência de carga intra ou intermolecular. Um ponto a favor da utilização dessa aproximação clássica é que podemos calcular várias propriedades termodinâmicas e configuracionais e ainda, podemos gerar as configurações que constituem um líquido molecular.

Da necessidade de tratar o sistema quanticamente para visualizar processos reativos ou calcular propriedades eletrônicas, em sistemas maiores, surgiram os métodos chamados híbridos que combinam mecânica quântica (QM) e mecânica clássica ou mecânica molecular (MM). Esses métodos são chamados abreviadamente de QM/MM [23], onde o tratamento quântico restringe-se apenas à região envolvida no processo em estudo e o ambiente em que o subsistema está imerso é tratado classicamente como uma perturbação. No QM/MM os tratamentos quântico e clássico se dão ao mesmo tempo e, por isso, inúmeros cálculos quânticos são necessários ao longo de uma simulação. Isto acarreta num custo computacional elevado.

Como alternativa surgiu a metodologia $\mathrm{QM} / \mathrm{MM}$ sequencial, que trata as duas etapas separadas. Ao invés de tratar as partes clássica (MM) e quântica (QM) simultaneamente, ela faz de forma separada e sequencial; primeiro todo o sistema é tratado classicamente com MM e posteriormente quanticamente com QM. Essa metodologia foi proposta [24,25] como uma forma de diminuir algumas dificuldades do QM/MM convencional e, ao mesmo tempo, criar uma metodologia que garantisse que os resultados finais obtidos fossem médias estatisticamente convergidas. Além de ser menos custoso computacionalmente, comparado ao QM/MM convencional, a metodologia se- 
quencial também permite uma maior flexibilidade na escolha do sistema a ser tratado quanticamente. Uma outra vantagem dessa metodologia é que uma vez geradas as configurações, pode-se utilizá-las para realizar cálculos de várias propriedades.

Usando a metodologia QM/MM sequêncial nós investigamos o efeito do solvente no deslocamento solvatocrômico da molécula de Óxido Mesitil. Este trabalho está estruturado em 5 capítulos. Os capítulos 2 e 3 são referentes a descrição da metodologia teórica utilizada. No capítulo 2 descrevemos os métodos quânticos e no capítulo 3 descrevemos as simulações computacionais com mecânica molecular usando Monte Carlo.

No capítulo 4 apresentamos os resultados obtidos para a molécula OM isolada e em solução aquosa. No capítulo 5 apresentamos as conclusões e perspectivas deste trabalho. 


\section{Capítulo 2}

\section{Métodos Quânticos}

\subsection{Introdução}

Neste capítulo descrevemos os métodos de estrutura eletrônica que foram utilizados na realização deste trabalho. Abordamos de forma sucinta o método Hartree-Fock e métodos perturbativos pós Hartree-Fock. Falaremos também sobre a Teoria do Funcional da Densidade.

O objetivo central, utilizando métodos quânticos, é a obtenção de soluções da equação de Schrödinger para a determinação de propriedades de sistemas atômicos e moleculares. Porém, esse é um problema extremamente complicado e os diferentes métodos para a descrição da estrutura eletrônica de átomos e moléculas refletem as várias propostas de resolução dessa equação.

A equação de Schrödinger independente do tempo para uma molécula composta por N elétrons e M núcleos é escrita como:

$$
\mathcal{H}(\mathbf{r}, \mathbf{R}) \Psi(\mathbf{r}, \mathbf{R})=E \Psi(\mathbf{r}, \mathbf{R})
$$

onde $\mathcal{H}(\mathbf{r}, \mathbf{R})$ é o operador hamiltoniano, $\Psi(\mathbf{r}, \mathbf{R})$ é a função de onda total do sistema, $\mathbf{r}=\left(\mathbf{r}_{1}, \mathbf{r}_{2}, \ldots, \mathbf{r}_{\mathbf{N}}\right)$ e $\mathbf{R}=\left(\mathbf{R}_{1}, \mathbf{R}_{2}, \ldots, \mathbf{R}_{\mathbf{M}}\right)$ são as coordenadas dos elétrons e dos 
núcleos, respectivamente [26]. O hamiltoniano que descreve um sistema com $N$ elétrons e $M$ núcleos é expresso, em unidades atômicas (Hartree), por:

$$
\mathcal{H}(\mathbf{r}, \mathbf{R})=-\sum_{i=1}^{N} \frac{1}{2} \nabla_{i}^{2}-\sum_{A=1}^{M} \frac{1}{2 M_{A}} \nabla_{A}^{2}-\sum_{i=1}^{N} \sum_{A=1}^{M} \frac{Z_{A}}{r_{i A}}+\sum_{i<j}^{N} \frac{1}{r_{i j}}+\sum_{A<B}^{M} \frac{Z_{A} Z_{B}}{R_{A B}}
$$

onde os índices i e j referem-se aos elétrons e $\mathrm{A}$ e $\mathrm{B}$ aos núcleos, $\mathrm{Z}_{A}$ é a carga do núcleo $\mathrm{A}, \mathrm{M}_{A}$ é a massa do núcleo $\mathrm{A}$.

O primeiro passo para resolver a equação 2.1 é a separação dos movimentos nuclear e eletrônico. Tal aproximação é conhecida como aproximação de BornOppenheimer.

\subsection{Aproximação de Born-Oppenheimer}

A Aproximação de Born-Oppenheimer [27, 28] é fundamentada na diferença de escala energética que existe entre o núcleo atômico e o elétron. Sendo assim o hamiltoniano da equação $\left(2.2\right.$ pode ser separado em uma parte eletrônica $\left(\mathcal{H}^{\text {ele }}(\mathbf{r} ; \mathbf{R})\right)$ e outra nuclear $\left(\mathcal{H}^{N}(\mathbf{R})\right)$,

$$
\mathcal{H}(\mathbf{r}, \mathbf{R})=\mathcal{H}^{\text {ele }}(\mathbf{r} ; \mathbf{R})+\mathcal{H}^{N}(\mathbf{R})
$$

onde:

$$
\begin{gathered}
\mathcal{H}^{\text {ele }}(\mathbf{r} ; \mathbf{R})=-\sum_{i=1}^{N} \frac{1}{2} \nabla_{i}^{2}-\sum_{i=1}^{N} \sum_{A=1}^{M} \frac{Z_{A}}{r_{i A}}+\sum_{i<j}^{N} \frac{1}{r_{i j}} \\
\mathcal{H}^{N}(\mathbf{R})=-\sum_{A=1}^{M} \frac{1}{2 M_{A}} \nabla_{A}^{2}+\sum_{A<B}^{M} \frac{Z_{A} Z_{B}}{R_{A B}}
\end{gathered}
$$

Da mesma forma que o hamiltoniano total é obtido pela soma da parte eletrônica com a parte nuclear, a função de onda $\Psi(\mathbf{r}, \mathbf{R})$, que é autofunção de $\mathcal{H}(\mathbf{r}, \mathbf{R})$, pode 
ser escrita como o produto das funções de onda da parte eletrônica $\left(\Psi^{\text {ele }}(\mathbf{r} ; \mathbf{R})\right)$ com as autofunções do hamiltoniano nuclear $\left(\Psi^{N}(\mathbf{R})\right)$, logo:

$$
\Psi(\mathbf{r}, \mathbf{R})=\Psi^{\text {ele }}(\mathbf{r} ; \mathbf{R}) \Psi^{N}(\mathbf{R})
$$

onde $\Psi^{\text {ele }}(\mathbf{r} ; \mathbf{R})$ depende apenas parametricamente de $\mathbf{R}$. Uma vez que $\mathcal{H}^{\text {ele }}(\mathbf{r} ; \mathbf{R})$ e $\mathbf{R}$ podem ser diagonalizados simultaneamente, os autovalores do hamiltoniano eletrônico podem ser determinados para valores fixos das posições nucleares $\mathbf{R}$.

Reescrevendo a equação (2.1), temos:

$$
\left(\mathcal{H}^{\text {ele }}(\mathbf{r} ; \mathbf{R})+\mathcal{H}^{N}(\mathbf{R})\right) \Psi^{\text {ele }}(\mathbf{r} ; \mathbf{R}) \Psi^{N}(\mathbf{R})=E \Psi^{\text {ele }}(\mathbf{r} ; \mathbf{R}) \Psi^{N}(\mathbf{R})
$$

$$
\mathcal{H}^{\text {ele }}(\mathbf{r} ; \mathbf{R}) \Psi^{\text {ele }}(\mathbf{r} ; \mathbf{R}) \Psi^{N}(\mathbf{R})+\mathcal{H}^{N}(\mathbf{R}) \Psi^{\text {ele }}(\mathbf{r} ; \mathbf{R}) \Psi^{N}(\mathbf{R})=E \Psi^{\text {ele }}(\mathbf{r} ; \mathbf{R}) \Psi^{N}(\mathbf{R})
$$

onde o operador eletrônico atua somente na função de onda eletrônica e o operador nuclear atua na função de onda nuclear. Dividindo a expressão 2.8 por $\Psi^{\text {ele }}(\mathbf{r} ; \mathbf{R}) \Psi^{N}(\mathbf{R})$, obtemos:

$$
\begin{aligned}
& \frac{1}{\Psi^{\text {ele }}(\mathbf{r} ; \mathbf{R})} \mathcal{H}^{\text {ele }}(\mathbf{r} ; \mathbf{R}) \Psi^{\text {ele }}(\mathbf{r} ; \mathbf{R})+\frac{1}{\Psi^{N}(\mathbf{R})} \mathcal{H}^{N}(\mathbf{R}) \Psi^{N}(\mathbf{R})=E \\
& \frac{1}{\Psi^{\text {ele }}(\mathbf{r} ; \mathbf{R})} \mathcal{H}^{\text {ele }}(\mathbf{r} ; \mathbf{R}) \Psi^{\text {ele }}(\mathbf{r} ; \mathbf{R})=E-\frac{1}{\Psi^{N}(\mathbf{R})} \mathcal{H}^{N}(\mathbf{R}) \Psi^{N}(\mathbf{R})
\end{aligned}
$$

O termo à direita na igualdade da equação 2.10 depende somente das coordenadas nucleares, identificando esse termo com $\epsilon(\mathbf{R})$ temos:

$$
\mathcal{H}^{\text {ele }}(\mathbf{r} ; \mathbf{R}) \Psi^{\text {ele }}(\mathbf{r} ; \mathbf{R})=\epsilon(\mathbf{R}) \Psi^{\text {ele }}(\mathbf{r} ; \mathbf{R})
$$


onde

$$
\epsilon(\mathbf{R})=E-\frac{1}{\Psi^{N}(\mathbf{R})} \mathcal{H}^{N}(\mathbf{R}) \Psi^{N}(\mathbf{R})
$$

$\log \mathrm{O}$

$$
\mathcal{H}^{N}(\mathbf{R}) \Psi^{N}(\mathbf{R})+\epsilon(\mathbf{R}) \Psi^{N}(\mathbf{R})=E \Psi^{N}(\mathbf{R})
$$

que é a equação para o movimento dos núcleos, onde E é o autovalor da equação (2.1).

Portanto, a aproximação de Born-Oppenheimer separa a equação (2.1) em duas equações, 2.11) e (2.13), onde 2.11 é a equação do movimento eletrônico e 2.13 é a equação para o movimento dos núcleos. Devido a separação desses movimentos podemos fixar os núcleos em uma dada posição e então calcular $\Psi^{e l e}(\mathbf{r} ; \mathbf{R})$ pela equação (2.11). Assim, usando a equação (2.13) podemos obter a energia total, E(R). Contudo, vale lembrar que existem situações de forte acoplamento entre o movimento eletrônico e o movimento nuclear, onde a aproximação de Born-Oppenheimer não é válida [29].

Observando que para $\mathbf{R}$ fixo o único termo não nulo da expressão (2.5) é o potencial de repulsão núcleo-núcleo e que $\mathcal{H}^{\text {ele }}$ atua somente na função de onda eletrônica, podemos reescrever a equação (2.8) da seguinte forma:

$$
\epsilon(\mathbf{R}) \Psi^{\text {ele }}(\mathbf{r} ; \mathbf{R}) \Psi^{N}(\mathbf{R})+\sum_{A<B}^{M} \frac{Z_{A} Z_{B}}{R_{A B}} \Psi^{\text {ele }}(\mathbf{r} ; \mathbf{R}) \Psi^{N}(\mathbf{R})=E(\mathbf{R}) \Psi^{\text {ele }}(\mathbf{r} ; \mathbf{R}) \Psi^{N}(\mathbf{R})
$$

como $\mathbf{R}$ é mantido fixo, $\epsilon(\mathbf{R})$ e o potencial de repulsão núcleo-núcleo são números, então podemos dividir a equação 2.14 por $\Psi^{\text {ele }}(\mathbf{r} ; \mathbf{R}) \Psi^{N}(\mathbf{R})$,

$$
E(\mathbf{R})=\epsilon(\mathbf{R})+\sum_{A<B}^{M} \frac{Z_{A} Z_{B}}{R_{A B}}
$$

que é o potencial sentido pelos núcleos.

Portanto, resolvendo a equação 2.11 para $\mathbf{R}$, obtemos uma superfície de energia potencial $\mathrm{E}(\mathbf{R})$. Usando a equação 2.13 podemos encontrar a função de onda que descreve o movimento nuclear, $\Psi^{N}(\mathbf{R})$. 
Nas seções seguintes consideraremos apenas o movimento eletrônico dado pela equação (2.11), tornando desnecessário o uso de superescritos para distinguir os termos eletrônicos e nucleares.

\subsection{Método de Hartree-Fock}

A aproximação fundamental da teoria de Hartree-Fock (HF) é que cada elétron se move em um campo elétrico estático, criado por todos os outros elétrons. Fazendo uso dessa aproximação o método de Hartree-Fock busca uma solução aproximada para o estado fundamental de um sistema de elétrons. Essa teoria é a base para todos os outros métodos pós-HF, por isso a sua importância no estudo de sistemas multieletrônicos. O método teve início com a aproximação proposta por Hartree [30, 31 que consiste em considerar a função de onda de um sistema multieletrônico $(\Psi(\mathbf{r}))$ como o produto de funções de onda de um elétron $\left(\varphi_{i}\left(\mathbf{r}_{i}\right)\right)$, equação (2.16). Nessa aproximação, os elétrons de um sistema multieletrônico movem-se de maneira independente.

$$
\Psi(\mathbf{r})=\varphi_{1}\left(\mathbf{r}_{1}\right) \varphi_{2}\left(\mathbf{r}_{2}\right) \ldots \varphi_{N}\left(\mathbf{r}_{N}\right)
$$

As funções de onda de um elétron são chamadas de orbitais, sendo assim os $\varphi_{i}$ são orbitais espaciais e $\mathbf{r}_{i}$ representa as coordenadas espaciais do elétron $i$.

Ainda que a função de onda da equação (2.16) seja autofunção do hamiltoniano de um sistema multi-eletrônico, ela não representa um estado físico, pois não satisfaz ao princípio da exclusão de Pauli [33], que diz que a função de onda que descreve um sistema de muitos elétrons deve ser antissimétrica perante uma troca das coordenadas espaciais e de spin de dois elétrons quaisquer. Para resolver esse problema, em 1930, Fock [32] propôs uma modificação na função de onda multieletrônica proposta por Hartree, sugerindo o uso do determinante de Slater [34] para a construção de uma 
função de onda $\Psi_{0}$,

$$
\Psi_{0}=\frac{1}{\sqrt{N !}}\left|\begin{array}{cccc}
\chi_{1}\left(\mathbf{x}_{1}\right) & \chi_{2}\left(\mathbf{x}_{1}\right) & \ldots & \chi_{N}\left(\mathbf{x}_{1}\right) \\
\chi_{1}\left(\mathbf{x}_{2}\right) & \chi_{2}\left(\mathbf{x}_{2}\right) & \ldots & \chi_{N}\left(\mathbf{x}_{2}\right) \\
\vdots & \vdots & & \vdots \\
\chi_{1}\left(\mathbf{x}_{N}\right) & \chi_{2}\left(\mathbf{x}_{N}\right) & \ldots & \chi_{N}\left(\mathbf{x}_{N}\right)
\end{array}\right|
$$

sendo $\mathrm{N}$ o número total de elétrons e os $\chi^{\prime} s$ funções dependentes das coordenadas espaciais e de spin de um único elétron, chamadas spin-orbitais moleculares [35]. Admitindo que os $\chi^{\prime} s$ são ortonormais, o fator $\frac{1}{\sqrt{N !}}$ é uma constante de normalização para $\Psi_{0}$. Uma notação mais compacta é normalmente encontrada,

$$
\begin{gathered}
\Psi_{0}=\frac{1}{\sqrt{N !}} \operatorname{det}\left(\chi_{1} \chi_{2} \ldots \chi_{N}\right) \\
\Psi_{0}=\left|\chi_{1} \chi_{2} \ldots \chi_{N}\right\rangle
\end{gathered}
$$

Em princípio, as dependências com relação às partes espacial e de spin dos spinorbitais moleculares podem ser separadas,

$$
\chi_{i}\left(\mathbf{x}_{1}\right)=\varphi_{i}\left(\mathbf{r}_{1}\right) \alpha(1)
$$

$\mathrm{Ou}$

$$
\chi_{i}\left(\mathbf{x}_{1}\right)=\varphi_{i}\left(\mathbf{r}_{1}\right) \beta(1)
$$

onde $\alpha$ e $\beta$ representam, respectivamente, spin para cima $(\uparrow)$ e spin para baixo $(\downarrow)$ e os $\varphi^{\prime} s$ são funções somente das coordenadas espaciais de um elétron.

É interessante observar que o uso do determinate de Slater para obter $\Psi_{0}$ garante automaticamente sua antissimetria, o que está de acordo com o princípio de exclusão de Pauli. Com a utilização da função de onda obtida através do determinante de Slater 
(proposta feita por Fock) o método passou a ser chamado método de Hartree-Fock (HF).

O método de HF, além de considerar que a função de onda que descreve um sistema molecular é um determinate de Slater, ele envolve a utilização do princípio variacional [26, 32] para a obtenção dos spin-orbitais moleculares, $\chi^{\prime} s$, que conduzem a um minímo o valor médio do operador hamiltoniano. Desta forma, devemos minimizar o funcional $E(\chi)$, que é o valor esperado do operador hamiltoniano (2.4), calculado no estado $\Psi_{0}$. Pode-se mostrar 35,32 que a dependência deste funcional com os spin-orbitais é dada da seguinte forma:

$$
E(\chi)=\sum_{i}\left\langle\chi_{i}|h| \chi_{i}\right\rangle+\frac{1}{2} \sum_{i, j}\left(\left\langle\chi_{i} \chi_{j} \mid \chi_{i} \chi_{j}\right\rangle-\left\langle\chi_{i} \chi_{j} \mid \chi_{j} \chi_{i}\right\rangle\right)
$$

onde h é o operador de um elétron,

$$
h\left(\mathbf{x}_{i}\right)=-\frac{1}{2} \nabla_{i}^{2}-\sum_{A=1}^{M} \frac{Z_{A}}{r_{i A}}
$$

O segundo membro na equação 2.22 engloba integrais de dois elétrons, em que o primeiro termo é a repulsão coulombiana entre dois elétrons nos orbitais i e j, e o segundo termo é a interação de troca entre os elétrons, nesses orbitais.

$$
\left\langle\chi_{i} \chi_{j} \mid \chi_{k} \chi_{l}\right\rangle=\int \chi_{i}^{*}\left(\mathbf{x}_{1}\right) \chi_{j}^{*}\left(\mathbf{x}_{2}\right) \frac{1}{r_{12}} \chi_{k}\left(\mathbf{x}_{1}\right) \chi_{l}\left(\mathbf{x}_{2}\right) d \mathbf{x}_{1} d \mathbf{x}_{2}
$$

A minimização da equação 2.22 é feita sob a restrição de que os spin-orbitais moleculares permaneçam ortonormais, ou seja,

$$
\left\langle\chi_{i} \mid \chi_{j}\right\rangle-\delta_{i j}=0
$$

Problemas deste tipo, onde condições de vínculo se fazem presentes, podem ser resolvidos utilizando-se a técnica dos multiplicadores indeterminados de Lagrange 36. 
O método consiste em minimizar o funcional

$$
L(\chi)=E(\chi)-\sum_{i, j} \epsilon_{i j}\left(\left\langle\chi_{i} \mid \chi_{j}\right\rangle-\delta_{i j}\right)
$$

onde os coeficientes $\epsilon_{i j}$ são os multiplicadores de Lagrange. Minimizando o funcional, obtemos:

$$
f\left(\mathbf{x}_{1}\right) \chi_{i}\left(\mathbf{x}_{1}\right)=\sum_{j=1}^{N} \epsilon_{i j} \chi_{j}\left(\mathbf{x}_{1}\right)
$$

sendo $f$ o operador de Fock, que é dado por:

$$
f\left(\mathbf{x}_{1}\right)=h\left(\mathbf{x}_{1}\right)+\sum_{j=1}^{N}\left[\mathcal{J}_{j}\left(\mathbf{x}_{1}\right)-\mathcal{K}_{j}\left(\mathbf{x}_{1}\right)\right]
$$

onde h é um operador de uma partícula e no somatório temos os operadores de Coulomb, $\mathcal{J}_{j}$, e de troca, $\mathcal{K}_{j}$. Os operadores $\mathcal{J}_{j}$ e $\mathcal{K}_{j}$ são operadores de duas partículas que produzem integrais do tipo da equação 2.24 e podem ser escritos como:

$$
\begin{aligned}
& \mathcal{J}_{j}\left(\mathbf{x}_{1}\right) \chi_{i}\left(\mathbf{x}_{1}\right)=\left[\int \chi_{j}^{*}\left(\mathbf{x}_{2}\right) \frac{1}{r_{12}} \chi_{j}\left(\mathbf{x}_{2}\right) d \mathbf{x}_{2}\right] \chi_{i}\left(\mathbf{x}_{1}\right) \\
& \mathcal{K}_{j}\left(\mathbf{x}_{1}\right) \chi_{i}\left(\mathbf{x}_{1}\right)=\left[\int \chi_{j}^{*}\left(\mathbf{x}_{2}\right) \frac{1}{r_{12}} \chi_{i}\left(\mathbf{x}_{2}\right) d \mathbf{x}_{2}\right] \chi_{i}\left(\mathbf{x}_{1}\right)
\end{aligned}
$$

Na equação (2.29) o operador de Coulomb descreve a repulsão clássica coulombiana entre duas nuvens eletrônicas. O operador de troca 2.30 faz a mudança entre os elétrons do orbital $j$ e do orbital em que o operador atua e está relacionado com a correlação de troca, ou seja, à correlação no movimento dos elétrons com spins paralelos 32 .

Diagonalizando a matriz $\epsilon$, equação (2.27), através de uma transformação unitária, obteremos um novo conjunto de spin-orbitais, que serão os autovetores do operador de 
Fock, e os $\epsilon_{i}^{\prime} s$ podem ser interpretados como sendo as energias associadas a esses orbitais, onde o operador $f$ representa a energia sentida por um elétron. Após fazermos isso, a equação (2.27) é reescrita da seguinte forma:

$$
f\left(\mathbf{x}_{1}\right) \chi_{i}\left(\mathbf{x}_{1}\right)=\epsilon_{i} \chi_{i}\left(\mathbf{x}_{1}\right)
$$

Conhecida como equação canônica de Hartree-Fock.

Essa é uma equação de autovalores onde $f$, conhecido como operador de Fock, é um operador hermitiano; todos seus autovalores são reais e as autofunções pertencentes a diferentes autovalores são mutuamente ortogonais.

O operador de Fock, $f$, pode ser escrito como:

$$
f\left(\mathbf{x}_{i}\right)=-\frac{1}{2} \nabla_{i}^{2}-\sum_{A=1}^{M} \frac{Z_{A}}{r_{i A}}+v^{H F}\left(\mathbf{x}_{i}\right)
$$

O termo final na equação 2.32 , $v^{H F}$ (definido por $2 \mathcal{J}_{j}-\mathcal{K}_{j}$ ), é o potencial de Hartree-Fock que representa o potencial médio do i-ésimo elétron na presença de todos os outros elétrons. Assim, o método de HF é uma aproximação de campo médio. Por esse motivo, nessa aproximação, perde-se a correlação eletrônica.

A importância do método de HF para a química teórica se dá não somente pelos seus resultados, mas por ser o fundamento para as aproximações posteriores que incluem os efeitos de correlação eletrônica, chamadas de pós-HF.

O procedimento para resolver a equação de HF (2.31) é através de um método iterativo chamado método do campo autoconsistente (Self-Consistent Field - SCF) [38]. O método consiste em escolher spin-orbitais iniciais por tentativa e a partir daí construir o potencial efetico $v^{H F}$ e resolver as equações, possibilitando a obtenção de um novo conjunto de spin-orbitais. A sequencia continua até que se obtenha um conjunto de funções, que comparado ao anterior, não apresente nenhuma mudança. 
Neste ponto considera-se que o cálculo convergiu.

\subsubsection{Equação de Hartree-Fock-Roothaan}

Para átomos, o problema de resolver a equação de Hartree-Fock é simplificado devido à simetria esférica [39]. No caso de moléculas a situação é mais complexa, já que essa simetria é perdida. Portanto, esse procedimento se mostrou computacionalmente inadequado para sistemas moleculares, pela dificuldade de representação dos orbitais moleculares. Esse problema foi resolvido em 1951 quando Roothaan apresentou uma abordagem matricial para a resolução da equação de HF [38]. Nessa formulação matricial do método HF, a essência é expandir os spin-orbitais moleculares em um conjunto de funções base conhecidas. A grande maioria dos cálculos de estrutura eletrônica é baseada na teoria em que aproximamos os orbitais moleculares por uma combinação linear de orbitais atômicos(Linear Combination of Atomic Orbitals, LCAO ) dados a priori.

Eliminando as coordenadas de spin do problema, nós retornamos aos orbitais espaciais, $\phi^{\prime} s$. Considerando moléculas de camada fechada, onde todos os orbitais moleculares são duplamente ocupados, a equação de HF em termos dos orbitais espaciais pode ser escrita como:

$$
f\left(\mathbf{r}_{1}\right) \phi_{i}\left(\mathbf{r}_{1}\right)=\epsilon_{i} \phi_{i}\left(\mathbf{r}_{1}\right)
$$

com o operador de Fock dado por:

$$
f\left(\mathbf{r}_{1}\right)=h\left(\mathbf{r}_{1}\right)+\sum_{j=1}^{N / 2}\left[2 \mathcal{J}_{j}\left(\mathbf{r}_{1}\right)-\mathcal{K}_{j}\left(\mathbf{r}_{1}\right)\right]
$$

onde o fator 2 surge porque, para os mesmos orbitais, coexistem dois elétrons.

Usando um conjunto de orbitais $\mathrm{g}_{\nu}(\mathbf{r})$ podemos expandir os orbitais moleculares, 
$\phi^{\prime} s$, da seguinte forma:

$$
\phi_{i}(\mathbf{r})=\sum_{\nu=1}^{k} C_{\nu i} g_{\nu}(\mathbf{r})
$$

onde $k$ é o número de funções do conjunto e os $\mathrm{C}_{\nu i}$ 's são os coeficientes a serem determinados.

As funções mais utilizadas em cálculos moleculares são as chamadas funções tipo gaussianas, principalmente por causa da facilidade de se calcular integrais utilizando este tipo de função.

Substituindo a expansão 2.35 na equação de Hartree-Fock 2.33), temos:

$$
f\left(\mathbf{r}_{1}\right) \sum_{\nu=1}^{k} C_{\nu i} g_{\nu}\left(\mathbf{r}_{1}\right)=\epsilon_{i} \sum_{\nu=1}^{k} C_{\nu i} g_{\nu}\left(\mathbf{r}_{1}\right)
$$

multiplicando essa equação por $g_{\mu}^{*}\left(\mathbf{r}_{1}\right)$ e integrando, temos:

$$
\sum_{\nu=1}^{k} C_{\nu i}\left\langle g_{\mu}\left(\mathbf{r}_{1}\right)\left|f\left(\mathbf{r}_{1}\right)\right| g_{\nu}\left(\mathbf{r}_{1}\right)\right\rangle=\epsilon_{i} \sum_{\nu=1}^{k} C_{\nu i}\left\langle g_{\mu}\left(\mathbf{r}_{1}\right) \mid g_{\nu}\left(\mathbf{r}_{1}\right)\right\rangle
$$

Definindo os elementos da matriz de superposição,

$$
S_{\mu \nu}=\left\langle g_{\mu}\left(\mathbf{r}_{1}\right) \mid g_{\nu}\left(\mathbf{r}_{1}\right)\right\rangle
$$

e da matriz de Fock,

$$
F_{\mu \nu}=\left\langle g_{\mu}\left(\mathbf{r}_{1}\right)\left|f\left(\mathbf{r}_{1}\right)\right| g_{\nu}\left(\mathbf{r}_{1}\right)\right\rangle
$$

podemos então escrever,

$$
\sum_{\nu=1}^{k} F_{\mu \nu} C_{\nu i}=\epsilon_{i} \sum_{\nu=1}^{k} S_{\mu \nu} C_{\nu i}
$$


Em forma matricial, temos:

$$
\mathbf{F C}=\mathbf{S C} \epsilon
$$

onde $\epsilon$ é uma matriz diagonal que contém as energias orbitais. Esta equação matricial é comumente chamada equação de Hartree-Fock-Roothaan. O que devemos fazer agora é ortogonalizar a matriz $\mathbf{S}$ de modo que a equação de Hartree-Fock-Roothaan tenha a forma de uma equação de autovalores matricial canônica que possa ser resolvida com relativa facilidade. Como S é hermitiana (igual a sua transposta conjugada), ela pode ser diagonalizada por uma transformação unitária. Essa matriz unitária é usada para obter uma nova matriz de Fock e uma nova matriz de coeficientes e obtemos assim a equação de autovalores. Essa nova equação é resolvida ortogonalizando-se a matriz de Fock resultante.

O método de HF trata o problema de interação elétron-elétron de uma forma média, ou como uma interação autoconsistente. Cada elétron participa do potencial que dá origem ao movimento de todos os elétrons. Isto significa que não há um tratamento detalhado onde o movimento de cada elétron é correlacionado com o movimento de todos os outros. A inclusão da contribuição do movimento correlacionado é a principal preocupação dos métodos ab initio. Define-se a contribuição da energia de correlação como a diferença entre a energia exata, não relativística, e a energia de Hartree-Fock [40],

$$
E_{\text {corr }}=E_{\text {exata }}-E_{H F}
$$

É importante mencionar que uma solução regular da equação de Hartree-Fock pode obter até $99 \%$ da energia total exata não relativística. A correlação é obtida ou por uma superposição de configurações ou por tratamentos perturbativos, este último será discutido a seguir. 


\subsubsection{Funções de Base}

Como mostrado na equação 2.35 o orbital molecular pode ser construido através de uma combinação linear de funções de base, onde essas funções são orbitais atômicos. Dois tipos de funções de base são comumentes utilizados para o cálculo de estrutura eletrônica. São elas: funções de Slater (Slater Type Orbitals, STO's) e as funções Gaussianas (Gaussian Type Orbitals, GTO’s). Há também as funções que combinam as funções de Slater e as funções Gaussianas. Esse conjunto de bases, STO-KG consiste em expandir os orbitais do tipo Slater em K funções gaussianas primitivas,

$$
\chi_{m}^{S T O}=\sum_{n}^{K} d_{m n} \chi_{n}^{G T O}
$$

Aqui, a função de base formada pela combinação linear de funções gaussianas é definida como uma base contraída e cada gaussiana que compõem essa combinação linear é definida como primitiva.

Huzinaga [41] e Pople e colaboradores [42], constataram que a melhor combinação entre a precisão e a velocidade para as funções de bases obtidas pela combinação linear de gaussianas foi obtido com $\mathrm{K}=3$. Essa função base, $\mathrm{STO}-3 \mathrm{G}$, ficou conhecida como base mínima ou single - $\zeta$, em que há apenas uma função de base definida para cada tipo de orbital. Embora a escolha da base mínima seja satisfatória para alguns sistemas ela apresenta limitações, nesse sentido, surgem funções de base em que o orbital atômico será representado a partir de duas funções contraídas, esse novo conjunto de funções de base é denominado double $-\zeta$. O mesmo raciocínio é seguido para construir funções multiple - $\zeta$. Há também o conjunto de funções de base split-valence em que os orbitais internos são representados por funções single ou double - $\zeta$ e possuem um número maior de funções de base para os orbitais de valência.

Neste trabalho realizamos cálculos com funções de base que usam o esquema de contração segmentado, utilizado por Pople, em que cada função de base primitiva con- 
tribui somente para uma função contraída. Também realizamos cálculos com as bases de Dunning e colaboradores, em que o esquema de contração utilizado é a contração geral.

A designação utilizado por Pople e colaboradores [43] para representar as funções de base contraídas é:

- 6-31G: É uma função split-valence, em que a região próxima ao núcleo é representada pela contração de seis gaussianas, a região interna dos orbitais da camada de valência é representada pela contração de 3 gaussianas e a parte externa da camada de valência é representada pela contração de uma gaussiana;

- 6-31G*: (*) Indica que foi adicionada uma função tipo-d para polarizar a função tipo- $p$ na base 6-31G;

- 6-31G**: (**) Indica que foi adicionado funções de polarização tipo- $d$ para os átomos pesados e tipo- $p$ para os hidrogênios;

- 6-31+G**: $(+)$ Indica que foi adicionado funções difusas nos átomos pesados;

- 6-31++ $\mathrm{G}^{* *}$ : $(++)$ Indica que foi adicionado funções difusas nos átomos pesados e também nos átomos de hidrogênio.

Na notação para as funções de base de Dunning e colaboradores, temos:

- cc-pVDZ: É uma função onde os expoentes e coeficientes de contração são otimizados variacionalmente, incluindo a correlação eletrônica. Os elétrons da camada de valência são representados por funções double $-\zeta$;

- cc-pVTZ: Possui a mesma descrição anterior sendo que os elétrons da camada de valência são representados por funções triple $-\zeta$ 
- aug-cc-pVTZ: Nessa função de base são acrescentadas funções difusas do tipo $f$, $d, p$ e $s$ para os átomos pesados e funções $d, p$ e $s$ para os átomos de hidrogênio.

Os cálculos em nível HF e pós-HF realizados neste trabalho foram feitos usando o programa Gaussian [44. Na próxima seção discutiremos métodos pós-HF que usam teoria de perturbações para incluir a energia de correlação negligenciada no método HF. Como o nome sugere, pós-HF, esses métodos têm como ponto de partida as aproximações propostas por HF, buscando melhorar os seus resultados.

\subsection{Aproximações Perturbativas}

\subsubsection{Teoria de Perturbação de Rayleigh-Schrödinger}

Dentre as formulações perturbativas básicas, a mais popular é a Teoria de Pertubação de Rayleigh-Schrödinger (TPRS) [35], cuja idéia central é assumir que o hamiltoniano do sistema de interesse pode ser dividido em duas partes:

$$
\mathcal{H}=\mathcal{H}_{0}+\mathcal{V}
$$

onde $\mathcal{H}_{0}$, hamiltoniano não perturbado, difere pouco de $\mathcal{H}$ e os seus autovalores e autovetores são conhecidos,

$$
\mathcal{H}_{(0)}\left|\Psi_{n}^{(0)}\right\rangle=E_{n}^{(0)}\left|\Psi_{n}^{(0)}\right\rangle \quad n=0,1,2,3, \ldots
$$

O problema consiste em resolver a equação de autovalor para o hamiltoniano $\mathcal{H}$,

$$
\mathcal{H}\left|\Psi_{n}\right\rangle=E_{n}\left|\Psi_{n}\right\rangle
$$


onde $n$ indica o estado a ser corrigido. Substituindo a equação (2.44) na (2.46), temos:

$$
\left(\mathcal{H}_{0}+\mathcal{V}\right)\left|\Psi_{n}\right\rangle=E_{n}\left|\Psi_{n}\right\rangle
$$

de maneira que os autovetores e autovalores de $\mathcal{H}$ podem ser obtidos partindo-se dos autovalores e autovetores conhecidos de $\mathcal{H}_{0}$ e dos elementos de matriz de $\mathcal{V}$ na base dos autovetores de $\mathcal{H}_{0}$.

Para que se tenha o ordenamento das correções na energia e na função de onda, é conveniente escrever a equação (2.44) na seguinte forma:

$$
\mathcal{H}=\mathcal{H}_{0}+\lambda \mathcal{V}
$$

onde $\lambda$ é um parâmetro de ordenamento, tal que para $\lambda \rightarrow 0$, o autovalor exato $E_{n}$ tende ao autovalor não perturbado $E_{n}^{(0)}$.

Como o hamiltoniano $\mathcal{H}$ depende de $\lambda$, fica claro que tanto a energia como a função de onda perturbadas dependerão igualmente de $\lambda$.

Expandindo a função exata $\left|\Psi_{n}\right\rangle$ e a energia exata $E_{n}$ em uma série de Taylor em torno de $\lambda=0$, temos:

$$
E_{n}(\lambda)=E_{n}^{(0)}+\lambda\left(\frac{d E_{n}}{d \lambda}\right)_{\lambda=0}+\lambda^{2} \frac{1}{2 !}\left(\frac{d^{2} E_{n}}{d \lambda^{2}}\right)_{\lambda=0}+\ldots
$$

Definindo que:

$$
E_{n}^{(1)}=\left(\frac{d E_{n}}{d \lambda}\right)_{\lambda=0}, \quad E_{n}^{(2)}=\frac{1}{2 !}\left(\frac{d^{2} E_{n}}{d \lambda^{2}}\right)_{\lambda=0}, \ldots
$$

podemos reescrever a equação 2.49,

$$
E_{n}=E_{n}^{(0)}+\lambda E_{n}^{(1)}+\lambda^{2} E_{n}^{(2)}+\ldots
$$


onde $E_{n}^{(j)}$ é a correção de ordem $j$ na energia. Analogamente, podemos escrever a expansão para a função de onda $\Psi_{n}$,

$$
\Psi_{n}=\left|\Psi_{n}^{(0)}\right\rangle+\lambda\left|\Psi_{n}^{(1)}\right\rangle+\lambda^{2}\left|\Psi_{n}^{(2)}\right\rangle+\ldots
$$

onde $\left|\Psi_{n}^{(j)}\right\rangle$ é a correção de ordem $j$ na função de onda.

Aqui vamos supor que o estado não pertubado, $E_{n}^{(0)}$, seja não degenerado e que suas autofunções sejam ortonormais, ou seja,

$$
\left\langle\Psi_{i}^{(0)} \mid \Psi j^{(0)}\right\rangle=\delta_{i j}
$$

Além disso, devemos impor a normalização intermediária,

$$
\left\langle\Psi_{n}^{(0)} \mid \Psi_{n}\right\rangle=1
$$

Agora, as correções na energia e na função de onda são obtidas substituindo-se as equações 2.51) e 2.52 nas equações (2.47) e (2.48), temos:

$$
\begin{array}{r}
\left(\mathcal{H}_{0}+\lambda \mathcal{V}\right)\left(\left|\Psi_{n}^{(0)}\right\rangle+\lambda\left|\Psi_{n}^{(1)}\right\rangle+\lambda^{2}\left|\Psi_{n}^{(2)}\right\rangle+\ldots\right)= \\
\left(E_{n}^{(0)}+\lambda E_{n}^{(1)}+\lambda^{2} E_{n}^{(2)}+\ldots\right)\left(\left|\Psi_{n}^{(0)}\right\rangle+\lambda\left|\Psi_{n}^{(1)}\right\rangle+\lambda^{2}\left|\Psi_{n}^{(2)}\right\rangle+\ldots\right)
\end{array}
$$

Igualando-se os termos de mesma potência em $\lambda$, obtemos: 


$$
\begin{aligned}
\lambda^{0} \rightarrow \mathcal{H}_{0}\left|\Psi_{n}^{(0)}\right\rangle=E_{n}^{(0)}\left|\Psi_{n}^{(0)}\right\rangle & \\
\lambda^{1} \rightarrow\left(\mathcal{H}_{0}-E_{n}^{(0)}\right)\left|\Psi_{n}^{(1)}\right\rangle & =\left(E_{n}^{(1)}-\mathcal{V}\right)\left|\Psi_{n}^{(0)}\right\rangle \\
\lambda^{2} \rightarrow\left(\mathcal{H}_{0}-E_{n}^{(0)}\right)\left|\Psi_{n}^{(2)}\right\rangle & =\left(E_{n}^{(1)}-\mathcal{V}\right)\left|\Psi_{n}^{(1)}\right\rangle+E_{n}^{2}\left|\Psi_{n}^{(0)}\right\rangle \\
\lambda^{3} \rightarrow\left(\mathcal{H}_{0}-E_{n}^{(0)}\right)\left|\Psi_{n}^{(3)}\right\rangle & \left.=\left(E_{n}^{(1)}-\mathcal{V}\right)\left|\Psi_{n}^{(2)}\right\rangle+E_{n}^{2}\left|\Psi_{n}^{(1)}+E_{n}^{3}\right| \Psi_{n}^{(0)}\right\rangle \\
& \vdots \\
\lambda^{n} \rightarrow\left(\mathcal{H}_{0}-E_{n}^{(0)}\right)\left|\Psi_{n}^{(n)}\right\rangle & \left.=\left(E_{n}^{(1)}-\mathcal{V}\right)\left|\Psi_{n}^{(n-1)}\right\rangle+E_{n}^{2}\left|\Psi_{n}^{(n-2)}+\ldots+E_{n}^{n}\right| \Psi_{n}^{(0)}\right\rangle
\end{aligned}
$$

Multiplicando as equações acima por $\left\langle\Psi_{n}^{(0)}\right|$ e utilizando o resultado

$$
\left\langle\Psi_{n}^{(0)}\right|\left(\mathcal{H}_{0}-E_{n}^{(0)}\right)=0
$$

obtemos:

$$
\begin{aligned}
E_{n}^{(1)} & =\left\langle\Psi_{n}^{(0)}|\mathcal{V}| \Psi_{n}^{(0)}\right\rangle \\
E_{n}^{(2)} & =\left\langle\Psi_{n}^{(0)}\left|\mathcal{V}-E_{0}^{(1)}\right| \Psi_{n}^{(1)}\right\rangle \\
E_{n}^{(3)} & =\left\langle\Psi_{n}^{(0)}\left|\mathcal{V}-E_{0}^{(1)}\right| \Psi_{n}^{(2)}\right\rangle-E_{n}^{(2)}\left\langle\Psi_{n}^{(0)} \mid \Psi_{n}^{(1)}\right\rangle \\
& \vdots \\
E_{n}^{(n)} & =\left\langle\Psi_{n}^{(0)}\left|\mathcal{V}-E_{0}^{(1)}\right| \Psi_{n}^{(n-1)}\right\rangle-E_{n}^{(2)}\left\langle\Psi_{n}^{(0)} \mid \Psi_{n}^{(n-2)}\right\rangle-\ldots-E_{n}^{(n-1)}\left\langle\Psi_{n}^{(0)} \mid \Psi_{n}^{(1)}\right\rangle
\end{aligned}
$$

Assim, os conjuntos de equações 2.56 e 2.58 permitem, em princípio, obter as correções em qualquer ordem na função de onda e na energia. As expressões contidas na equação 2.58 podem ser simplificadas utilizando-se a normalização intermediária 
$(2.54)$

$$
\begin{aligned}
E_{n}^{(1)} & =\left\langle\Psi_{n}^{(0)}|\mathcal{V}| \Psi_{n}^{(0)}\right\rangle \\
E_{n}^{(2)} & =\left\langle\Psi_{n}^{(0)}|\mathcal{V}| \Psi_{n}^{(1)}\right\rangle \\
E_{n}^{(3)} & =\left\langle\Psi_{n}^{(0)}|\mathcal{V}| \Psi_{n}^{(2)}\right\rangle \\
& \vdots \\
E_{n}^{(n)} & =\left\langle\Psi_{n}^{(0)}|\mathcal{V}| \Psi_{n}^{(n-1)}\right\rangle
\end{aligned}
$$

As equações 2.59 nos mostram que para se obter a energia corrigida até a ordem $j$ é necessário o conhecimento da função de onda até ordem $j-1$. Na verdade, é possível mostrar que o conhecimento da correção de ordem $j$, na função de onda, nos permite determinar a correção na energia até a ordem $2 j+1$ [40].

Como nem sempre é possível obter a solução exata $\left|\Psi_{n}^{(j)}\right\rangle$ para o conjunto de equações diferenciais não homogêneas dadas na equação (2.57), o que se faz é uma expansão das funções de onda perturbadas $\left|\Psi_{n}^{(j)}\right\rangle$, para isso usa-se como base nessa expansão o conjunto completo gerado pelas autofunções do hamiltoniano não perturbado, $\left|\Psi_{i}^{(0)}\right\rangle$,

$$
\left|\Psi_{n}^{(j)}\right\rangle=\sum_{i} c_{i}^{j}\left|\Psi_{i}^{(0)}\right\rangle
$$

Para ilustrar as correções na função de onda e na energia, vamos obter a correção de primeira ordem, $j=1$, para a função de onda e a correção de segunda ordem para a energia. Multiplicando por $\left|\Psi_{k}^{(0)}\right\rangle$ a expressão para potência $1, \lambda^{1}$, na equação 2.56), obtemos:

$$
\left\langle\Psi_{k}^{(0)}\left|\left(\mathcal{H}_{0}-E_{n}^{(0)}\right)\right| \Psi_{n}^{(1)}\right\rangle=\left\langle\Psi_{k}^{(0)}\left|\left(E_{n}^{(1)}-\mathcal{V}\right)\right| \Psi_{n}^{(0)}\right\rangle
$$

usando o fato de que $\mathcal{H}_{0}$ é hermitiano e substituindo a equação 2.60 na 2.61), fazendo 
$j=1$, temos:

$$
\left(E_{k}^{(0)}-E_{n}^{(0)}\right)\left\langle\Psi_{k}^{(0)}\left|\sum_{i} c_{i}^{(1)}\right| \Psi_{i}^{(0)}\right\rangle=E_{n}^{(1)}\left\langle\Psi_{k}^{(0)} \mid \Psi_{n}^{(0)}\right\rangle-\left\langle\Psi_{k}^{(0)}|\mathcal{V}| \Psi_{n}^{(0)}\right\rangle
$$

Usando o fato de que os autovetores da hamiltoniana não perturbada são ortonormais, equação (2.53), obtemos:

$$
\left(E_{k}^{(0)}-E_{n}^{(0)}\right) \sum_{i} c_{i}^{(1)} \delta_{k i}=-\left\langle\Psi_{k}^{(0)}|\mathcal{V}| \Psi_{n}^{(0)}\right\rangle
$$

$\log 0$

$$
c_{k}^{(1)}=\frac{\left\langle\Psi_{k}^{(0)}|\mathcal{V}| \Psi_{n}^{(0)}\right\rangle}{E_{n}^{(0)}-E_{k}^{(0)}}
$$

substituindo a equação (2.64) na equação (2.60), fazendo $j=1$, obtemos a correção em primeira ordem para a função de onda:

$$
\left|\Psi_{n}^{(1)}\right\rangle=\sum_{k} \frac{\left\langle\Psi_{k}^{(0)}|\mathcal{V}| \Psi_{n}^{(0)}\right\rangle}{E_{n}^{(0)}-E_{k}^{(0)}}\left|\Psi_{k}^{(0)}\right\rangle
$$

A correção em segunda ordem na energia é obtida substituindo a equação 2.65 na expressão para $E_{n}^{(2)}$ na equação 2.59,

$$
E_{n}^{(2)}=\sum_{k \neq n} \frac{\left|\left\langle\Psi_{k}^{(0)}|\mathcal{V}| \Psi_{n}^{(0)}\right\rangle\right|^{2}}{E_{n}^{(0)}-E_{k}^{(0)}}
$$

\subsubsection{Teoria de Perturbação Møller-Plesset}

A forma usual de perturbação em química quântica usa TPRS com a partição do hamiltoniano eletrônico proposta por Møller-Plesset [45], e é comumente chamada teoria de perturbação Møller-Plesset (MP) 46]. 
A teoria MP considera como o hamiltoniano não perturbado, $\mathcal{H}_{0}$, como a soma de operadores de Fock tal como definido na equação 2.28,

$$
\mathcal{H}_{0}=\sum_{i=1}^{N} f(i)
$$

A perturbação, $\mathcal{V}$, é então obtida pela diferença entre o hamiltoniano molecular dado pela equação (2.4), $\mathcal{H}$, e o hamiltoniano não perturbado, $\mathcal{H}_{0}$,

$$
\mathcal{V}=\mathcal{H}-\mathcal{H}_{0}=\sum_{i<j} \frac{1}{r_{i j}}-\sum_{i} v^{H F}(i)
$$

onde

$$
v^{H F}(i)=\sum_{j}\langle j \| j\rangle=\sum_{j}\left[\mathcal{J}_{j}(i)-\mathcal{K}_{j}(i)\right]
$$

Com $\mathcal{H}_{0}$ definido pela equação (2.67), temos que a função de onda Hartree-Fock 2.19, $\Psi_{0}$, é autovetor de $\mathcal{H}_{0}$ com autovalor $E_{0}^{(0)}$, dado pela soma das energias orbitais de todos os spin-orbitais,

$$
\begin{aligned}
\mathcal{H}_{0} \Psi_{0} & =\sum_{i=1}^{N} f(i)\left|\chi_{1} \chi_{2} \ldots \chi_{N}\right\rangle \\
& =\left(\epsilon_{1}+\epsilon_{2}+\ldots+\epsilon_{N}\right)\left|\chi_{1} \chi_{2} \ldots \chi_{N}\right\rangle \\
& =E_{0}^{(0)} \Psi_{0}
\end{aligned}
$$

com

$$
E_{0}^{(0)}=\sum_{c=1}^{N} \epsilon_{c}
$$

Usando as expressões gerais para TPRS podemos calcular as várias correções para um sistema de $\mathrm{N}$ elétrons. A correção em primeira ordem para a energia do 
estado fundamental, $E_{0}^{(1)}$, é dada por:

$$
E_{0}^{(1)}=\left\langle\Psi_{0}|\mathcal{V}| \Psi_{0}\right\rangle=\left\langle\Psi_{0}\left|\sum_{i<j} \frac{1}{r_{i j}}\right| \Psi_{0}\right\rangle-\left\langle\Psi_{0}\left|\sum_{i, j=1}\left[\mathcal{J}_{j}(i)-\mathcal{K}_{j}(i)\right]\right| \Psi_{0}\right\rangle
$$

Usando as regras de Condo-Slater 46, 47, temos:

$$
\begin{gathered}
\left\langle\Psi_{0}\left|\sum_{i<j} \frac{1}{r_{i j}}\right| \Psi_{0}\right\rangle=\frac{1}{2} \sum_{i, j=1}^{N}\left\langle\chi_{i} \chi_{j} \| \chi_{i} \chi_{j}\right\rangle \\
\left\langle\Psi_{0}\left|\sum_{i, j=1}\left[\mathcal{J}_{j}(i)-\mathcal{K}_{j}(i)\right]\right| \Psi_{0}\right\rangle=\sum_{i, j=1}^{N}\left\langle\chi_{i} \chi_{j} \| \chi_{i} \chi_{j}\right\rangle
\end{gathered}
$$

com

$$
\left\langle\chi_{i} \chi_{j} \| \chi_{i} \chi_{j}\right\rangle=\left\langle\chi_{i} \chi_{j} \mid \chi_{i} \chi_{j}\right\rangle-\left\langle\chi_{i} \chi_{j} \mid \chi_{j} \chi_{i}\right\rangle
$$

e a correção de primeira ordem é:

$$
E_{0}^{(1)}=-\frac{1}{2} \sum_{i, j=1}^{N}\left\langle\chi_{i} \chi_{j} \| \chi_{i} \chi_{j}\right\rangle
$$

Logo, a energia para o estado fundamental de um sistema com N elétrons corrigida até primeira ordem é dada por:

$$
E_{0}=E_{0}^{(0)}+E_{0}^{(1)}=\sum_{i=1}^{N} \epsilon_{i}-\frac{1}{2} \sum_{i, j=1}^{N}\left\langle\chi_{i} \chi_{j} \| \chi_{i} \chi_{j}\right\rangle=E_{H F}
$$

que é a energia Hartree-Fock para o estado fundamental. Portanto, a energia de Hartree-Fock é correta até primeira ordem na MP. A correlação só se apresenta a partir da segunda ordem:

$$
E_{\text {corr }}=E_{0}^{(2)}+E_{0}^{(3)}+\ldots
$$


A equação (2.66), que dá a correção em segunda ordem na energia, no caso da partição de Møller-Plesset, exige o cálculo de elementos de matriz envolvendo o estado de referência de Hartree-Fock $\left|\Psi_{0}\right\rangle$ e as excitações $\left\{\left|\Psi_{a}^{r}\right\rangle,\left|\Psi_{a b}^{r s}\right\rangle, \ldots,\left|\Psi_{a b \ldots . . .}^{r s \ldots}\right\rangle\right.$. Aqui, $\left|\Psi_{a}^{r}\right\rangle$ significa que são realizadas apenas excitações simples (a excitação eletrônica ocorre do orbital ocupado $a$ para o orbital virtual $r$ ), já o autovetor $\left|\Psi_{a b}^{r s}\right\rangle$ leva em conta excitações duplas e assim sucessivamente. Considerando apenas excitações simples, temos:

$$
\left\langle\Psi_{0}|\mathcal{V}| \Psi_{a}^{r}\right\rangle=\left\langle\Psi_{0}|\mathcal{H}| \Psi_{a}^{r}\right\rangle-\left\langle\Psi_{0}\left|\mathcal{H}_{0}\right| \Psi_{a}^{r}\right\rangle
$$

onde a primeira integral é nula pelo teorema de Brillouin [26] e a segunda parcela dá,

$$
\left\langle\Psi_{0}\left|\mathcal{H}_{0}\right| \Psi_{a}^{r}\right\rangle=\sum_{j} \epsilon_{j}\left\langle\Psi_{0} \mid \Psi_{a}^{r}\right\rangle=0, \quad\left\langle\Psi_{0} \mid \Psi_{a}^{r}\right\rangle=0
$$

o que resulta em

$$
\left\langle\Psi_{0}|\mathcal{V}| \Psi_{a}^{r}\right\rangle=0
$$

Assim, as excitações simples não contribuem para a correção de segunda ordem.

Para excitações triplas e superiores obtém-se que:

$$
\left\langle\Psi_{0}|\mathcal{V}| \Psi_{a b c}^{r s t}\right\rangle=\left\langle\Psi_{0}|\mathcal{V}| \Psi_{a b \ldots}^{r s \ldots}\right\rangle=0
$$

pois $\mathcal{V}$ contém apenas operadores de uma partícula $\left(V^{H F}(i)\right)$ e duas partículas $\left(1 / r_{i j}\right)$. Logo, essas excitações não contribuirão para a correção de segunda ordem.

Pelo que foi analisado anteriormente, observa-se que a correção de segunda ordem da energia do estado fundamental usando MP requer somente a inclusão de excitações duplas,

$$
E_{0}^{(2)}=\sum_{a<b} \sum_{r<s} \frac{\left|\left\langle\Psi_{0}|\mathcal{V}| \Psi_{a b}^{r s}\right\rangle\right|^{2}}{E_{0}^{(0)}-E_{a b}^{r s}}
$$


onde o valor do numerador é obtido usando a regra de Condo-Slater

$$
\left\langle\Psi_{0}|\mathcal{V}| \Psi_{a b}^{r s}\right\rangle=\langle a b \| r s\rangle
$$

Logo,

$$
E_{0}^{(2)}=\sum_{a<b} \sum_{r<s} \frac{|\langle a b \| r s\rangle|^{2}}{\epsilon_{a}+\epsilon_{b}-\epsilon_{r}-\epsilon_{s}}
$$

A equação (2.85) pode ser escrita como uma soma livre notando que a soma é simétrica em $a$ e $b$ e em $r$ e $s$, e que se anula se $a=b$ ou $r=s$. Assim,

$$
E_{0}^{(2)}=\frac{1}{4} \sum_{a<b} \sum_{r<s} \frac{|\langle a b \| r s\rangle|^{2}}{\epsilon_{a}+\epsilon_{b}-\epsilon_{r}-\epsilon_{s}}
$$

conhecida como forma de Brandow [48].

Neste trabalho realizamos cálculos usando a teoria de perturbação MP até segunda ordem, que é denotada por MP2. Vale lembrar que por não ser fundamentada no princípio variacional, as energias obtidas utilizando MP podem ser inferiores à energia exata.

\subsection{Teoria do Funcional da Densidade}

Uma outra forma de resolver a equação de Schrödinger para um sistema multieletrônico é usando a sua densidade eletrônica. A aplicação da Teoria do Funcional da Densidade (DFT) em átomos, sólidos e moléculas tem crescido bastante e, nas últimas décadas, tem se mostrado um importante método para o cálculo de propriedades eletrônicas e estruturais para o estado fundamental. A grande vantagem dessa metodologia, quando comparada com os métodos ab inito padrões, que são baseados no método Hartree-Fock, é apresentar um custo computacional relativamente baixo. 
O uso da densidade eletrônica $\rho(\mathbf{r})$ como variável básica foi legitimada com a publicação dos teoremas de Hohemberg e Kohn (HK) em 1964 sobre gás de elétrons não homogêneos [49], tornando explícita a repulsão elétron-elétron de Coulomb e definindo uma nova função universal $F[\rho]$.

Na DFT, os elétrons interagem entre si e com um potencial externo, $v(\mathbf{r})$. O primeiro teorema de HK estabelece que esse potencial externo é um funcional único, a menos de uma constante, da densidade eletrônica $\rho(\mathbf{r})$. Isso implica que a densidade eletrônica de um sistema determina o potencial externo e o número de elétrons, $\mathrm{N}$, e consequentemente, o hamiltoniano do sistema. Onde esse hamiltoniano para um sistema multieletrônico é escrito como:

$$
\mathcal{H}=\mathcal{T}+\mathcal{U}+\mathcal{V}
$$

onde $\mathcal{T}$ é o operador energia cinética; $\mathcal{U}$, é o operador de repulsão elétron-elétron, que inclui a repulsão Coulombiana e todos os termos não clássicos (troca e correlação); e $\mathcal{V}=\sum_{i}^{N} v\left(r_{i}\right)$, é o potencial externo sentido pelos elétrons, que para moléculas é devido as cargas dos núcleos.

A energia total do sistema é então obtida pelo valor esperado do hamiltoniano, $\langle\Psi|\mathcal{H}| \Psi\rangle$. E essa energia é um funcional da densidade eletrônica $\rho(\mathbf{r})$,

$$
\begin{aligned}
E[\rho] & =\langle\Psi|\mathcal{T}+\mathcal{U}+\mathcal{V}| \Psi\rangle \\
& =\langle\Psi|\mathcal{T}+\mathcal{U}| \Psi\rangle+\langle\Psi|\mathcal{V}| \Psi\rangle \\
& =F[\rho]+\int v(\mathbf{r}) \rho(\mathbf{r}) d^{3} r
\end{aligned}
$$

onde $F[\rho]$ é um funcional universal, ou seja, sua dependência com a densidade é a mesma para todos sistemas que possuam a mesma interação elétron-elétron.

O segundo teorema de HK obedece ao princípio variacional e estabelece que, havendo qualquer aproximação da densidade eletrônica, $\tilde{\rho}(\mathbf{r})$, tal que $\tilde{\rho}(\mathbf{r}) \geq 0$ e 
$\int \tilde{\rho}(\mathbf{r}) d^{3} r=N$, a energia total será sempre maior ou igual a energia exata do sistema, ou seja,

$$
E[\tilde{\rho}]=F[\tilde{\rho}]+\int v(\mathbf{r}) \tilde{\rho}(\mathbf{r}) d^{3} r \geq E[\rho]=E_{0}
$$

onde $\tilde{\rho}(\mathbf{r})$ define seu próprio $\tilde{v}(\mathbf{r})$ e, consequentemente, o hamiltoniano $\tilde{H}$ e $\tilde{\Psi}\left(r_{1}, r_{2}, \ldots, r_{N}\right)$. Em termos, o segundo teorema diz que a energia do estado fundamental do sistema pode ser obtida minimizando a energia total com relação a densidade.

Os teoremas de HK nos garantem que a energia do estado fundamental, para um sistema multieletrônico, pode ser obtida através da sua densidade mas não nos ensina como isso deve ser feito na prática. Um procedimento para resolver essa questão foi proposto por Kohn e Sham em 1965 [50]. A idéia nesse procedimento é construir um sistema auxiliar de partículas independentes, como referência, que possua a mesma densidade do sistema interagente. Na seção seguinte fazemos uma discussão sobre a aproximação de Kohn-Sham (KS).

\subsubsection{Equações de Kohn-Sham}

A determinação da energia do estado fundamental de um sistema multieletrônico, sob um potencial externo, é feita usando a aproximação de Konh-Sham, que utiliza um sistema de partículas independentes como referência.

Kohn e Sham reescreveram a equação 2.90 tornando explícita a repulsão elétronelétron de Coulomb e definindo um novo funcional universal $G[\rho]$ :

$$
E[\rho]=G[\rho]+\frac{1}{2} \iint \frac{\rho(\mathbf{r}) \rho\left(\mathbf{r}^{\prime}\right)}{\left|\mathbf{r}-\mathbf{r}^{\prime}\right|} d^{3} r d^{3} r^{\prime}+\int \rho(\mathbf{r}) v(\mathbf{r}) d^{3} r
$$

onde

$$
G[\rho]=T_{s}[\rho]+E_{x c}[\rho]
$$


sendo $T_{s}[\rho]$ o funcional energia cinética de um sistema de elétrons que não interagem tendo a mesma densidade eletrônica do sistema de elétrons que interagem. Dessa forma, $E_{x c}[\rho]$ inclui não só o termo de interação elétron-elétron não-clássico (troca e correlação) mas também a parte residual da energia cinética, $T[\rho]-T_{s}[\rho]$, em que $T[\rho]$ é o funcional energia cinética exata para o sistema de elétrons que interagem. É interessante ressaltar que a fórmula funcional exata para $E_{x c}[\rho]$ não é simples, e nem mesmo conhecida. É nesse funcional que se concentram as aproximações da DFT prática.

Assim como no método Hartree-Fock, a idéia aqui é minimizar o funcional $E[\rho]$ sujeito ao vínculo de que a carga eletrônica total seja fixa, ou seja,

$$
\int \rho(\mathbf{r}) d^{3} r=N
$$

Fazendo a minimização, com a condição de extremo, incluindo o vínculo, cairemos em uma equação de autovalores dada por (ver referência [26]),

$$
\begin{aligned}
\left(-\frac{1}{2} \nabla^{2}+v^{K S}[\rho]\right) \Psi_{i}(\mathbf{r}) & =\epsilon_{i} \Psi_{i}(\mathbf{r}) \\
\text { оu } & \\
h^{K S} \Psi_{i}(\mathbf{r}) & =\epsilon_{i} \Psi_{i}(\mathbf{r})
\end{aligned}
$$

A equação (2.96) é conhecida como equação de Kohn-Sham (KS) e $h^{K S}$ é o hamiltoniano de KS (operador de uma partícula). O potencial efetivo de KS para uma partícula, $v^{K S}$, é dado por:

$$
v^{K S}=v(\mathbf{r})+\int \frac{\rho\left(\mathbf{r}^{\prime}\right)}{\left|\mathbf{r}-\mathbf{r}^{\prime}\right|} d^{3} r^{\prime}+v_{x c}(\rho)
$$

O primeiro termo na equação (2.97), $v(\mathbf{r})$, é o potencial externo (que para moléculas é devido às cargas dos núcleos). Em seguida vem o potencial de Hartree, que 
descreve a interação eletrostática entre os elétrons. Por último nós temos o potencial de troca-correlação $v_{x c}$, que inclui todos os efeitos não triviais de muitos corpos. Esse termo pode ser escrito como uma derivada funcional do funcional energia de trocacorrelação, $E_{x c}$ (ver referência $[26 \mid$ ),

$$
v_{x c}[\rho]=\frac{\delta E_{x c}}{\delta \rho}
$$

Para obtermos $v^{K S}$, pela equação (2.97), é necessário conhecermos $v_{x c}$. Portanto, da equação (2.98) observa-se que é preciso fazermos uma escolha a priori do funcional troca-correlação, $E_{x c}[\rho]$. Se o funcional energia de troca-correlação, $E_{x c}[\rho]$, fosse conhecido exatamente, a resolução da equação de Kohn-Sham nos forneceria a densidade exata para um sistema de muitos corpos interagentes. Porém, $E_{x c}[\rho]$ é uma quantidade bastante complicada e deve ser aproximada para qualquer aplicação prática do esquema Konh-Shan.

A primeira aproximação para o termo $E_{x c}[\rho]$ foi proposta por Kohn e Shan em 1965 [50]. Essa aproximação é chamada de Aproximação Local da Densidade, LDA (do inglês, Local Density Approximation),

$$
E_{x c}^{L D A}[\rho]=\int \rho(\mathbf{r}) \epsilon_{x c}^{h}(\rho(\mathbf{r})) d^{3} r
$$

onde $\epsilon_{x c}^{h}(\rho)$ é a energia de troca-correlação, por elétron, de um gás de elétrons homogêneo de densidade $\rho=\rho(\mathbf{r})$. Nessa aproximação o funcional $E_{x c}[\rho]$ é local, ou seja, a densidade de energia num ponto $\mathbf{r}$ só dependerá da densidade eletrônica existente no mesmo ponto . Apesar de ser, por construção, uma aproximação local, a LDA produz bons resultados mesmo para sistemas não homogêneos, como átomos ou moléculas pequenas.

Como em sistemas reais a densidade não é homogênea, há um refinamento no método LDA, que é expressar o funcional $E_{x c}[\rho]$ em termos do gradiente da densidade 
de carga total. Essa aproximação é conhecida como expansão generalizada em termos de gradientes GGA (do inglês, Generalized Gradient Approximation), que tem a seguinte fórmula geral [51]:

$$
E_{x c}^{G G A}[\rho]=\int f(\rho(\mathbf{r}), \nabla \rho(\mathbf{r})) d^{3} r
$$

Existem várias propostas para o funcional $E_{x c}^{G G A}$, as mais utilizadas, atualmente, são baseadas nos trabalhos de Perdew-Burke-Erzenhof [52], de Lee-Yang-Parr [53,54], de Perdew e Wang [55], de Perdew [56] e de Becke [57].

Vários desses funcionais energia de troca-correlação foram desenvolvidos de forma a atender parte das restrições que o funcional exato deve possuir como, por exemplo, o comportamento assintótico para grandes distâncias. Esses funcionais apresentam, de modo geral, alguns parâmetros que são ajustados de maneira a reproduzir a energia de troca-correlação exata para alguns sistemas bem estudados, como um gás de elétrons uniforme, ou são ajustados para reproduzir a energia de troca-correlação obtida por outros métodos quânticos. No entanto, há funcionais que são puros como, por exemplo, PW91 (Perdew-Wang) [58] e PBE (Perdew-Burke-Erzenhof) 52].

Há também o funcional híbrido Hartree-Fock/DFT que foi proposto para incorporar a não localidade do método Hartree-Fock na DFT. A idéia dos métodos híbridos é a de incluir parte do termo de troca exato do método Hartree-Fock no funcional GGA.

Na linha dos funcionais híbridos, nós realizamos cálculos usando dois funcionais híbridos distintos: B3LYP (Becke's three-parameter exchange + Lee-Yang-Parr correlation) 54,59 e BHandHLYP (Becke's half-and-half exchange + Lee-Yang-Parr correlation) 600. A energia de troca-correlação, $E_{x c}$, para os dois funcionais difere apenas no termo de troca (exchange). O funcional B3LYP é composto por uma mistura contendo $20 \%$ de Hartree-Fock para a densidade de troca enquanto que o funcional 
BHandHLYP incorpora 50\% de Hartree-Fock para a densidade de troca.

A energia de troca-correlação para o funcional B3LYP é escrita como:

$$
E_{x c}=E_{x c}^{L D A}+a_{0}\left(E_{x}^{H F}-E_{x}^{L D A}\right)+a_{x}\left(E_{x}^{G G A}-E_{x}^{L D A}\right)+a_{c}\left(E_{c}^{G G A}-E_{c}^{L D A}\right)
$$

onde $a_{0}=0.20, a_{x}=0.72$, e $a_{c}=0.81$ são três parâmetros empíricos determinados pelo ajuste dos valores previstos para um conjunto de energias de atomização, potenciais de ionização, próton afinidade e energias atômicas. Os termos, $E_{x}^{G G A}$ e $E_{c}^{G G A}$ correspondem ao funcional de troca Becke88 [57] e ao funcional de correlação de LeeYang-Parr [53], respectivamente. O termo $E_{c}^{L D A}$ corresponde a aproximação proposta por Vosko-Wilk-Nusair (VWN) [61] para o funcional de correlação.

O funcional BHandHLYP é dado por:

$$
E_{x c}=0.5^{*} E_{x}^{H F}+0.5^{*} E_{x}^{B}+1.0^{*} E_{c}^{L Y P}
$$

onde $E_{x}^{H F}$ corresponde ao termo de troca Hartree-Fock, $E_{x}^{B}$ corresponde ao funcional de troca Becke88, e $E_{c}^{L Y P}$ corresponde ao funcional de correlação proposto por LeeYang-Parr.

\subsubsection{DFT Dependente do Tempo (TD-DFT)}

A DFT tem se mostrado bastante eficiente na descrição do estado fundamental de sistemas atômicos e moleculares. Devido ao sucesso da aplicação da DFT em estados estacionários, tem surgido o interesse em descrever estados dependentes do tempo (TD- Time Dependent) em termos do funcional da densidade. Problemas como processos de espalhamento atômico [62 e nuclear [63] e fotoabsorção em átomos 64 têm sido tratados com sucesso nessa abordagem. 
No trabalho de 1984, Runge e Gross [65] generalizaram o teorema de HohenbergKohn para o caso da equação de Schrödinger dependente do tempo,

$$
i \frac{\partial \Phi(\{\mathbf{r}\}, t)}{\partial t}=\mathcal{H}(\{\mathbf{r}\}, t) \Phi(\{\mathbf{r}\}, t), \quad \Phi\left(\{\mathbf{r}\} t_{0}\right)=\Phi_{0}
$$

onde $\{\mathbf{r}\}=\left\{\mathbf{r}_{1}, \ldots, \mathbf{r}_{N}\right\}$ é o conjunto de coordenada espacial de um sistema de $N$ elétrons. O hamiltoniano, $\mathcal{H}(\{\mathbf{r}\}, t)=\mathcal{T}(\{\mathbf{r}\})+\mathcal{W}(\{\mathbf{r}\})+\mathcal{V}(\{\mathbf{r}\}, t)$, consiste na energia cinética,

$$
\mathcal{T}(\{\mathbf{r}\})=-\frac{1}{2} \sum_{i=1}^{N} \nabla_{i}^{2}
$$

na interação elétron-elétron,

$$
\mathcal{W}(\{\mathbf{r}\})=\frac{1}{2} \sum_{i \neq j}^{N} \frac{1}{\left|\mathbf{r}_{i}-\mathbf{r}_{j}\right|}
$$

e num potencial externo,

$$
\mathcal{V}(\{\mathbf{r}\}, t)=\sum_{i=1}^{N} v_{\text {ext }}\left(\mathbf{r}_{i}, t\right)
$$

Runge-Gross demonstraram que existe uma correspondência unívoca entre o potencial externo dependente do tempo $v(\mathbf{r}, t)$ e a densidade eletrônica $\rho(\mathbf{r}, t)$ para sistemas de muitos corpos que evoluam de um estado inicial fixo, este é o teorema central da TD-DFT, também conhecido como teorema de Runge-Gross [66]. Este resultado implica que, se a única informação existente sobre o sistema for a sua densidade eletrônica, nós podemos determinar o potencial externo que produz essa densidade. Definido o potencial externo pode-se então resolver a equação de Schrödinger dependente do tempo, e assim obter todas as propriedades do sistema.

De posse do teorema de Runge-Gross é possível construir um esquema de KohnSham dependente do tempo. Este esquema obedecerá a equação de Schrödinger de- 
pendente do tempo,

$$
i \frac{\partial}{\partial t} \phi_{i}(\mathbf{r}, t)=h_{K S}(\mathbf{r}, t) \phi_{i}(\mathbf{r}, t)
$$

onde $h_{K S}$ é o hamiltoniano de Kohn-Sham e é definido como:

$$
h_{K S}(\mathbf{r}, t)=-\frac{\nabla^{2}}{2}+v_{K S}[\rho](\mathbf{r}, t)
$$

Por construção, a densidade do sistema interagente pode ser determinada a partir dos orbitais de Kohn-Sham, $\phi_{i}$,

$$
\rho(\mathbf{r}, t)=\sum_{i=1}^{N}\left|\phi_{i}(\mathbf{r}, t)\right|^{2}
$$

Como no esquema Kohn-Sham para o estado excitado, o potencial de Kohn-Sham dependente do tempo é normalmente escrito como a soma de três termos,

$$
v_{K S}(\mathbf{r}, t)=v_{\text {ext }}(\mathbf{r}, t)+v_{\text {Hartree }}[\rho](\mathbf{r}, t)+v_{x c}[\rho](\mathbf{r}, t)
$$

o primeiro termo é o potencial externo, o segundo representa a interação eletrostática entre elétrons,

$$
v_{\text {Hartree }}(\mathbf{r}, t)=\int d^{3} r^{\prime} \frac{\rho(\mathbf{r}, t)}{\left|\mathbf{r}-\mathbf{r}^{\prime}\right|}
$$

O terceiro termo na equação 2.110, o potencial de troca-correlação $x c$, inclui todos os efeitos não triviais de muito corpos, e tem uma dependência funcional, extremamente complexa, com a densidade. Esta dependência é não local, tanto no espaço quanto no tempo, ou seja, o potencial num tempo $t$ e uma posição $\mathbf{r}$ dependerá da densidade em todas as outras posições e em todos os instantes de tempo. O que se faz então é separar do potencial $v_{K S}$ em dois termos bem conhecidos, o potencial externo e o potencial de Hartree, e o potencial $x c$ é geralmente aproximado usando argumentos físicos e matemáticos. Portanto, a qualidade dos resultados obtidos dependerá da 
qualidade da aproximação usada. Assim como na teoria do funcional da densidade para o estado fundamental, esta é a aproximação fundamental na TD-DFT.

Das várias maneiras de se implementar a DFT dependente do tempo, a que tem tido maior sucesso faz uso da Teoria de Resposta Linear.

A chave nesse processo é a função de resposta linear da densidade $\chi\left(\mathbf{r}, \mathbf{r}^{\prime}, \omega\right)$ que conecta a perturbação externa $\delta v(\mathbf{r}, \omega)$ à perturbação resultante na densidade eletrônica $\delta \rho(\mathbf{r}, \omega)$ através de

$$
\delta \rho(\mathbf{r}, \omega)=\int \chi\left(\mathbf{r}, \mathbf{r}^{\prime}, \omega\right) \chi\left(\mathbf{r}, \mathbf{r}^{\prime}, \omega\right) d \mathbf{r}^{\prime}
$$

A função resposta da densidade pode ser escrita como 66

$$
\chi\left(\mathbf{r}, \mathbf{r}^{\prime}, \omega\right)=\lim _{\eta \rightarrow 0} \sum_{k}\left[\frac{\langle 0|\rho(\mathbf{r})| k\rangle\left\langle k\left|\rho\left(\mathbf{r}^{\prime}\right)\right| 0\right\rangle}{\omega-\left(E_{k}-E_{0}\right)+i \eta}-\frac{\left\langle 0\left|\rho\left(\mathbf{r}^{\prime}\right)\right| k\right\rangle\langle k|\rho(\mathbf{r})| 0\rangle}{\omega+\left(E_{k}-E_{0}\right)+i \eta}\right]
$$

sendo $\rho$ o operador densidade e os estados $|k\rangle$ de energia $E_{K}$ formam um conjunto completo, $\eta$ é um número infinitésimal positivo. Os polos da função resposta da densidade correspondem às energias de excitação do sistema e os numeradores estão relacionados às correspondentes forças de oscilador. A partir disso chega-se a uma equação de autovalores (não linear) que determina as energias de excitação através dos orbitais de Kohn-Sham.

\subsection{Ajuste das Cargas Parciais - Método Chelpg}

As cargas parciais são de fundamental importância na avaliação de interações intermoleculares em métodos de mecânica molecular, dinâmica molecular e simulações de Monte Carlo, principalmente de moléculas polares. Nesse sentido, o conceito químico de populações eletrônicas atômicas, ou cargas atômicas parciais é muito útil e de grande 
significância. O conceito de cargas pontuais centradas em átomos implica na aproximação do modelo de cargas pontuais, onde as cargas representam termos monopolares centrados nos átomos em uma expansão multipolar da densidade de carga. Consequentemente, as cargas atômicas fornecem uma representação mais simples possível da densidade de carga de uma molécula.

Ao contrário da densidade eletrônica, as cargas atômicas não podem ser calculadas teoricamente de uma maneira única, pois essas não são valores esperados da função de onda, isto é, não são observáveis. Deste modo, todos os métodos para o cálculo teórico de cargas atômicas são invariavelmente arbitrários, resultando, em geral, em uma grande variação dos valores numéricos das cargas calculadas segundo diferentes metodologias.

A comparação dos valores absolutos de cargas atômicas calculadas segundo diferentes métodos ainda carece de significados. Contudo, há alguns critérios mínimos desejáveis para que um conjunto de cargas atômicas calculadas teoricamente sejam satisfatórias:

- A soma das cargas de todos os átomos na molécula deve resultar na carga total da molécula;

- Devem refletir a simetria da molécula e serem invariantes com respeito à rotação e translação desta;

- Devem ser consistentes com as eletronegatividades atômicas e possuir uma interpretação física clara, sendo portanto compatíveis com a intuição química;

- Não devem apresentar uma dependência elevada com o conjunto de base e devem tender a um limite bem definido quando o conjunto de base tende a completeza;

- Devem reproduzir satisfatoriamente o momento dipolar e o potencial eletrostático molecular. 
Uma maneira inicilamente utilizada para obter essas cargas é através da análise populacional de Mulliken [67, que é seguramente o método mais tradicional e de uso mais difundido entre os químicos. Esse método baseia-se na teoria dos orbitais moleculares, $\left\{\Psi_{i}\right\}$ é definido por uma combinação linear de $k$ orbitais atômicos, também chamados funções de base, $\left\{\chi_{j}\right\}$, cujos os coeficientes são determinados pelo método Hartree-Fock,

$$
\Psi_{i}=\sum_{j=1}^{k} C_{j i} \chi_{j}
$$

A análise populacional de Mulliken consiste em associar um número de elétrons a um determinado átomo de acordo com o que todos os orbitais atômicos centrados naquele átomo contribuem para a densidade eletrônica [32]. Subtraindo esse valor do número atômico $(\mathrm{Z})$ do átomo em questão, obtém se carga parcial líquida sobre aquele átomo.

A análise populacional de Mulliken deve sua grande popularidade em grande parte a intensiva aplicação dos métodos que empregam a teoria do orbitais moleculares e, consequentemente à facilidade com que pode ser calculada. Além disso todas as variáveis necessárias para efetuar a análise populacional são obtidas diretamente, não sendo necessário nenhum custo computacional adicional.

O problema com a análise populacional de Mulliken é que ela não é a única maneira de se fazer análise populacional baseada em orbitais atômicos e também apresenta uma forte dependência do conjunto de base empregada no cálculo [68. Este efeito é geralmente mais pronunciado quando o conjunto de base possue orbitais difusos. Neste caso, a carga originalmente pertencente a um orbital de um determinado átomo pode contribuir para a carga total de um átomo vizinho aumentado artificialmente esta última.

Com o intuito de superar as dificuldades associadas à análise populacional de 
Mulliken, foi proposta a definição de um modelo de cargas pontuais bastante distinto desse último. Esse modelo baseia-se no cálculo de um conjunto de cargas atômicas pontuais de modo que estas representem o melhor possível o potencial eletrostático quântico em um conjunto de pontos pré definidos ao redor da molécula. Estas cargas atômicas são chamadas derivadas do potencial [69|.

Há vários métodos que fazem uso dessa abordagem [70,71], em particular, o método CHELPG (Charges from Electrostatic Potential Grid based) |72 tem sido bastante usado. O método consiste no seguinte procedimento: (i) Cria-se uma grade retangular de pontos em torno da molécula. Dessa grade são excluídos todos os pontos que estão dentro das esferas de van der Walls centradas nos átomos das moléculas; (ii) Calcula-se o potencial eletrostático em cada um dos pontos dessa grade; (iii) Ajustamse as cargas parciais nas posições dos núcleos atômicos de modo a reproduzir o potencial eletrostático. O único vínculo adicional no ajuste é que a carga total tem que ser igual a carga líquida da molécula que gerou o potencial.

As cargas derivadas do potencial apresentam a vantagem de, em geral, serem fisicamente mais satisfatórias que as cargas de Mulliken [73]. Como consequência do seu método de cálculo, as cargas derivadas do potencial reproduzem adequadamente o potencial eletrostático molecular, o que é relevante em simulações de interações intermoleculares, obtendo-se uma descrição adequada de interações eletrostáticas entre moléculas. Em geral, o método CHELPG permite a reprodução satisfatória do momento dipolar. Por conta disso esse método será utilizado neste trabalho para gerar as cargas parciais que serão utilizadas na avaliação da interação intermolecular através de simulações com Monte Carlo. 


\subsection{Modelo Contínuo para o Solvente}

Nos últimos anos tratamentos totalmente quanto-mecânico da interação solutosolvente e solvente-solvente, onde moléculas do solvente interagem explicitamente com o soluto, tem sido introduzido e seu uso vem crescendo rapidamente. Entretanto, isto não elimina a utilidade dos modelos contínuos, cuja maior vantagem é o custo computacional mais baixo. Estes modelos tiveram origem nos trabalhos de Born 74,, Kirkwood 75$]$ e Onsager [76]. No modelo contínuo o solvente é tratado como um material dielétrico, caracterizado por parâmetros macroscópicos, principalmente a constante dielétrica $\epsilon$. Todos esses modelos são baseados na equação de Poisson, que relaciona a distribuição de cargas $\rho$, o potencial eletrostático $\phi$ e a constante dielétrica $\epsilon$ :

$$
\nabla^{2} \phi(r)=-\frac{4 \pi \rho(r)}{\epsilon}
$$

No modelo contínuo, o soluto é colocado em uma cavidade $(\epsilon=1)$ circundada por um dielétrico de constante $\epsilon$. Portanto, há duas regiões distintas para as quais a equação de Poisson deve ser resolvida satisfazendo as condições de contorno usuais na superfície da cavidade.

Onsager, introduziu a idéia de campo de reação. Um dielétrico com cavidade esférica de raio $a$, na qual se encontra uma molécula cuja distribuição de cargas é representada pelo momento de dipolo $\mu$, é polarizado pelo campo elétrico desse dipolo. A distribuição de cargas no dielétrico resultante dessa polarização dá origem ao campo de reação,

$$
R=\frac{2(\epsilon-1)}{2 \epsilon+1} \frac{\vec{\mu}}{a^{3}}
$$

Esse campo reativo é sempre paralelo ao momento de dipolo e, portanto, atuará no soluto aumentando a assimetria na sua distribuição de cargas. Se a molécula for apolar, então não há campo de reação e o campo elétrico sentido pelo soluto deve 
ser bastante reduzido. A equação (2.116 é uma aproximação que considera apenas o primeiro momento da distribuição de cargas. Implementações mais sofisticadas podem utilizar também campos de reação gerados por quadrupolos, octopolos, etc [77].

Um dos pontos a favor do modelo contínuo é a possibilidade em se fazer um tratamento puramente quântico da interação soluto-solvente, pois tratam do solvente sem aumentar o número de elétrons explícitos. Assim, o potencial de interação contém um termo adicional que depende do campo de reação. Incorporando esse termo no hamiltoniano e usando a aproximação de Hartree-Fock, obtém-se:

$$
\left\{F_{i}-\left[\frac{2(\epsilon-1)}{2 \epsilon+1}\right] \frac{1}{a^{3}}\langle\Psi|\mu| \Psi\rangle\right\} \chi_{i}=e_{i} \chi_{i}
$$

sendo $\chi_{i}$ orbitais moleculares. Há um termo na equação (2.117) que depende do momento de dipolo do soluto (e possivelmente de outros momentos), que por sua vez depende da função de onda total $\Psi$, criando um ciclo adicional de autoconsistência. Por isso, tal procedimento é comumente referido por Campo de Reação Autoconsistente (SCRF).

Outro ponto favorável aos modelos contínuos é que a perturbação provocada pelo solvente, a longa distâncias, pode ser bem representada por uma distribuição de cargas, entretanto, para a estrutura microscópica do solvente nas proximidades do soluto, essa distribuição determinada por parâmetros macroscópicos é um simplificação exagerada não sendo possível obter, através desse modelo, uma descrição adequada das ligações de hidrogênio, por exemplo. Além disso, os resultados obtidos com os modelos contínuos apresentam uma dependência com o tamanho da cavidade, o que pode ser entendido olhando-se a expressão do campo de reação, equação (2.116), em que o campo depende inversamente do cubo do raio da cavidade, sendo assim bastante sensível ao mesmo.

A forma da cavidade também é um problema pois poucas moléculas têm uma forma que possa ser considerada esférica ou elipsoidal. Isto pode ser resolvido fazendo com que a cavidade seja formada pela união de esferas centradas nos átomos do soluto. 
Dessa forma, a cavidade tem necessariamente o formato da molécula de referência, sendo que o tamanho das esferas deve estar sujeito a algum tipo de ajuste.

O método mais popular dentre aqueles que utilizam esse tipo de cavidade é o modelo contínuo polarizável (PCM), introduzido por Tomasi e colaboradores [78,79. Eles utilizam os raios de van der Waals acrescidos de aproximadamente $20 \%$ para desenhar a cavidade. Esse modelo tem sido bastante utilizado e com relativo sucesso, principalmente para análise de propriedades que dependam pouco de interações específicas entre soluto e solvente. Esse modelo é o que será considerado aqui quando tratarmos o solvente como um meio contínuo. 


\section{Capítulo 3}

\section{Mecânica Molecular}

\subsection{Introdução}

A utilização de métodos específicos para estudar sistemas líquidos está associada à complexidade das interações intermoleculares e à ausência de simetria espacial que caracterizam esses sistemas. Nesse âmbito, os modelos moleculares para o estudo de líquidos com simulação molecular podem levar em conta características e propriedades das moléculas que constituem o sistema, e o modo como estas interagem. A metodologia de mecânica estatística é, então, utilizada para reproduzir as relações entre interações intermoleculares e propriedades termodinâmicas.

$\mathrm{Na}$ mecânica estatística de equilíbrio todas as propriedades estruturais e termodinâmicas de um sistema de partículas podem, em princípio, ser determinadas se for conhecido o potencial de interação entre as partículas. Assumindo então que o potencial de interação $U(r)$ é conhecido para todas as partículas, temos que o hamiltoniano $\mathcal{H}$ para um sistema de $\mathrm{N}$ partículas será dado por [26]:

$$
\mathcal{H}=\mathcal{H}(\mathbf{r}, \mathbf{p})=\sum_{i=1}^{N} \frac{p_{i}^{2}}{2 m_{i}}+U(r)
$$

onde o primeiro termo é a energia cinética do sistema e o segundo termo é o potencial de interação, $\mathbf{r}$ é a coordenada coletiva representando todas as partículas, $\mathbf{r}=\left(\mathbf{r}_{1}, \mathbf{r}_{2}, \ldots, \mathbf{r}_{\mathbf{N}}\right)$ 
e $\mathbf{p}$ é o conjunto dos momentos lineares de todas as partículas.

Assumindo que o sistema esteja em contato com um reservatório térmico infinitamente grande, com temperatura absoluta $T$ e que o número de partículas $N$ e o volume $V$ são conhecidos (ensemble $N V T$ [80]), a grandeza que faz a conexão entre o mundo microscópico (mecânica estatística) e o macroscópico (termodinâmica), no limite termodinâmico, é a função canônica de partição $Z$, esta função raramente pode ser obtida exatamente, exceto para sistemas ou problemas-modelo simples.

A função de partição é definida por:

$$
Z(N, V, T)=\iint e^{-\beta \mathcal{H}(\mathbf{r}, \mathbf{p})} d \mathbf{p} d \mathbf{r}
$$

onde $\mathcal{H}$ é o hamiltoniano dado pela equação (3.1) e $\beta=1 / k_{B} T, k_{B}$ é a constante de Boltzmann $\left(k_{B}=1,38 \cdot 10^{23} \mathrm{~J} / K\right)$.

Assim como o hamiltoniano, a função de partição $Z$ também é separável nas suas partes cinética e potencial, também chamada configuracional,

$$
\begin{aligned}
Z(N, V, T) & =Z_{K} \cdot Z_{\text {conf }} \\
Z_{K} & =\int \exp \left(-\beta \sum_{i=1}^{N} \frac{p_{i}^{2}}{2 m_{i}}\right) d \mathbf{p} \\
Z_{\text {conf }} & =\int \exp (-\beta U(\mathbf{r})) d \mathbf{r}
\end{aligned}
$$

No formalismo da mecânica estatística 81,82 , uma propriedade termodinâmica observável é uma média no ensemble de estados microscópicos de um sistema, e a escolha apropriada de um ensemble está associada aos vínculos que são impostos ao sistema em estudo. Os sistemas comumente estudados em simulação de líquidos estão sob as condições do ensemble canônico $(N V T)$ e do ensemble isobárico-isotérmico $(N P T)$, em que o número de partículas $N$, a pressão $P$ e a temperatura $T$ são mantidos 
fixos. O valor médio de uma grandeza $W(\mathbf{r}, \mathbf{p})$ no ensemble $N V T$, por exemplo, é dada por:

$$
\langle W\rangle_{N V T}=\frac{1}{Z} \int W(\mathbf{r}) e^{-\beta \mathcal{H}(\mathbf{r}, \mathbf{p})} d \mathbf{r} d \mathbf{p}
$$

onde a média é feita em cima do ensemble de configurações.

Neste trabalho as simulações clássicas foram realizadas no sentido de gerar configurações acessíveis ao sistema solvatado (Soluto + Solvente). Cada configuração possui informações acerca das coordenadas de todas as moléculas. Um ponto importante na geração dessas configurações é a definição dos potenciais intermoleculares que descrevem a interação soluto-solvente, sendo estes cruciais para uma adequada descrição das relações entre propriedades moleculares e características observadas experimentalmente. Há duas maneiras de se obter as configurações acessíveis. Uma delas é através do método de Dinâmica Molecular (DM) [83|. Nesse método, a partir do potencial de interação $U(\mathbf{r})$, as forças que atuam sobre os átomos são calculadas, $\mathbf{F}=-d \mathbf{U} / d \mathbf{r}$, e as equações de movimento são resolvidas para um intervalo de tempo, $\delta t$. Assim as configurações do sistema são geradas de forma determinística. A outra maneira é através do método Monte Carlo (MC) em que as configurações são geradas por processos estocásticos e não há como determinar a ordem temporal dessas configurações geradas.

Neste trabalho as configurações foram geradas através do método Monte Carlo, pois não havia interesse em descrever propriedades dinâmicas do sistema. O seu uso reduz considerávelmente o custo computacional quando comparado com a Dinâmica Molecular. Vale salientar que todo desenvolvimento teórico feito sobre as simulações computacionais é baseado na hipótese de que tanto o processo determinístico quanto o estocástico, para gerar as configurações, são ergódicos 84 . 


\subsection{Método Monte Carlo}

O método Monte Carlo foi desenvolvido por von Neumann, Ulam e Metropoplis para estudar a difusão de nêutrons em materiais que poderiam sofre fusão. Porém, desde o início do século XIX já se fazia uso do método de amostragem para estudar alguns problemas. Esses métodos de amostragem envolvem a geração de números randômicos que depois são usados para uma série de operações aritméticas e lógicas, que serão sempre as mesmas a cada passo da simulação. Essas operações são realizadas com sucesso pelos computadores e portanto, o desenvolvimento da técnica está intimamente ligada ao desenvolvimento dessas máquinas.

O método Monte Carlo é comumente usado para calcular numericamente as integrais envolvidas na equação (3.15), aproximando-as por somas sobre um número $n$ de pontos escolhidos aleatoriamente no espaço de fase, de modo que podemos escrever,

$$
\langle W\rangle=\frac{\sum_{n=1}^{N} W(\mathbf{r}) e^{-\beta U(\mathbf{r})}}{\sum_{n=1}^{N} e^{-\beta U(\mathbf{r})}}
$$

Entretanto, esse método de amostragem aleatória não é muito útil para grande maioria dos problemas de interesse em que o número $n$ de observações é muito grande no espaço multiconfiguracional. Ainda, para N moléculas na densidade do líquido, uma amostragem aleatória dos estados levaria a grande proximidade ou mesmo superposição de duas moléculas, essas configurações, por sua vez, resultam em uma energia alta do sistema e, portanto, uma probabilidade baixa do sistema. Estas configurações devem ser evitadas.

Um procedimento mais eficiente para a seleção das configurações é restrito às regiões mais densas do espaço de fase e termodinamicamente mais prováveis. O algoritmo de amostragem modificado para o método MC convencional, proposto por Metropolis e colaboradores [85], é denominado importance sampling e consiste em evitar a amostragem excessiva de configurações que tem pequena contribuições para o 
cálculo da média.

Na proposta de Metropolis a idéia é amostrar configurações a partir de uma distribuição $\rho(r)$ transformando a soma da equação $(3.7)$ em

$$
\langle W\rangle=\frac{\frac{1}{\rho(r)} \sum_{n=1}^{N} W(\mathbf{r}) e^{-\beta U(\mathbf{r})}}{\frac{1}{\rho(r)} \sum_{n=1}^{N} e^{-\beta U(\mathbf{r})}}
$$

que representa a soma com um peso dado pelo termo $\rho(r)$, onde $\rho(r)$ pode ser qualquer gerando uma distribuição preferencial. No MC Metropolis esta seleção é feita levando em conta os fatores de Boltzamann, $\rho(r)=\rho(r)_{N V T}=e^{-\beta U(r)}$. Usando esta distribuição de probabilidades na equação (3.8), obtemos:

$$
\langle W\rangle_{N V T}=\frac{1}{N} \sum_{n=1}^{N} W(r(n))
$$

onde $r(n)$ é o conjunto de coordenadas $r=\left(r_{1}^{n}, r_{2}^{n}, r_{3}^{n}, \ldots\right)$ da configuração específica $n$.

Na equação (3.9) $W(r(n))$ é o valor de $W(r)$ nas configurações geradas pela simulação. Como as configurações são geradas com o peso de Boltzmann a média $\langle W\rangle$ é uma média simples das grandezas $W(r)$. Os estados que não são estatisticamente representativos são filtrados pela amostragem de Metropolis.

No algoritmo de Metropolis, a transição de uma configuração $\Gamma_{i}$ para uma configuração $\Gamma_{i+1}$ é obtida através de um processo probabilístico denominado cadeia de Markov [83], que é construida de tal maneira que esta cadeia tenha uma distribuição limite igual a função de partição ou a função de distribuição desejada. Uma cadeia de Markov é uma sequencia de tentativas que deve satisfazer duas condições:

1. O resultado de cada tentativa deve pertencer a um conjuto finito de resultados, $\left\{\Gamma_{1}, \Gamma_{2}, \Gamma_{3}, \ldots\right\}$, chamado de espaço de estados. 
2. O resultado de cada tentativa deve depender somente do resultado da tentativa imediatamente anterior.

Dois estados $\Gamma_{m}$ e $\Gamma_{n}$ são ligados por uma taxa de transição $\pi_{m n}$, que é a probabilidade para o sistema sair do estado $m$ para o estado $n$. As probabilidades de transição formam uma matriz que é chamada matriz de transição, essa matriz é irredutível, isto é, a partir de um estado é possível acessar todos os outros estados do sistema. Isto também quer dizer que a cadeia é ergódica.

Portanto, seja $\rho_{m}$ a distribuição de probabilidade do estado $\Gamma_{m}$ através da matriz de transição $\pi_{m n}$ podemos obter $\rho_{n}$, que é a distribuição para o estado $\Gamma_{n}$. A matriz de transição deve obdecer a seguinte equação de autovalores com autovalor 1 ,

$$
\rho_{n}=\sum_{m} \rho_{m} \pi_{m n} \quad(m \neq n)
$$

$\pi$ é uma matriz estocástica desde que:

$$
\pi_{m n} \geq 0 \quad \text { e } \quad \sum_{n} \pi_{m n}=1
$$

essa é uma matriz de transição para uma cadeia de Markov.

No caso de um sistema líquido, é preciso construir uma matriz de transição muito grande, que seja estocástica e ergódica. Nesse caso os elementos dessa matriz são desconhecidos, mas os elementos do vetor da distribuição limite da cadeia são dados pelos valores da função de partição em cada estado do sistema, ou seja, $\rho_{m}=\rho_{N V T}\left(\Gamma_{m}\right)$ para cada estado $\Gamma_{m}$.

Uma técnica útil para resolver a equação (3.10 é substituir essa equação por uma condição desnecessária, porém forte, chamada de reversibilidade microscópica 
(ou balanço detalhado):

$$
\rho_{m} \pi_{m n}=\rho_{n} \pi_{n m}
$$

Nessa condição de reversibilidade microscópica, os dois estados estão ligados por uma taxa de transição, onde a probabilidade do sistema sair do estado $m$ e ir para o estado $n$ é a mesma para o sistema sair do estado $n$ e ir para o estado $m$.

Agora, somando sobre todos os estados $m$ na equação 3.12 e usando a equação (3.11) nós podemos reescrever a equação (3.10,

$$
\begin{aligned}
\sum_{m} \rho_{m} \pi_{m n} & =\sum_{m} \rho_{n} \pi_{n m} \\
& =\rho_{n} \sum_{m} \pi_{n m} \\
& =\rho_{n}
\end{aligned}
$$

Uma maneira adequada para construir uma trajetória no espaço de fase usando o ensemble canônico envolve a escolha de uma matriz de transição que satisfaça as condições de ser estocástica, equação (3.11), e a reversibilidade microscópica, equação (3.12). O primeiro arranjo feito desse modo foi sugerido por Metropolis e colaboradores em 1953 85 e é comumente conhecido como solução assimétrica. Se temos dois estados $m$ e $n$ com probabilidades $\rho_{m}$ e $\rho_{n}$, respectivamente, esta solução assimétrica considera dois casos:

$$
\begin{array}{lll}
\pi_{m n}=\alpha_{m n} & \rho_{n} \geq \rho_{m} & m \neq n \\
\pi_{m n}=\alpha_{m n}\left(\rho_{n} / \rho_{m}\right) & \rho_{n}<\rho_{m} & m \neq n
\end{array}
$$

onde para o ensemble canônico, $\rho_{n}=\rho_{N V T}\left(\Gamma_{n}\right)=e^{-\beta U\left(r_{n}\right)}$ e $\rho_{m}=\rho_{N V T}\left(\Gamma_{m}\right)=$ 
$e^{-\beta U\left(r_{m}\right)}$. Sendo também importante permitir a possibilidade de que o líquido permaneça no mesmo estado, ou seja,

$$
\pi_{m m}=1-\sum_{n \neq m} \pi_{m n}
$$

Na solução proposta por Metropolis, $\alpha$ é uma matriz estocástica simétrica, $\left(\alpha_{m n}=\alpha_{n m}\right)$, e essa simetria garante que a solução obedeça à reversibilidade microscópica. Para implementar a solução de Metropolis na matriz de transição é preciso especificar a matriz $\alpha$. Essa matriz é responsável por levar o sistema do estado $m$ para qualquer um dos estados vizinhos $n$ com a mesma probabilidade. Existe muita liberdade na escolha da matriz $\alpha$ e a única restrição é de que esta seja simétrica.

Na prática, para um simulação no ensemble $N V T$, temos que se uma molécula é movida para uma nova posição e a energia do sistema diminui, $\left(U_{F}<U_{I}\right)$ então esta nova posição é aceita; porém se a nova posição provoca um aumento na energia $\left(U_{F}>U_{I}\right)$ então um número aleatório $\gamma$ entre 0 e 1 é gerado e se este número for menor ou igual à probabilidade de transição $\pi_{F, I}=\rho_{F} / \rho_{I}=e^{-\left(U_{F}-U_{I}\right) / k_{B} T}=e^{-\Delta U / k_{B} T}$, a nova posição é aceita, apesar da energia ter aumentado, caso contrário $\left(\gamma>e^{-\Delta U / k_{B} T}\right)$ a nova posição é rejeitada.

Esta solução proposta por Metropolis não é única, porém é uma forma muito simples de gerar uma cadeia de Markov.

Para tratarmos a proposta de Metropolis no ensemble isotérmico-isobárico, $(N P T)$, devemos apenas usar a distribuição de probabilidades que caracterizam esse ensemble, ou seja, $\rho_{m}=\rho_{N P T}\left(\Gamma_{m}\right)$ onde

$$
\rho_{N p T}(r)=e^{-\beta[U(r)+p V]}
$$

Esse ensemble foi pensado como sendo particularmente apropriado para simular misturas já que medidas experimentais são feitas à pressão constante e teorias de misturas 
são sempre formuladas com essa suposição. O cálculo da média de uma grandeza usando o ensemble NPT será dado por:

$$
\langle W\rangle_{N P T}=\frac{\int W(\mathbf{r}) e^{-\beta[U(\mathbf{r})+P V]} d \mathbf{r} d V}{\int e^{-\beta[U(\mathbf{r})+P V]}}
$$

A dificuldade nesta expressão é que o volume além de está explícito na probabilidade do ensemble, está também presente implicitamente no domínio de integração das coordenadas das partículas, tornando difícil o seu controle. Sendo assim, é mais interessante desvincular o domínio de $\mathrm{U}(\mathbf{r})$ do volume [86]. Isto é feito tornando adimensionais as coordenadas das partículas do sistema, através da transformação:

$$
\mathbf{r}_{i} \rightarrow\left(L_{x} q_{i x}, L_{y} q_{i y}, L_{z} q_{i z}\right)
$$

onde $\mathbf{r}_{i}$ representa as coordenadas da partícula $i$ e $\mathbf{q}_{i}=\left(q_{i x}, q_{i y}, q_{i z}\right)$. O domínio da coordenada $q_{i j}$, com $i=1,2,3, \ldots, N$ e $j=x, y, z$, é o intervalo [0,1], onde esse intervalo vai depender das coordenadas dos vértices da caixa que contém o volume. Com essa transformação a equação 3.20 passa a ser escrita como:

$$
\langle W\rangle_{N P T}=\frac{\int W(\mathbf{Q}) e^{-\beta[U(\mathbf{Q})+P V]} V^{N} d \mathbf{Q} d V}{\int e^{-\beta[U(\mathbf{Q})+P V]}}
$$

$\operatorname{com} \mathbf{Q}=\left(\mathbf{q}_{1}, \mathbf{q}_{2}, \ldots, \mathbf{q}_{N}\right)$. A distribuição de probabilidades na equação 3.19 pode ser reescrita como:

$$
\rho_{N p T}(r)=e^{-\beta[U(Q)+P V]+N \ln V}
$$

Portanto, substituindo a densidade de probabilidade $\rho_{N P T}$ dada pela equação (3.23) no conjunto de equações (3.17) e (3.18) nós teremos uma solução analoga à proposta de Metropolis para o ensemble $N V T$. A mudança na forma da distribuição de probabilidade para o ensemble NPT é interessante porque torna a variação no 
volume independente da mudança nas coordenadas do sistema que são relativas ao volume corrente da simulação.

\subsubsection{Detalhes da Implementação}

Em nosso trabalho, o estudo do sistema solvatado foi feito usando o método Monte Carlo tal como implementado no programa DICE [87] cuja implementação segue da seguinte forma :

1. Iniciamos a simulação com uma configuração inicial qualquer de um sistema com $\mathrm{N}$ moléculas confinadas em uma caixa de volume $\mathrm{V}$ a uma temperatura T. A partir daí replica-se a caixa em todas as direções, impondo condições de contorno periódicas, para eliminar os efeitos de borda. Este método consiste em replicar a caixa com o sistema original em todas as direções, fazendo com que as moléculas da caixa original não interajam mais com as paredes, mas sim com outras moléculas que são réplicas das existentes na caixa original. Há também a introdução de um raio de corte $r_{c}$, significando que cada molécula só interage com outras que estão separadas por uma distância menor que o raio de corte.

2. Uma nova configuração é obtida partindo da configuração inicial, isso ocorre através de uma translação de $\delta_{r}=\left(\delta_{x}, \delta_{y}, \delta_{z}\right)$ e rotação de $\delta \Theta$, num eixo sorteado, de alguma molécula sorteada. A translação é feita calculando-se um novo valor para as coordenadas $(x, y, z)$ do centro de massa da molécula através das equações:

$$
\begin{aligned}
& x_{i+1}=x_{i}+a \gamma_{1} \\
& y_{i+1}=y_{i}+a \gamma_{2} \\
& z_{i+1}=z_{i}+a \gamma_{3}
\end{aligned}
$$


onde $\gamma_{i}$ é um número aleatório entre -1 e 1 e $a$ o deslocamento máximo, que pode ser ajustado ao longo da simulação.

A rotação é feita sorteando-se um eixo aleatório $\gamma_{4}$ dentre os eixos $x, y, z$ e um ângulo para rotacionar a molécula através da equação:

$$
\phi_{i+1}=\phi_{i}+b \gamma_{5}
$$

sendo $b$ o ângulo máximo de rotação.

Para o tratamento no ensemble NPT há também a mudança no volume, e esta mudança se dá obedecendo as seguintes equações:

$$
\begin{aligned}
& L_{i+1, x}=L_{i x}+c \gamma_{6} \\
& L_{i+1, y}=L_{i y}+c \gamma_{6} \\
& L_{i+1, z}=L_{i z}+c \gamma_{6}
\end{aligned}
$$

sendo $c$ a variação máxima dos lados da caixa, este parâmetro também pode ser ajustado durante a simulação.

Este movimento de translação e rotação é feito para todas as moléculas do sistema e em cada uma é aplicada o teste de aceitação.

3. Nem todos os movimentos moleculares são aceitos. É a técnica de amostragem que estabelece as regras de aceitação, ou rejeição dos movimentos. Neste programa é usada a técnica de amostragem de Metropolis, que foi detalhada na seção 3.2, essa técnica usa a variação de energia entre as configurações como termo decisivo na regra de aceitação de uma nova configuração.

4. Para o cálculo das propriedades termodinâmicas são usadas médias e flutuações de algumas grandezas obtidas durante a simulação, são elas: a energia $U$, o 
primeiro virial $W=-1(1 / 3) r(\partial U / \partial r)$ e o segundo virial $\Theta=(1 / 9) r(\partial(r \partial U / \partial r) / \partial r)$; acumula-se esses valores para a média e volta-se para a etapa 2 do processo.

Cada vez que se chega a este ponto, dizemos que foi realizado um passo de Monte Carlo.

Ao final da simulação, há uma cadeia de configurações que descrevem a evolução do sistema. Em geral, esta evolução pode ser dividida em dois estágios. O primeiro é um estágio não estacionário, termalização, e o segundo é um estágio estacionário, conhecido como estágio de equilíbrio ou estágio de médias. Estes dois estágios podem ser facilmente observados no comportamento da energia do sistema durante a simulação, pois o sistema sai da energia inicial $U_{0}$ e gradualmente atinge um valor de energia médio $\langle U\rangle$, onde fica flutuando. Para o cálculo das propriedades estruturais e termodinâmicas somente as configurações geradas no equilíbrio são consideradas.

\subsubsection{Potencial de Interação Intermolecular}

Uma boa descrição de um sistema molecular está intimamente ligada a escolha do potencial de interação $U(\mathbf{r})$, esse potencial pode ser tratado como uma soma da energia intramolecular de cada molécula e a energia intermolecular. Para modelos de moléculas rígidas, onde a distância e os ângulos das ligações são mantidos fixos, a energia interna é constante durante todo o processo de geração das configurações. Portanto, a representação usada para a energia de uma configuração, nesses sistemas, consiste somente da contribuição intermolecular desprezando-se assim as contribuições intramoleculares devido a rotações e vibrações internas da molécula.

O potencial intermolecular pode ser escrito como:

$$
U(\mathbf{r})=\sum_{i} u_{1}\left(\mathbf{r}_{i}\right)+\sum_{i} \sum_{j>i} u_{2}\left(\mathbf{r}_{i}, \mathbf{r}_{j}\right)+\sum_{i} \sum_{j>i} \sum_{k>j} u_{3}\left(\mathbf{r}_{i}, \mathbf{r}_{j}, \mathbf{r}_{k}\right)+\ldots
$$


sendo $u_{1}$ o potencial que atua em um corpo e representa o efeito sobre o sistema de forças externas; o segundo termo, $u_{2}$, é o potencial de pares e representa a interação entre dois corpos, $u_{2}=u_{2}\left(r_{i j}\right)$ onde $r_{i j}=\left|\mathbf{r}_{i}-\mathbf{r}_{j}\right|$; o terceiro termo, $u_{3}$, representa a interação entre três corpos; o termo seguinte irá representar a interação entre quatro corpos e assim sucessivamente. O que se observa é que, em geral, os termos de interação entre quatro corpos e ordens seguintes são pequenos comparados aos termos $u_{2}$ e $u_{3}$, potanto, o potencial de interação é truncado em $u_{3}$. Em muitos sistemas a contribuição de três corpos é relevante [83,89], entretanto para sistemas sem orientação preferêncial, como acontece em geral com os líquidos, esta interação já é bastante pequena de tal forma que, para modelar esses sistemas, o potencial é truncado no segundo termo e o potencial de pares é substituido por um potencial efetivo de pares que inclui a contribuição de muitos corpos.

$$
U(\mathbf{r})=\sum_{i} u_{1}\left(\mathbf{r}_{i}\right)+\sum_{i} \sum_{j>i} u_{2}^{e f f}\left(r_{i j}\right)
$$

uma consequência desta aproximação é que o potencial efetivo de pares tem que reproduzir dados experimentais.Aliás, é através destes dados experimentais e também de resultados de estudos ab initio que se constrói o potencial efetivo.

Considerando sistemas em que não atuam forças externa, $u_{1}=0$, o potencial de interação será dado por:

$$
U(\mathbf{r})=\sum_{i} \sum_{j>i} u_{2}^{e f f}\left(r_{i j}\right)
$$

Em geral, esse potencial efetivo é escrito como uma soma de potenciais de pares entre os sítios das moléculas, esses sítios, em sua maioria, são representados pelos átomos das moléculas. Nesse caso, a interação entre duas moléculas $a$ e $b$ é descrita 
por:

$$
U_{a b}=\sum_{i}^{e m} \sum_{j}^{a m b} U\left(r_{i j}\right)
$$

onde $i$ são os sítios da molécula $a, j$ os sítios da molécula $b$ e $r_{i j}$ a distância entre os sítios $i$ e $j$. Na figura (3.1) ilustramos a interação entre dois sítios de duas moléculas.

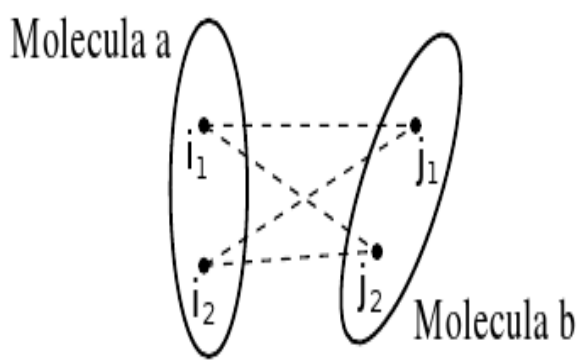

Figura 3.1: Ilustração da interação entre os sítios $i$ da molécula $a$ e os sítios $j$ da molécula $b$. Figura retirada de 88

A energia potencial intermolecular é então reduzida a uma grande soma de potenciais de interação de pares sítio-sítio. Como não se conhece a expressão formal para $U\left(r_{i j}\right)$, há várias maneiras de escrevê-lo analiticamente. O potencial, $U\left(r_{i j}\right)$, mais usado para tratar sistemas líquidos é o potencial de Lennard-Jones [90] com um termo adicional coulombiano, equação (??).

$$
U\left(r_{i j}\right)=4 \epsilon_{i j}\left[\left(\frac{\sigma_{i j}}{r_{i j}}\right)^{12}-\left(\frac{\sigma_{i j}}{r_{i j}}\right)^{6}\right]+\frac{q_{i} q_{j}}{r_{i j}}
$$

sendo o primeiro termo o potencial de Lennard-Jones. Aqui, cada sítio é representado por dois parâmetros $\left(\epsilon_{i}\right.$ e $\left.\sigma_{i}\right)$ e uma carga $q_{i}$. Na equação $3.31, \epsilon_{i j}=\left(\epsilon_{i} \epsilon_{j}\right)^{1 / 2}$ e $\sigma_{i j}=\left(\sigma_{i} \sigma_{j}\right)^{1 / 2}$. A carga $q_{i}$ é responsável pela descrição dos termos eletrostáticos. Os parâmetros $\epsilon$ e $\sigma$ podem ser interpretados como sendo a energia de ligação e a distância entre os sítios, respectivamente. 


\subsubsection{Propriedades Estruturais: Função de Distribuição Radial}

Para estudarmos as propriedades estruturais, comumente é usada a função de distribuição radial (RDF) de pares, $G(r)$ [83]. Experimental, as funções de distribuição radial podem ser determinadas a partir de técnicas de difração de raios-X, espalhamentos de neutrons e difração de elétrons . Esta função nos dá a probabilidade de encontrarmos um par de átomos a uma distância $r$, relativa a probabilidade esperada para um sistema de mesma densidade e uma distribuição totalmente aleatória, conhecida como distribuição de gás ideal.

Para um sistema de átomos idênticos a RDF deve ser calculada como uma média sobre os pares de átomos indistinguíveis. Numa simulação, a RDF entre átomos de tipo $i$ e átomos do tipo $j, G_{i j}$, é calculada através do histograma de distâncias dos pares de átomos $i$ e $j$ :

$$
G_{i j}\left(r+\frac{1}{2} d r\right)=\frac{n_{i j}(r, r+d r)}{n^{i d}(r, r+d r)}
$$

sendo $n_{i j}(r, r+d r)$ o número de pares $i j$ que estão separados por uma distância entre $r$ e $r+d r$ e $n^{i d}$ é o número de pares equivalente num gás ideal de mesma densidade,

$$
n^{i d}(r, r+d r)=\frac{4 \pi}{3} \rho\left[(r+d r)^{3}-r^{3}\right]
$$

Na figura (3.2) ilustramos a estrutura de um líquido atômico e a representação de sua estrutura pela função de distribuição radial. Os picos da figura (3.2) definem as camadas de solvatação.

Integrando $G_{i j}(r)$ numa casca esférica, podemos analisar como o número de moléculas se distribuem radialmente em torno de outra,

$$
N_{s}(r)=4 \pi \frac{N}{V} \int_{0}^{r} G_{i j}(r) r^{2} d r
$$


O número de moléculas que resulta da integração do primeiro pico é conhecido como número de coordenação e é utilizado para definir a primeira camada de solvatação. A segunda e terceira camada estão associadas as integrações do segundo e terceiro pico da Gij, respectivamente.

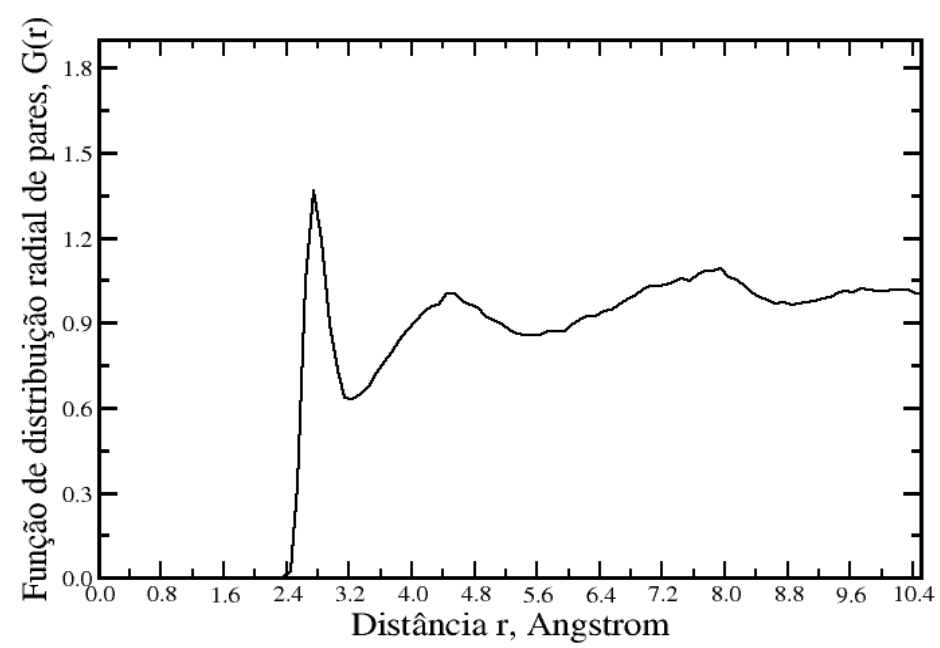

Figura 3.2: Ilustração da estrutura de um líquido atômico e a representação dos picos da $G(r)$ correspondente. Isômero anti da molécula OM.

\subsubsection{Correlação Estatística com Função de Autocorrelação}

Em qualquer estudo utilizando simulações computacionais para representar propriedades que são médias derivadas da mecânica estatística, é essencial garantir a convergência. Para isto, existem duas quantidades que podem ser utilizadas para analisar a eficiência de uma simulação: o tempo de correlação $\tau$ e a ineficiência estatística $s$. Estas duas quantidades são calculadas de formas independentes, mas estão relacionadas 83 por:

$$
s \approx 2 \tau
$$

O tempo ou intervalo de correlação $\tau$ é definido como a integral da função de 
autocorrelação temporal, $C(t)$ :

$$
\tau=\int_{0}^{\infty} C(t) d t
$$

onde num contexto de Monte Carlo, o tempo não está envolvido, essa variável é trocada pelo intervalo de passos de MC. Nesse contexto, a correlação entre as configurações é representada pela autocorrelação da energia, assim, $C(t)$ é escrita como:

$$
C(t)=\frac{\left\langle\delta E_{i} \delta E_{i+t}\right\rangle}{\left\langle\delta E^{2}\right\rangle}=\frac{\sum_{i}\left(E_{i}-\langle E\rangle\right)\left(E_{i+n}-\langle E\rangle\right)}{\sum_{i}\left(E_{i}-\langle E\rangle\right)^{2}}
$$

onde $E_{i}$ é a energia de uma configuração $i$ e $E_{i+t}$ é a energia da configuração gerada após $t$ passos MC.

Em MC, devido a natureza markoviana dos pontos que geram $C(t)$, essa função apresenta um comportamento exponencial [88, 91] do tipo:

$$
C(t)=\sum_{i}^{n} c_{i} e^{-t \backslash \tau_{i}} \quad\left(\tau_{1}>\tau_{2}>\ldots>\tau_{n}>0\right)
$$

onde $\tau_{i}$ representa os tempos de correlação caracteristicos do sistema. Na prática, podemos escrever essa função como sendo composta por somente duas exponenciais

$$
C(t)=c_{1} e^{-t \backslash \tau_{1}}+c_{2} e^{-t \backslash \tau_{2}}
$$

onde em geral, $\tau_{1} \gg \tau_{2}$. Substituindo $C(t)$ dado pela equação 3.39$)$ na equação 3.36$)$ nós obtemos que o tempo de correlação é dado por:

$$
\tau=c_{1} \tau_{1}+c_{2} \tau_{2}
$$

Um exemplo desse comportamento é mostrado na figura (3.3). Podemos observar que um decaimento exponencial duplo consegue ajustar bem o comportamento da função de autocorrelação. Nesse exemplo, temos que um intervalo de $t=1000$ entre as configurações nos fornece uma correlação de aproximadamente $14 \%$ 


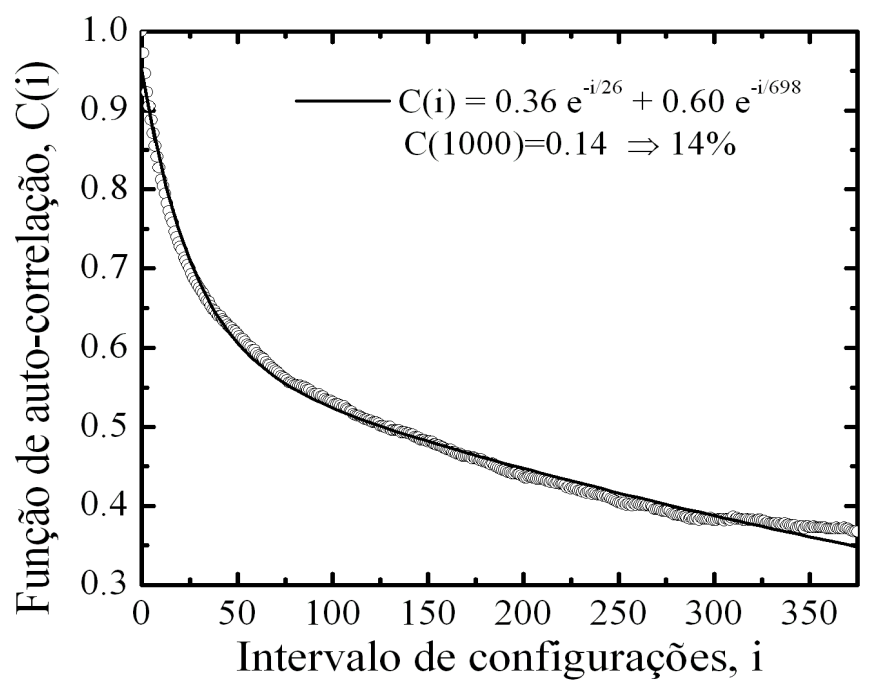

Figura 3.3: Típica função de autocorrelação da energia, calculada para o caso do isômero syn do Óxido Mesitil em água, usando método Monte Carlo. Ensemble NPT condições normais de pressão e temperatura

Rigorosamente, é necessário uma separação infinita $(t \rightarrow \infty)$ para que as configurações estejam descorrelacionadas $(C(t) \approx 0)$. Entretanto, na prática duas configurações serão consideradas descorrelacionadas se estiverem separadas por um intervalo $t=s=2 \tau$, situação em que a correlação está em torno de $13 \%$.

\subsubsection{Energia Livre com Perturbação Termodinâmica}

A energia livre para um sistema de $N$ moléculas, descrito pelo hamiltoniano (3.1) e em contato com um reservatório térmico e de pressões, é dada pela energia livre de Gibbs [80],

$$
\begin{aligned}
G & =-k_{B} T \ln Q \\
Q & =\iint \exp \left[-(U(\mathbf{r})+P V) / k_{B} T\right] d \mathbf{r} d V
\end{aligned}
$$


onde $Q$ é a função de partição no ensemble isobárico-isotérmico $(N P T)$. Esta função de partição é inacessível computacionalmente de forma que o cálculo direto de $G$ é impraticável, por outro lado, é possível calcularmos diferenças de energia livre.

Este problema em estimar a energia livre, através de simulação de MC com o método Metropolis, tem sido discutido na literatura $92,93,94]$. Dentre as metodologias existentes, discutiremos aqui a teoria de perturbação termodinâmica, que foi introduzida por Zwazing [95] e que tem sido largamente utilizada [96, 97, 98, 99.

Aqui o método perturbativo é usado para calcular a diferença de energia livre de Gibbs, $\Delta G$, entre dois estados $F$ e $I$, bem definidos, relacionados pela perturbação $\Delta U$ da forma:

$$
U_{F}=U_{I}+\Delta U
$$

onde $U_{I}$ é o potencial intermolecular do sistema não perturbado e $U_{F}$ é o potencial intermolecular do sistema perturbado.

Assim, a diferença de energia livre de Gibbs, $\Delta G$, que acompanha a transformação entre o estado inicial não perturbado $I$ e o estado final $F$, pode ser escrito como:

$$
\Delta G(I \rightarrow F)=G_{F}-G_{I}=-k_{B} T \ln \frac{Q_{F}}{Q_{I}}
$$

Substituindo a expresão para a função de partição dada pela equação (3.42) e usando a relação dada pela equação 3.43 , nós podemos obter a razão $Q_{F} / Q_{I}$, 


$$
\begin{aligned}
\frac{Q_{F}}{Q_{I}} & =\frac{\iint \exp \left[-\left(U_{I}(\mathbf{r})+\Delta U(\mathbf{r})+P V\right) / k_{B} T\right] d \mathbf{r} d V}{\iint \exp \left[-\left(U_{I}(\mathbf{r})+P V\right) / k_{B} T\right] d \mathbf{r} d V} \\
& =\frac{\iint e^{-\Delta U / k_{B} T} \exp \left[-\left(U_{I}(\mathbf{r})+P V\right) / k_{B} T\right] d \mathbf{r} d V}{\iint \exp \left[-\left(U_{I}(\mathbf{r})+P V\right) / k_{B} T\right] d \mathbf{r} d V}
\end{aligned}
$$

Usando definição de valor médio dada pela equação 3.22 , nós vemos que a razão $Q_{F} / Q_{I}$ pode ser reescrita da seguinte forma:

$$
\frac{Q_{F}}{Q_{I}}=\left\langle e^{-\Delta U / k_{B} T}\right\rangle_{I}
$$

$\log$,

$$
\Delta G(I \rightarrow F)=-k_{B} T \ln \frac{Q_{F}}{Q_{I}}=-k_{B} T \ln \left\langle e^{-\Delta U / k_{B} T}\right\rangle_{I}
$$

onde $\left\langle e^{-\Delta U / k_{B} T}\right\rangle_{I}$ indica que a média estatística é feita no ensemble de configurações do estado não perturbado $I$.

Uma vez que somente diferenças de energia livre estão envolvidas, os estados inicial e final podem ser permutados, assim podemos escrever,

$$
\Delta G(F \rightarrow I)=-k_{B} T \ln \frac{Q_{I}}{Q_{F}}=-k_{B} T \ln \left\langle e^{\Delta U / k_{B} T}\right\rangle_{F}
$$

sendo $\left\langle e^{\Delta U / k_{B} T}\right\rangle_{F}$ a média amostrada no ensemble de configurações do estado perturbado.

Estados intermediários entre $I$ e $F$ podem ser definidos fazendo o potencial de interação intermolecular depender de um parâmetro de acoplamento $\lambda$, que toma valores discretos no intervalo $[0,1]$,

$$
U\left(\lambda_{i}\right)=\lambda_{i} U_{F}+\left(1-\lambda_{i}\right) U_{I}
$$


onde $U\left(\lambda_{i}=0\right)=U_{I}$ e $U\left(\lambda_{i}=1\right)=U_{F}$. Portanto, quando $\lambda_{i}$ variar de forma adequada no intervalo [0,1], o sistema inicial, caracterizado por $U_{I}$, é gradualmente transformado no sistema final, caracterizado por $U_{F}$.

O cálculo da variação de energia livre entre o subintervalo $\lambda_{i}$ e $\lambda_{i+1}$ é obtida usando o mesmo procedimento anterior

$$
\Delta G\left(\lambda_{i} \rightarrow \lambda_{i+1}\right)=-k_{B} T \ln \left\langle e^{-\Delta U\left(\lambda_{i} \rightarrow \lambda_{i+1}\right) / k_{B} T}\right\rangle_{\lambda_{i}}
$$

onde $\Delta U\left(\lambda_{i} \rightarrow \lambda_{i+1}\right)=U\left(\lambda_{i+1}\right)-U\left(\lambda_{i}\right)$. Em sentido oposto, temos:

$$
\Delta G\left(\lambda_{i} \rightarrow \lambda_{i-1}\right)=-k_{B} T \ln \left\langle e^{\Delta U\left(\lambda_{i} \rightarrow \lambda_{i-1}\right) / k_{B} T}\right\rangle_{\lambda_{i}}
$$

onde $\Delta U\left(\lambda_{i} \rightarrow \lambda_{i-1}\right)=U\left(\lambda_{i-1}\right)-U\left(\lambda_{i}\right)$. É importante observar também que a média calculada nas equações (3.51) e (3.52) é feita no ensemble de configurações do estado intermediário $\lambda_{i}$.

De posse destas duas equacões, (3.51) e (3.52), as diferenças de energia livre para quaisquer dois subintervalos subsequentes, dentro do intervalo [0,1], representados por $U\left(\lambda_{i-1}\right)$ e $U\left(\lambda_{i+1}\right)$, podem ser obtidas a partir de uma única simulação, equilibrada para o estado intermediário representado por $U\left(\lambda_{i}\right)$. As duas médias resultantes desses cálculos para obter $\Delta G\left(\lambda_{i} \rightarrow \lambda_{i+1}\right)$ e $\Delta G\left(\lambda_{i} \rightarrow \lambda_{i-1}\right)$, por meio de uma única amostragem para ambas as direções, é conhecida na literatura como amostragem de passo duplo (double-wide sampling). Quando a amostragem é realizada numa única direção, dizemos que a amostragem é de passo único.

A variação total de energia livre é obtida somando-se as contribuições de cada intervalo na variável $\lambda_{i}$

$$
\Delta G_{\text {total }}=\sum_{i} \Delta G\left(\lambda_{i}\right)
$$


Como a energia livre é uma função de estado, $\Delta G_{\text {total }}$ não depende da escolha do parâmetro $\lambda$. Tem-se que a dependência funcional de $U(\mathbf{r})$ em $\lambda$ é obtida fazendose todos os termos do potencial intermolecular serem funções de $\lambda$. Desta forma, o potencial varia durante a perturbação do sistema entre os dois estados. Podemos também definir um novo parâmetro $\tau$ que varia de acordo com a seguinte equação:

$$
\tau\left(\lambda_{i}\right)=\lambda_{i} \tau_{F}+\left(1-\lambda_{i}\right) \tau_{I}
$$

em que $i, I$ e $F$ são os estados de referência, inicial e final, respectivamente. Portanto, o parâmetro $\tau$ pode ser usado, por exemplo, para causar a variação de cargas atômicas, dos parâmetros do potencial de Lennard-Jones, e também dos parâmetros geométricos de uma molécula, como ângulos e comprimentos de ligação e ângulos diedros. Assim, podemos obter a variação de energia livre nesses processos. 


\section{Capítulo 4}

\section{Resultados e Discussões}

Neste capítulo apresentaremos resultados dos estudos teóricos da molécula Óxido Mesitil (OM) isolada e em solução aquosa. Nesse estudo, analisamos os efeitos do solvente nas propriedades eletrônicas e conformacionais dessa molécula.

\subsection{Estudo da Molécula Isolada}

Nesta seção apresentaremos resultados para o estudo da molécula OM isolada. Apresentamos uma análise sobre a geometria do OM, em que foi feito um estudo referente ao nível de cálculo quântico utilizado. Isso se faz necessário porque não existem dados experimentais da sua estrutura. Desta forma, as estruturas das duas conformações, syn e anti, são obtidas através de cálculos quânticos, também é gerado um estado intermediário entre as duas conformações, estado de transição (TS), para que a barreira energética necessária para que ocorra a mudança syn-anti possa ser obtida. Em seguida, fazemos um estudo da diferença de energia livre entre os diferentes confórmeros do OM. Esse estudo nos dá a informação sobre a estabilidade e o valor da barreira energética. Analisamos também o seu espectro eletrônico de absorção com diferentes níveis de cálculos quânticos. Esse resultado será comparado a valores experimentais obtidos com solventes de baixa polaridade. Por fim, de posse desses 
estudos (geometria, energia livre e espectro eletrônico) apresentamos uma conclusão parcial à respeito da estabilidade da molécula, ou seja, qual confôrmero é mais estável em fase gasosa ou solvente de baixa polaridade.

\subsubsection{Análise Conformacional}

A molécula de óxido mesitil (OM) tem a fórmula molecular $\left(\left(\mathrm{CH}_{3}\right)_{2} \mathrm{C}=\mathrm{CHCOCH}_{3}\right)$ como mostrado no esquema da figura (4.1). Neste trabalho vamos estudar duas formas isoméricas do OM, syn e anti. O isomerismo é caracterizado pela existência de duas ou mais substâncias que apresentam fórmulas moleculares idênticas, mas diferem em suas formas estruturais. Neste caso, a diferença estrutural entre o isômero syn e o anti (ver figura (4.2) ) está na cadeia formada pelos átomos C1,C2,C3 e O4. Para o isomêro syn o ângulo diedral formado por essa cadeia de átomos possui o valor de $0^{\circ}$. Já o isômero anti possui o valor de $180^{\circ}$. Como não existem dados experimentais e nem teóricos da estrutura conformacional do OM, nós geramos a estrutura dessa molécula e a otimizamos a fim de obtermos a conformação mais estável. Para obtermos a barreira energética que deve ser vencida para que ocorra a transição de uma conformação à outra, nós geramos um estado intermediário, estado de transição $(T S)$. Esse estado tem a mesma fórmula molecular dos dois isômeros e sua estrutura é obtida fazendo uma otimização do estado de transição partindo do ângulo diedral formado pelos átomos $\mathrm{C} 1, \mathrm{C} 2, \mathrm{C} 3$ e $\mathrm{O} 4$ no valor de $90^{\circ}$. Para gerar as estruturas nós realizamos cálculos de mecânica quântica (QM) em dois níveis diferentes, usando Teoria do Funcional da Densidade com o funcional B3LYP e usando Teoria de Perturbação de Møller-Plesset de segunda ordem (MP2). Esses métodos incluem a correlação eletrônica de forma distinta. Adicionalmente usamos dois conjuntos de funções base: 6-31+G* e aug-ccpVDZ. Na figura (4.2) mostramos as geometrias otimizadas para essas 3 conformações. A seguir apresentamos uma análise quantitativa da sensibilidade da geometria quanto ao método/base utilizado. Na tabela (4.1) apresentamos resultados para o compri- 
mento das ligações com relação ao tipo de teoria utilizada (MP2 ou DFT-B3LYP).

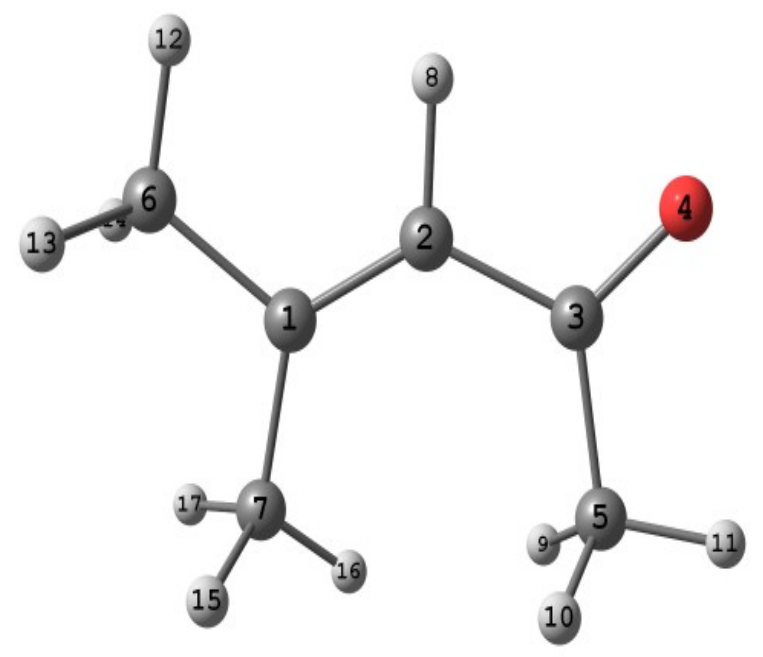

Figura 4.1: Ilustração da molécula de OM e numeração dos átomos.

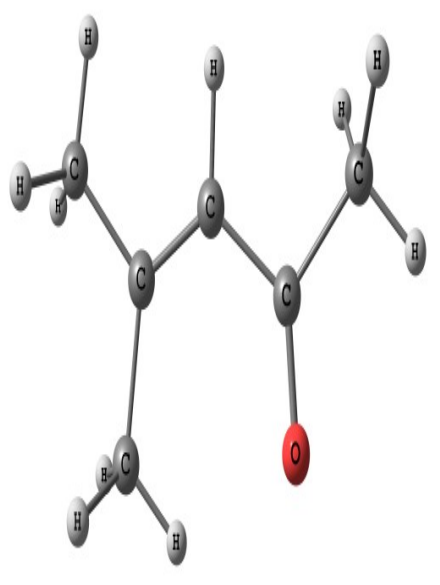

(a) syn

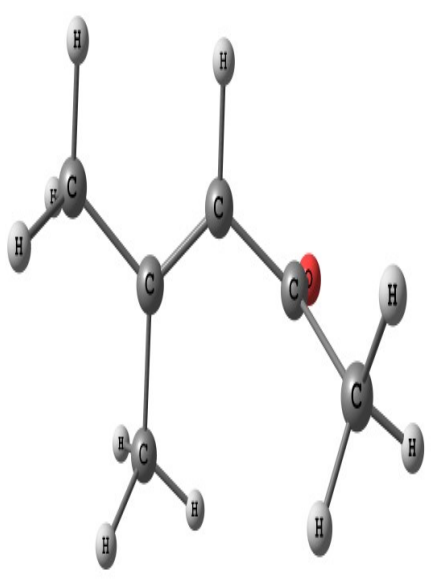

(b) TS

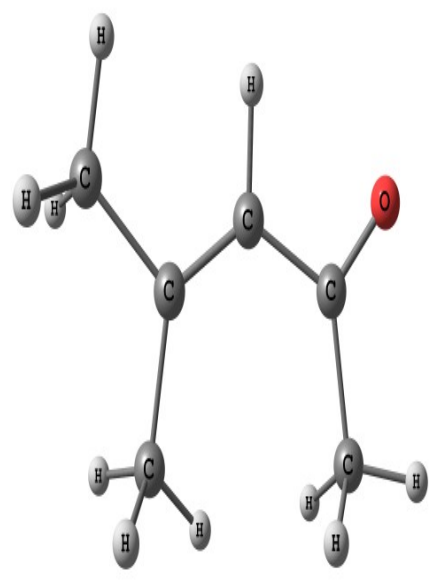

(c) anti

Figura 4.2: Ilustração dos isômeros do OM estudados, syn e anti, e o estado de transição, TS.

De um modo geral, observa-se que os métodos B3LYP e MP2 dão resultados muito semelhantes e que a escolha da função base também não parece influenciar muito o resultado quanto ao comprimento das ligações. Em média, as ligações apresentam um desvio de aproximadamente $0.003 \AA$ com relação as mudanças de métodos e funções 
Tabela 4.1: Distância das ligações entre os átomos obtidas nos diferentes níveis de cálculo e funções base.

\begin{tabular}{|c|c|c|c|c|}
\hline Dist. $(\AA)$ & B3LYP / & $6-31+\mathrm{G}^{*}$ & B3LYP/aug-cc-pVDZ & MP2/aug-cc-pVDZ \\
\hline & syn & anti & syn anti & syn anti \\
\hline $\mathrm{C} 1=\mathrm{C} 2$ & 1.354 & 1.354 & 1.3551 .354 & 1.3641 .364 \\
\hline $\mathrm{C} 1-\mathrm{C} 6$ & 1.509 & 1.512 & $1.507 \quad 1.510$ & 1.5101 .512 \\
\hline $\mathrm{C} 1-\mathrm{C} 7$ & 1.505 & 1.508 & 1.5031 .506 & 1.5071 .511 \\
\hline $\mathrm{C} 2-\mathrm{C} 3$ & 1.485 & 1.486 & 1.4851 .486 & 1.4911 .489 \\
\hline $\mathrm{C} 2-\mathrm{H} 8$ & 1.090 & 1.089 & $1.093 \quad 1.093$ & 1.0971 .097 \\
\hline $\mathrm{C} 3=\mathrm{O} 4$ & 1.228 & 1.229 & $1.226 \quad 1.227$ & 1.2371 .240 \\
\hline $\mathrm{C} 3-\mathrm{C} 5$ & 1.523 & 1.520 & 1.5201 .518 & 1.5201 .519 \\
\hline $\mathrm{C} 5-\mathrm{H} 9$ & 1.098 & 1.096 & 1.1011 .099 & 1.1031 .102 \\
\hline $\mathrm{C} 5-\mathrm{H} 10$ & 1.098 & 1.096 & 1.1011 .099 & 1.1031 .100 \\
\hline $\mathrm{C} 5-\mathrm{H} 11$ & 1.092 & 1.092 & $1.094 \quad 1.095$ & 1.0971 .098 \\
\hline $\mathrm{C} 6-\mathrm{H} 12$ & 1.094 & 1.094 & $1.097 \quad 1.096$ & 1.1001 .099 \\
\hline $\mathrm{C} 6-\mathrm{H} 13$ & 1.099 & 1.099 & 1.1021 .102 & 1.1041 .104 \\
\hline $\mathrm{C} 6-\mathrm{H} 14$ & 1.099 & 1.099 & 1.1021 .102 & 1.1041 .104 \\
\hline $\mathrm{C} 7-\mathrm{H} 15$ & 1.100 & 1.099 & $1.102 \quad 1.101$ & 1.1041 .104 \\
\hline $\mathrm{C} 7-\mathrm{H} 16$ & 1.089 & 1.086 & $1.091 \quad 1.090$ & 1.0941 .094 \\
\hline $\mathrm{C} 7-\mathrm{H} 17$ & 1.100 & 1.099 & 1.1021 .101 & 1.1041 .103 \\
\hline H8...O4 & 3.307 & 2.423 & 3.3112 .427 & $3.332 \quad 2.452$ \\
\hline $\mathrm{C} 2 \ldots \mathrm{O} 4$ & 2.405 & 2.324 & $2.404 \quad 2.324$ & $2.419 \quad 2.337$ \\
\hline
\end{tabular}


base. Um outro dado que se observa na tabela (4.1) é que o método MP2 apresenta um aumento no comprimento, na maioria das ligações, para os dois isômeros. As ligações mais sensíveis a mudança no nível de cálculo quântico e funções base são $\mathrm{C} 1=\mathrm{C} 2$ e $\mathrm{C} 3=\mathrm{O} 4$, ambas apresentam um desvio de 0.01 A quando mudamos o método quântico de B3LYP para MP2 e as funções base de 6-31+G* para aug-cc-pVDZ. A distância H8...O4 é cerca de $0.9 \AA$ maior no isômero syn, para todos os métodos/base. Para o estado de transição, também foi feita a mesma análise nessa conformação e os resultados são semelhantes aos apresentados tanto no que diz respeito a quais ligações são mais sensíveis ao nível de cálculo quântico como também nos valores dos desvios no comprimento das ligações.

Na tabela 4.2 apresentamos os resultados para os valores dos ângulos com relação a mudança no nível de cálculo. Novamente apresentamos somente os valores para os isômeros syn e anti.

Analisando esses ângulos quanto ao nível de cálculo quântico observa-se que tanto o método B3LYP quanto o MP2 apresentam resultados semelhantes, o mesmo acontece para as diferentes funções base. Um outro dado que se observa é que os ângulos em torno do carbono 3 (C3,C2,H8; C2,C3,O4 e C2,C3,C5) apresentam mudanças significativas, devido a troca syn $\rightarrow$ anti. O ângulo formado pelos átomos C3,C2,H8 apresenta um deslocamento de aproximadamente $4^{\circ}$ devido a mudança do isômero. Já os ângulos formado pelos átomos C2,C3,O4 e C2,C3,C5 mudam cerca de $7^{\circ}$ a $8^{\circ}$ do syn para o anti, respectivamente.

$\mathrm{Na}$ análise dos ângulos torcionais ou diedros, tabela (4.3), observa-se que não apresenta mudanças significativas devido ao nível de cálculo quântico. O que deve ser observado aqui são as diferenças angulares entre as conformações syn e anti. Nesse ponto, observa-se que o método MP2 com o conjunto de funções base aug-cc-pVDZ é o que apresenta, em alguns conjuntos de átomos, deslocamentos angulares significativos. Os diedros formados pelo $\mathrm{CH}_{3}$ dos carbonos 7 e 5 (todos os diedros envolvendo os 
hidrogênios 15,16 e 17 e 9,10 e 11) indicam uma rotação desses grupos de cerca de $17^{\circ}$ e $10^{\circ}$, respectivamente, do syn para o anti. No nível MP2 também pode-se observar uma quebra na planaridade da cadeia $\mathrm{C} 1, \mathrm{C} 2, \mathrm{C} 3, \mathrm{C} 5$ e $\mathrm{C} 1, \mathrm{C} 2, \mathrm{C} 3, \mathrm{O} 4 \mathrm{em}$ cerca de $7^{\circ}$ no isômero anti.

Tabela 4.2: Valores dos ângulos de ligação para as conformações obtidos nos diferentes níveis de cálculos QM.

\begin{tabular}{|c|c|c|c|c|c|c|}
\hline \multirow[t]{2}{*}{$\operatorname{Ang}\left({ }^{\circ}\right)$. } & \multicolumn{2}{|c|}{ B3LYP $/ 6-31+\mathrm{G}^{*}$} & \multicolumn{2}{|c|}{ B3LYP/aug-cc-pVDZ } & \multicolumn{2}{|c|}{ MP2/aug-cc-pVDZ } \\
\hline & syn & anti & syn & anti & syn & anti \\
\hline $\mathrm{C} 2, \mathrm{C} 1, \mathrm{C} 6$ & 119.9 & 118.9 & 119.9 & 118.9 & 119.8 & 119.0 \\
\hline $\mathrm{C} 2, \mathrm{C} 1, \mathrm{C} 7$ & 125.2 & 128.0 & 125.2 & 127.8 & 125.3 & 127.5 \\
\hline $\mathrm{C} 6, \mathrm{C} 1, \mathrm{C} 7$ & 114.9 & 113.1 & 114.9 & 113.3 & 114.9 & 113.5 \\
\hline $\mathrm{C} 1, \mathrm{C} 2, \mathrm{C} 3$ & 128.3 & 133.1 & 128.1 & 132.9 & 127.9 & 131.5 \\
\hline $\mathrm{C} 1, \mathrm{C} 2, \mathrm{H} 8$ & 117.4 & 117.1 & 117.4 & 117.1 & 117.3 & 117.4 \\
\hline $\mathrm{C} 3, \mathrm{C} 2, \mathrm{H} 8$ & 114.3 & 109.8 & 114.5 & 110.0 & 114.8 & 111.2 \\
\hline $\mathrm{C} 2, \mathrm{C} 3, \mathrm{O} 4$ & 124.7 & 117.5 & 124.7 & 117.5 & 124.7 & 117.8 \\
\hline $\mathrm{C} 2, \mathrm{C} 3, \mathrm{C} 5$ & 115.0 & 123.6 & 115.0 & 123.4 & 114.6 & 122.8 \\
\hline $\mathrm{O} 4, \mathrm{C} 3, \mathrm{C} 5$ & 120.3 & 119.0 & 120.3 & 119.0 & 120.8 & 119.4 \\
\hline $\mathrm{C} 3, \mathrm{C} 5, \mathrm{H} 9$ & 110.5 & 111.3 & 110.2 & 111.1 & 109.9 & 110.2 \\
\hline C3,C5,H10 & 110.5 & 111.3 & 110.2 & 111.1 & 109.9 & 111.5 \\
\hline $\mathrm{C} 3, \mathrm{C} 5, \mathrm{H} 11$ & 109.8 & 108.2 & 109.8 & 108.1 & 109.7 & 107.9 \\
\hline H9,C5,H10 & 107.0 & 107.9 & 106.9 & 107.9 & 107.3 & 108.2 \\
\hline H9,C5,H11 & 109.5 & 109.0 & 109.8 & 109.3 & 110.0 & 108.8 \\
\hline $\mathrm{H} 10, \mathrm{C} 5, \mathrm{H} 11$ & 109.5 & 109.0 & 109.8 & 109.3 & 110.0 & 110.1 \\
\hline
\end{tabular}




$\begin{array}{crrrrrr}\text { C1,C6,H12 } & 112.5 & 112.2 & 112.4 & 112.1 & 112.2 & 112.0 \\ \text { C1,C6,H13 } & 110.5 & 110.6 & 110.3 & 110.5 & 110.1 & 110.1 \\ \text { C1,C6,H14 } & 110.5 & 110.6 & 110.3 & 110.5 & 110.1 & 110.2 \\ \text { H12,C6,H13 } & 108.4 & 108.3 & 108.6 & 108.6 & 108.8 & 108.7 \\ \text { H12,C6,H14 } & 108.4 & 108.3 & 108.6 & 108.6 & 108.8 & 108.8 \\ \text { H13,C6,H14 } & 106.4 & 106.5 & 106.4 & 106.4 & 106.8 & 106.9 \\ \text { C1,C7,H15 } & 109.7 & 109.7 & 109.5 & 109.6 & 109.2 & 109.7 \\ \text { C1,C7,H16 } & 112.4 & 114.7 & 112.3 & 114.6 & 112.5 & 114.2 \\ \text { C1,C7,H17 } & 109.7 & 109.7 & 109.5 & 109.6 & 109.2 & 109.2 \\ \text { H15,C7,H16 } & 109.3 & 108.0 & 109.6 & 108.3 & 109.6 & 108.5 \\ \text { H15,C7,H17 } & 106.2 & 106.2 & 106.1 & 106.1 & 106.6 & 106.7 \\ \text { H16,C7,H17 } & 108.0 & 108.0 & 110.0 & 108.3 & 109.6 & 108.2\end{array}$

Tabela 4.3: Valores diedrais para as conformações e os respectivos métodos utilizados.

\begin{tabular}{|c|c|c|c|c|c|c|}
\hline \multirow[t]{2}{*}{ Diedros $\left(^{\circ}\right)$} & \multicolumn{2}{|c|}{ B3LYP $/ 6-31+\mathrm{G}^{*}$} & \multicolumn{2}{|c|}{ B3LYP/aug-cc-pVDZ } & \multicolumn{2}{|c|}{ MP2/aug-cc-pVDZ } \\
\hline & syn & anti & syn & anti & syn & anti \\
\hline $\mathrm{C} 6, \mathrm{C} 1, \mathrm{C} 2, \mathrm{C} 3$ & 180.0 & 180.0 & 180.0 & 180.0 & 180.0 & 178.6 \\
\hline $\mathrm{C} 6, \mathrm{C} 1, \mathrm{C} 2, \mathrm{H} 8$ & 0.0 & 0.0 & 0.0 & 0.0 & 0.0 & -1.7 \\
\hline $\mathrm{C} 7, \mathrm{C} 1, \mathrm{C} 2, \mathrm{C} 3$ & 0.0 & 0.0 & 0.0 & 0.0 & 0.0 & -1.0 \\
\hline $\mathrm{C} 7, \mathrm{C} 1, \mathrm{C} 2, \mathrm{H} 8$ & 180.0 & 180.0 & 180.0 & 180.0 & 180.0 & 178.8 \\
\hline $\mathrm{C} 2, \mathrm{C} 1, \mathrm{C} 6, \mathrm{H} 12$ & 0.0 & -0.1 & 0.0 & -0.1 & 0.0 & -3.1 \\
\hline
\end{tabular}




\begin{tabular}{|c|c|c|c|}
\hline $\mathrm{C} 2, \mathrm{C} 1, \mathrm{C} 6, \mathrm{H} 13$ & $-121.3-121.2$ & $-121.4-121.3$ & $-121.2 \quad-124.2$ \\
\hline $\mathrm{C} 2, \mathrm{C} 1, \mathrm{C} 6, \mathrm{H} 14$ & $121.3 \quad 121.1$ & 121.4121 .2 & 121.2118 .2 \\
\hline $\mathrm{C} 7, \mathrm{C} 1, \mathrm{C} 6, \mathrm{H} 12$ & 180.0179 .9 & $\begin{array}{ll}180.0 & 179.9\end{array}$ & $\begin{array}{ll}180.0 & 176.5\end{array}$ \\
\hline $\mathrm{C} 7, \mathrm{C} 1, \mathrm{C} 6, \mathrm{H} 13$ & $58.7 \quad 58.8$ & $58.6 \quad 58.7$ & $58.8 \quad 55.4$ \\
\hline $\mathrm{C} 7, \mathrm{C} 1, \mathrm{C} 6, \mathrm{H} 14$ & $\begin{array}{lll}-58.7 & -58.9\end{array}$ & $\begin{array}{lll}-58.6 & -58.8\end{array}$ & $-58.8-62.3$ \\
\hline $\mathrm{C} 2, \mathrm{C} 1, \mathrm{C} 7, \mathrm{H} 15$ & 121.9121 .6 & 122.0121 .8 & $121.9 \quad 105.1$ \\
\hline $\mathrm{C} 2, \mathrm{C} 1, \mathrm{C} 7, \mathrm{H} 16$ & $0.0-0.2$ & $0.0-0.2$ & $0.0-17.0$ \\
\hline $\mathrm{C} 2, \mathrm{C} 1, \mathrm{C} 7, \mathrm{H} 17$ & $-121.8-122.0$ & $-122.0-122.1$ & $-121.9-138.2$ \\
\hline $\mathrm{C} 6, \mathrm{C} 1, \mathrm{C} 7, \mathrm{H} 15$ & $-58.2 \quad-58.4$ & $-58.0-58.2$ & $-58.1 \quad-74.4$ \\
\hline $\mathrm{C} 6, \mathrm{C} 1, \mathrm{C} 7, \mathrm{H} 16$ & $180.0 \quad 179.8$ & 180.0179 .8 & $180.0 \quad 163.5$ \\
\hline $\mathrm{C} 6, \mathrm{C} 1, \mathrm{C} 7, \mathrm{H} 17$ & $58.2 \quad 58.0$ & $58.0 \quad 57.9$ & $58.1 \quad 42.2$ \\
\hline $\mathrm{C} 1, \mathrm{C} 2, \mathrm{C} 3, \mathrm{O} 4$ & $0.0 \quad 180.0$ & $0.0 \quad 180.0$ & $0.0 \quad 173.5$ \\
\hline $\mathrm{C} 1, \mathrm{C} 2, \mathrm{C} 3, \mathrm{C} 5$ & $180.0-0.1$ & $180.0 \quad 0.0$ & $180.0-7.8$ \\
\hline $\mathrm{H} 8, \mathrm{C} 2, \mathrm{C} 3, \mathrm{O} 4$ & $180.0-0.0$ & $180.0 \quad 0.0$ & $180.0 \quad 6.2$ \\
\hline $\mathrm{H} 8, \mathrm{C} 2, \mathrm{C} 3, \mathrm{C} 5$ & 0.0180 .0 & $0.0 \quad 180.0$ & $0.0 \quad 172.4$ \\
\hline $\mathrm{C} 2, \mathrm{C} 3, \mathrm{C} 5, \mathrm{H} 9$ & $-59.1-60.1$ & $-58.9-59.9$ & $-58.9-47.5$ \\
\hline C2,C3,C5,H10 & $59.1 \quad 60.3$ & $58.9 \quad 60.1$ & $58.9 \quad 72.7$ \\
\hline C2,C3,C5,H11 & $180.0 \quad 179.9$ & $180.0 \quad 179.9$ & $180.0 \quad 168.6$ \\
\hline $\mathrm{O} 4, \mathrm{C} 3, \mathrm{C} 5, \mathrm{H} 9$ & $121.0 \quad 119.9$ & $121.1 \quad 120.0$ & $121.1 \quad 131.2$ \\
\hline $\mathrm{O} 4, \mathrm{C} 3, \mathrm{C} 5, \mathrm{H} 10$ & $-120.9-119.7$ & $-121.1-119.9$ & $-121.1-108.6$ \\
\hline O4,C3,C5,H11 & $0.0 \quad 0.0$ & $0.0 \quad 0.1$ & $0.0 \quad 10.1$ \\
\hline
\end{tabular}


Uma vez obtida as geometrias otimizadas para cada conformação, nós calculamos as variações de energia na mudança da conformação syn para o TS e para o isômero anti, a fim de verificarmos a sensibilidade quanto ao nível de cálculo utilizado.

Tabela 4.4: Tabela com as variações da energia (kcal/mol) calculada com diferentes níveis de cálculos QM.

\begin{tabular}{lcc}
\hline \hline Método/base & $\Delta E($ syn $\rightarrow$ TS $)$ & $\Delta E($ syn $\rightarrow$ anti $)$ \\
\hline B3LYP /6-31+G* & 6.03 & 2.98 \\
B3LYP/aug-cc-pVDZ & 5.61 & 2.75 \\
MP2/aug-cc-pVDZ & 4.40 & 1.95 \\
\hline \hline
\end{tabular}

Comparando os resultados B3LYP com diferentes bases (tabela (4.4) observa-se que, embora as geometrias não sejam sensíveis a essa mudança de base, as diferenças de energia ainda não estão saturadas com a base $6-31+\mathrm{G}^{*}$. Isso mostra a necessidade de utilização de uma base maior para o cálculo da energia relativa dos isômeros. Por outro lado, usando a base maior (aug-cc-pVDZ) e mudando o método B3LYP para MP2 também observa-se diferenças nas energias relativas. As energias relativas obtidas pelo método MP2 são menores do que as obtidas usando o funcional de densidade B3LYP, apresentando uma redução cerca de $1.2 \mathrm{kcal} / \mathrm{mol}$ na barreira e $0.8 \mathrm{kcal} / \mathrm{mol}$ na diferença devido a mudança conformacional syn $\rightarrow$ anti.

As correções na energia dos isômeros devido a energia de ponto zero, $E_{Z P E}$, e devido a energia térmica, $E_{T}$, foram obtidas calculando as frequências vibracionais nos níveis B3LYP e MP2 e as funções base foram as mesmas utilizadas na otimização da energia. Na tabela (4.5) apresentamos esses valores onde o método/base indica o nível de cálculo quântico para a otimização da energia e cálculo das frequências vibracionais.

Nesses cálculos também foram obtidas as variações de energia livre. Os resultados são apresentados na tabela 4.6. 
Tabela 4.5: Correções térmicas e energia de ponto zero relativas $(\mathrm{kcal} / \mathrm{mol})$ obtidas com diferentes níves de cálculos QM.

\begin{tabular}{lcccc}
\hline \hline Método/base & $\Delta E_{Z P E}$ & \multicolumn{3}{c}{$\Delta E_{T}$} \\
\hline & syn $\rightarrow T S$ & syn $\rightarrow$ anti & syn $\rightarrow T S$ & syn $\rightarrow$ anti \\
B3LYP $/ 6-31+\mathrm{G}^{*}$ & -0.33 & 0.45 & -0.37 & 0.86 \\
$\mathrm{~B} 3 \mathrm{LYP} /$ aug-cc-pVDZ & -0.37 & 0.37 & -0.91 & 0.24 \\
$\mathrm{MP} 2 /$ aug-cc-pVDZ & -0.37 & 0.35 & -0.35 & 0.76 \\
\hline \hline
\end{tabular}

Tabela 4.6: Tabela com as variações da energia livre ( $\mathrm{kcal} / \mathrm{mol}$ ) calculada com diferentes níveis de cálculos QM.

\begin{tabular}{lcc}
\hline \hline Método/base & $\Delta G($ syn $\rightarrow T S)$ & $\Delta G($ syn $\rightarrow$ anti $)$ \\
\hline B3LYP/6-31+G* & 5.93 & 2.20 \\
B3LYP/aug-cc-pVDZ & 6.80 & 2.00 \\
MP2/aug-cc-pVDZ & 3.57 & 1.34 \\
MP2/aug-cc-pVDZ//B3LYP/6-31+G* & 4.10 & 2.42 \\
\hline \hline
\end{tabular}


Na tabela 4.6 o nível de cálculo quântico MP2/aug-cc-pVDZ//B3LYP/6-31+G* significa que a geometria e a energia foram otimizadas com o método B3LYP com as funções base 6-31+ $\mathrm{G}^{*}$, as correções térmicas e energia de ponto zero forma obtidas com MP2/aug-cc-pVDZ. Observa-se na tabela (4.6) que usando o método MP2 a barreira, $\Delta G(\operatorname{syn} \rightarrow T S)$, diminui cerca de $3.0 \mathrm{kcal} / \mathrm{mol}$ comparativamente ao valor obtido com B3LYP. Um dado que se verifica nos diferentes métodos é que o isômero syn é a conformação mais estável e que o método MP2 é o que dá menor energia relativa.

Uma vez realizado o cálculo da variação da energia livre associada a mudança syn $\rightarrow$ anti em fase gasosa. Nós podemos obter as populações correspondentes a cada conformação em fase gasosa, o que deve ser compatível com solventes apolares. Experimental, a variação de energia livre associada a mudança syn $\rightarrow$ anti pode ser obtida pela constante de equilíbrio $K$ através da seguinte relação

$$
\Delta G=-R T \ln K
$$

onde $R$ é a constante dos gases e $T$ a temperatura. Aqui, através de cálculo teórico nós determinamos a energia livre. Portanto, nós podemos determinar a constante de equilíbrio $K$

$$
K=e^{-\Delta G / R T}
$$

que também é dado pela razão entre a concentração do produto e a concentração dos reagentes, que no nosso caso será

$$
K=\frac{[a n t i]}{[s y n]}
$$

onde $[$ anti $]+[$ syn $]=1$. Combinando as equações 4.2 e $(4.3)$ nós podemos determinar as concentrações (ou populações) para cada conformação. Na tabela 4.7) apresentamos a população do isômero syn em fase gasosa juntamente com a energia livre associada à mudança conformacional syn $\rightarrow$ anti. 
Tabela 4.7: Tabela com as variações da energia livre $(\mathrm{kcal} / \mathrm{mol})$ calculada com diferentes níveis de cálculos QM.

\begin{tabular}{lcc}
\hline \hline Método/base & $\Delta G($ syn $\rightarrow$ anti $)$ & População (\%) syn \\
\hline B3LYP/6-31+G* & 2.20 & 98 \\
B3LYP/aug-cc-pVDZ & 2.00 & 97 \\
MP2/aug-cc-pVDZ & 1.34 & 91 \\
MP2/aug-cc-pVDZ//B3LYP/6-31+G* & 2.42 & 98 \\
\hline \hline
\end{tabular}

Nós também cálculamos o momento de dipolo de cada conformação isolada. Para comparação teremos o valores experimentais de $2.70 \mathrm{D}$ [1] e 2.80D [9] para o óxido mesitil obtido em solução de baixa polaridade e uma estimativa de $\sim 3.7 \mathrm{D}$ para o isômero anti $[9]$. Na tabela (4.8) apresentamos os valores teóricos para o momento de dipolo do OM nas três conformações, obtidos usando diferentes métodos e funções base, e o valor experimental.

Tabela 4.8: Dipolo(D) do OM nas conformações syn, TS e anti em fase gasosa calculado usando diferentes níveis de cálculos QM.

\begin{tabular}{lccc}
\hline \hline Método/base & \multicolumn{3}{c}{$\mu(D)$} \\
\hline & syn & TS & anti \\
B3LYP/6-31+G* & 3.14 & 3.30 & 4.38 \\
B3LYP/aug-cc-pVDZ & 3.00 & 3.22 & 4.29 \\
MP2/aug-cc-pVDZ & 2.80 & 3.00 & 3.97 \\
MP2/aug-cc-pVDZ//B3LYP/6-31+ $\mathrm{G}^{*}$ & 2.77 & 3.01 & 3.90 \\
Experimental |9 & 2.80 & & $\sim 3.7$ \\
\hline \hline
\end{tabular}


Observa-se na tabela 4.8 que o método MP2 apresenta, para as três conformações, um momento de dipolo menor comparado ao obtido com B3LYP e que os dipolos obtidos em ambas geometrias (MP2 e B3LYP) são muito semelhantes. Observa-se também que os isômeros syn e anti apresentam um momento de dipolo em boa concordância com os valores experimentais para o dipolo do OM, em particular $\mu=2.77 \mathrm{D}$ e $\mu=3.90 \mathrm{D}$ obtidos com MP2/aug-cc-pVDZ//B3LYP/6-31+G, respectivamente. Portanto, indica que o isômero syn seria a conformação predominante em solventes de baixa polaridade, em concordância com o $\Delta G($ syn $\rightarrow$ anti $)$ calculado, que mostra que a conformação syn teria uma população de $98 \%$ em solventes de baixa polaridade. .

\subsubsection{Espectro de Absorção}

O espectro eletrônico de absorção do OM foi obtido experimentalmente por Kosower [9] para diferentes solventes, variando a polaridade desde o iso-octano (baixa polaridade) até a água (alta polaridade). Nesse experimento foi observado a existência de uma banda menos intensa caracterizada por uma transição $n-\pi^{*}$ e uma banda mais intensa caracterizada por uma transição $\pi-\pi^{*}$. Neste trabalho, vamos estudar apenas a transição mais intensa, uma vez que a análise do efeito do solvente será feita apenas na $2^{\mathrm{a}}$ banda, pois os resultados da transição $n-\pi^{*}$ estão afetados pela presença do isômero óxido isomesitil.

Com o intuito de descrever o espectro eletrônico de absorção da molécula OM nós realizamos cálculos quânticos usando Teoria do Funcional da Densidade Dependente do Tempo (TD-DFT), nessa etapa as geometrias para os isômeros syn e anti foram obtidas com o método B3LYP e as funções base 6-31+G*. Para a obtenção da energia eletrônica de absorção nós realizamos cálculos QM com diferentes níveis e bases, como comparação teremos o resultado experimental para a energia de absorção $\left(\mathrm{cm}^{-1}\right)$ do OM em iso-octano, $43365 \mathrm{~cm}^{-1}$, para a transição mais intensa $\left(\pi-\pi^{*}\right)$, obtida a partir da tabela (1.1). O iso-octano foi escolhido para comparação com os nossos cálculos 
realizados com a molécula isolada por ser um solvente de mais baixa polaridade.

No cálculo teórico a transição mais intensa ocorre do orbital mais alto ocupado, HOMO (Highest Occupied Molecular Orbital), para o mais baixo desocupado, LUMO (Lowest Unoccupied Molecular Orbital). Esse orbitais são ilustrados na figura (4.3).

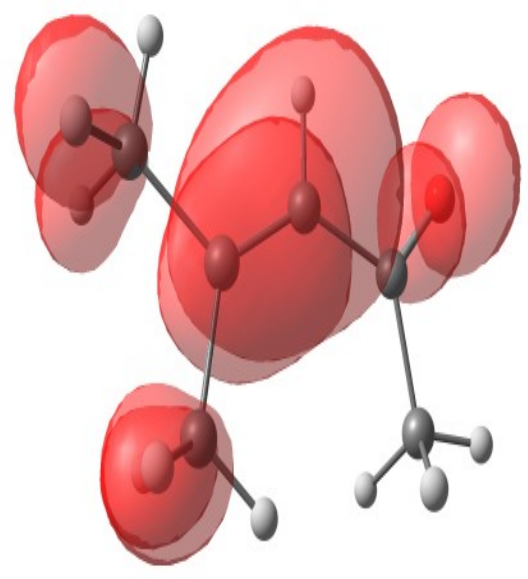

(a) HOMO

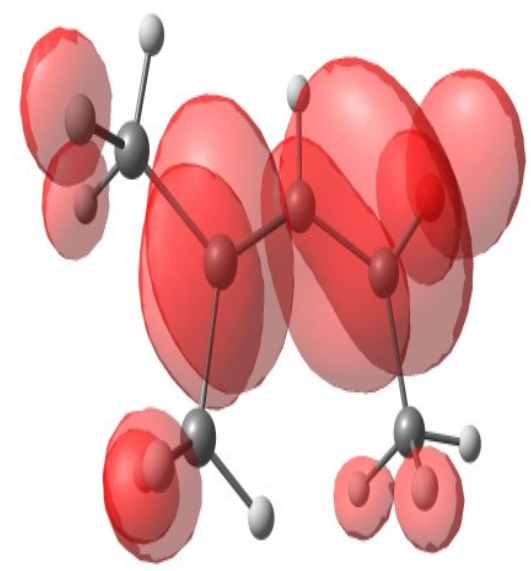

(b) LUMO

Figura 4.3: Ilustração dos orbitais envolvidos na transição mais intensa $\left(\pi-\pi^{*}\right)$ do $\mathrm{OM}$ isolado.

Observa-se na figura (4.3) que o orbital HOMO é perpendicular ao plano da molécula caracterizando um orbital $\pi$. O orbital LUMO também é perpendicular ao plano da molécula e esse orbital é caracterizado como sendo um orbital $\pi^{*}$. A transição eletrônica ocorre dos átomos C1,C2 para os átomos C2,C3.

Na tabela 4.9 apresentamos os resultados obtidos usando o funcional B3LYP e diversas funções base.

Observa-se na tabela (4.9) que a mudança da base $6-311++\mathrm{G}^{*}$ para $6-311++\mathrm{G}^{* *}$ provoca uma diminuição de apenas $27 \mathrm{~cm}^{-1}$ na energia de absorção, indicando que as bases de Pople já estão saturadas. Por outro lado a mudança de uma função double $-\zeta$ para uma triple - $\zeta$ nas bases de Dunning (aug-cc-pVDZ e aug-cc-pVTZ, respectivamente) provoca um deslocamento na energia de $33 \mathrm{~cm}^{-1}$ e $10 \mathrm{~cm}^{-1}$ para os isômeros 
Tabela 4.9: Energia de absorção $\left(\mathrm{cm}^{-1}\right)$ da transição $\pi-\pi^{*}$ do $\mathrm{OM}$ em função da base e o respectivo tempo de $\mathrm{CPU}$ envolvido no cálculo.

\begin{tabular}{|c|c|c|c|c|}
\hline Método/base & syn & anti & $\mathrm{CPU}$ & Exp. em iso-octano \\
\hline B3LYP /6-31+G* & 44084 & 45273 & 0h-16-mi & \\
\hline B3LYP $/ 6-31++\mathrm{G}^{*}$ & 43942 & 45053 & 0h-19-mi & \\
\hline B3LYP $/ 6-311+\mathrm{G}^{*}$ & 43810 & 45053 & 0h-24-mi & \\
\hline B3LYP $/ 6-311+\mathrm{G}^{* *}$ & 43783 & 45031 & 0h-35-mi & $43365^{a}$ \\
\hline B3LYP $/ 6-311++\mathrm{G}^{*}$ & 43712 & 44919 & 0h-31-mi & \\
\hline B3LYP $/ 6-311++\mathrm{G}^{* *}$ & 43685 & 44895 & 0h-43-mi & \\
\hline B3LYP/cc-pVDZ & 44998 & 46290 & 1h-19-mi & \\
\hline B3LYP/aug-cc-pVDZ & 43543 & 44735 & 2h-26-mi & \\
\hline B3LYP/aug-cc-pVTZ & 43510 & 44725 & 16h-14-mi & \\
\hline
\end{tabular}


syn e anti, respectivamente. Onde novamente se verifica a saturação da função base. Analisando a tabela 4.9 observa-se que o nosso melhor resultado, comparativamente com o experimental, para o espectro de absorção do OM em fase gasosa é de 43510 $\mathrm{cm}^{-1}$ obtido com a base aug-cc-pVTZ, porém o tempo computacional é muito grande, para este conjunto de funções base teremos um deslocamento de $1215 \mathrm{~cm}^{-1}$ devido a mudança conformacional syn-anti. Já a base $6-311++\mathrm{G}^{* *}$ apresenta uma energia de $43685 \mathrm{~cm}^{-1}$, verifica-se que também consegue descrever de forma satisfatória a transição eletrônica com a vantagem de ser menos custosa computacionalmente. O custo computacional será importante mais adiante quando descrevemos o efeito do solvente no espectro de absorção do OM. Nessa base o deslocamento devido a mudança syn $\rightarrow$ anti é de $1210 \mathrm{~cm}^{-1}$, em boa concordância com a melhor base.

Para testar a dependência desses nossos resultados com o tipo de funcional escolhido, também realizamos cálculos os funcionais PBE e BHandHLYP. Os resultados são apresentados nas tabelas 4.10 e 4.11, respectivamente.

Observa-se que o funcional PBE apresenta valores bem inferiores comparado com o valor experimental, o comportamento das funções base é semelhante ao descrito para o funcional B3LYP. O deslocamento na energia devido a mudança conformacional usando o conjunto de funções base aug-cc-pVTZ é de $887 \mathrm{~cm}^{-1}$.

Observa-se da tabela (4.11) que o funcional BHandHLYP apresenta valores bem superiores ao valor experimental, as funções base apresentam um comportamento de convergência semelhante ao descrito para o funcional B3LYP analisado anteriormente. Usando o conjunto de funções base aug-cc-pVTZ nós obtemos um deslocamento na energia de $1724 \mathrm{~cm}^{-1}$ devido a mudança conformacional. O estudo do espectro de absorção com diferentes funcionais nos mostra a importância em se ter um funcional que descreva bem a propriedade em que estamos interessados.

De posse dos resultados para o estudo da molécula isolada: momento de dipolo, energia livre e espectro de absorção, nós temos que em fase gasosa a conformação mais 
Tabela 4.10: Energia de absorção $\left(\mathrm{cm}^{-1}\right)$ da transição $\pi-\pi^{*}$ do OM em função da base. Funcional PBE.

\begin{tabular}{lrrr}
\hline \hline Método/base & syn & anti & Exp. em iso-octano \\
\hline $\mathrm{PBE} / 6-31+\mathrm{G}^{*}$ & 42013 & 42911 & \\
$\mathrm{PBE} / 6-31++\mathrm{G}^{*}$ & 41302 & 42194 & $43365^{a}$ \\
$\mathrm{PBE} / 6-311+\mathrm{G}^{*}$ & 41792 & 42731 & \\
$\mathrm{PBE} / 6-311+\mathrm{G}^{* *}$ & 41766 & 42699 & \\
$\mathrm{PBE} / 6-311++\mathrm{G}^{*}$ & 41346 & 42258 & \\
$\mathrm{PBE} / 6-311++\mathrm{G}^{* *}$ & 41300 & 42205 & \\
$\mathrm{PBE} / \mathrm{cc}-\mathrm{pVDZ}$ & 42869 & 43856 & \\
$\mathrm{PBE} /$ aug-cc-pVDZ & 40943 & 41841 & \\
$\mathrm{PBE} /$ aug-cc-pVTZ & 40975 & 41862 & \\
\hline \hline a ref. |9| & &
\end{tabular}


Tabela 4.11: Energia de absorção $\left(\mathrm{cm}^{-1}\right)$ da transição $\pi-\pi^{*}$ do $\mathrm{OM}$ em função da base. Funcional BHandHLYP.

\begin{tabular}{lrrr}
\hline \hline Método/base & syn & anti & Exp. em iso-octano \\
\hline BHandHLYP $/ 6-31+\mathrm{G}^{*}$ & 46753 & 48407 & \\
BHandHLYP $/ 6-31++\mathrm{G}^{*}$ & 46661 & 48323 & \\
BHandHLYP $/ 6-311+\mathrm{G}^{*}$ & 46434 & 48130 & $43365^{a}$ \\
BHandHLYP $/ 6-311+\mathrm{G}^{* *}$ & 46414 & 48114 & \\
BHandHLYP $/ 6-311++\mathrm{G}^{*}$ & 46369 & 48082 & \\
BHandHLYP/6-311++G** & 46352 & 48065 & \\
BHandHLYP/cc-pVDZ & 47651 & 49307 & \\
BHandHLYP/aug-cc-pVDZ & 46194 & 47920 \\
BHandHLYP/aug-cc-pVTZ & 46134 & 47858 & \\
\hline \hline
\end{tabular}

${ }^{a}$ ref. $|9|$ 
estável da molécula de OM é o isômero syn. Observamos também que a geometria do OM não é muito sensível à mudança no nível de cálculo QM. Verificamos que o método MP2 é o que apresenta menores valores para a diferença de energia livre entre os isômeros syn-anti. Quanto a análise do espectro o funcional B3LYP foi o que melhor descreveu a transição mais intensa $\left(\pi-\pi^{*}\right)$ do OM em fase gasosa (ou solvente de baixa polaridade), observou-se também a sensibilidade dessa propriedade com relação à função base utilizada e os funcionais.

\subsection{Estudo da Molécula em Solução Aquosa}

Nessa seção apresentaremos os resultados obtidos para o estudo da molécula de OM solvatada em água. Novamente levamos em consideração o estudo das conformações syn, TS e anti. As estruturas do OM solvatado em água foram geradas através de simulações computacionais de líquidos moleculares, com o método Monte Carlo Metropolis. Geradas as configurações, analisamos a função de auto-correlação da energia para definir o intervalo de correlação e selecionar apenas as configurações estatisticamente descorrelacionadas. Essas configurações foram submetidas aos cálculos quânticos para a determinação do espectro eletrônico de absorção da molécula em solução. Para realizarmos esses cálculos foi necessário definir qual região do sistema solvatado seria tratada quanticamente. Como o número de moléculas de água utilizadas na simulação é muito grande, para realizar os cálculos quânticos nós reduzimos esse número análisando as RDF's (Função de Distribuição Radial) que nos dá informação à respeito das camadas de solvatação, ou seja, quais moléculas de água estão mais próximas do soluto e portanto terão interações soluto-solvente mais importantes. Um fator decorrente dessa separação entre as simulações clássicas e os cálculos quânticos é a não inclusão da polarização do soluto na presença do solvente. Esse efeito é incluido neste trabalho através de um processo iterativo em que as cargas do soluto são ajustadas na presença do solvente. Uma vez realizado todos os procedimentos: geração das 
configurações do soluto solvatado, obtenção das configurações descorrelacionadas, distribuição do solvente em torno do soluto, processo de polarização do soluto na presença do solvente, nós cálculamos a energia livre de solvatação usando Teoria de Perturbação Termodinâmica (TPT) para verificarmos qual confôrmero é mais estável em água e finalmente apresentamos o efeito do solvente no espectro eletrônico da molécula através do estudo do deslocamento solvatocrômico usando Teoria do Funcional da Densidade Dependente do Tempo (TD-DFT).

\subsubsection{Detalhes da Simulação}

Para simularmos a molécula de OM em água usamos o método Monte Carlo que está implementado no programa DICE. O nosso estudo foi feito considerando as moléculas rígidas, onde a geometria do OM foi obtida realizando cálculos quânticos no nível B3LYP/6-31+G*. O modelo usado para descrever a água foi o SPC [101].

O ensemble utilizado foi o NPT, em que são mantidos fixos o número de partículas, a pressão e a temperatura. No nosso caso o número de partículas foi de 1 óxido mesitil e 500 águas, a pressão de 1 atm e a temperatura de 298K. As simulações foram realizadas utilizando condições periódicas de contorno numa caixa cúbica de lado aproximadamente igual a $24.9 \AA$.

Para reproduzir a interação intermolecular entre o soluto e o solvente usamos o potencial de Lennard-Jones adicionado à um termo Coulombiano, equação (3.31). Como a molécula de $\mathrm{OM}$ não está parametrizada nós obtivemos os parâmetros $\epsilon$ e $\sigma$ para o potencial de Lennard-Jones por semelhança com grupos funcionais que possuem parametrização. Isso foi feito usando o campo de força OPLS [102]. Para as cargas atômicas do termo de Coulomb foi feito uma análise para verificar se as cargas do OPLS reproduzem bem o comportamento eletrostático da molécula. Essa análise foi feita comparando as cargas obtidas através do campo de força com as cargas obtidas em fase gasosa realizando cálculos quânticos no nível MP2/aug-cc-pVDZ utilizando o 
ajuste do potencial eletrostático com o procedimento CHELPG. Esses conjuntos de cargas são apresentados na tabela (4.12) para os isômeros syn e anti.

Na tabela 4.12, a numeração dos átomos é a mesma da figura 4.1). Observa-se nessa tabela que o dipolo obtido com as cargas do campo de força OPLS apresenta uma mudança quando comparado ao dipolo obtido através do cálculo quântico em fase gasosa. O isômero syn é o que apresenta um maior dipolo com as cargas OPLS. O que não é razoável pois o campo de força deve seguir uma descrição semelhante do potencial eletrostático obtido quânticamente. Com relação às cargas de cada átomo a maior diferença entre os dois conjutos, OPLS e cálculo quântico, está nos átomos C1 e C2. Isso ocorre tanto para o isômero syn quanto para o anti.

Essa diferença na carga pode ser entendida pelo fato de que a molécula OM não está parametrizada. Então essas cargas foram obtidas por semelhança com grupos funcionais parametrizados. Por exemplo, todos os grupos metil do OM recebem o mesmo conjunto de parâmetros independentemente de sua vizinhança. O problema maior nesse procedimento é com o conjunto de cargas atômicas. Como, em geral, a molécula tem carga total nula então essa é uma condição que deve ser imposta ao conjunto de cargas que foi obtido por semelhança entre grupos funcionais. Para que essa condição seja satisfeita o que se faz é zerar as cargas dos grupos funcionais. Na figura (4.4) apresentamos os grupos funcionais da molécula OM.

Observa-se na tabela 4.13 que o procedimento de zerar as cargas dos grupos não funciona nessa molécula pois verifica-se que para o conjunto de cargas obtidas com o cálculo teórico a soma das cargas dos grupos dá o negativo da carga do átomo C1, que é um dos átomos que apresenta a maior diferença entre o conjunto de cargas do cálculo teórico e o conjunto do OPLS. No OPLS para que a molécula tenha carga total nula a magnitude da carga nesse átomo deve ser zero.

A mudança que ocorre no dipolo para o conjunto de cargas do OPLS (dipolo do syn maior que o do anti) quando comparado ao dipolo obtido com cálculo quân- 
Tabela 4.12: Análise do conjunto de cargas do OPLS para os dois isômeros.

\begin{tabular}{|c|c|c|c|c|}
\hline Átomo & Gás & & OPLS & \\
\hline & syn & anti & syn & anti \\
\hline $\mathrm{C} 1$ & 0.393 & 0.255 & 0.000 & 0.000 \\
\hline $\mathrm{C} 2$ & -0.585 & -0.508 & -0.115 & -0.115 \\
\hline C3 & 0.746 & 0.716 & 0.500 & 0.500 \\
\hline $\mathrm{O} 4$ & -0.538 & -0.532 & -0.500 & -0.500 \\
\hline C5 & -0.341 & -0.299 & -0.180 & -0.180 \\
\hline $\mathrm{C} 6$ & -0.309 & -0.185 & -0.180 & -0.180 \\
\hline $\mathrm{C} 7$ & -0.326 & -0.238 & -0.180 & -0.180 \\
\hline H8 & 0.156 & 0.164 & 0.115 & 0.115 \\
\hline H9 & 0.085 & 0.076 & 0.060 & 0.060 \\
\hline H10 & 0.085 & 0.076 & 0.060 & 0.060 \\
\hline H11 & 0.084 & 0.079 & 0.060 & 0.060 \\
\hline H12 & 0.083 & 0.060 & 0.060 & 0.060 \\
\hline H13 & 0.087 & 0.059 & 0.060 & 0.060 \\
\hline H14 & 0.087 & 0.059 & 0.060 & 0.060 \\
\hline H15 & 0.080 & 0.074 & 0.060 & 0.060 \\
\hline H16 & 0.133 & 0.069 & 0.060 & 0.060 \\
\hline H17 & 0.080 & 0.074 & 0.060 & 0.060 \\
\hline Dipolo(D) & 2.77 & 3.90 & 3.63 & 3.10 \\
\hline
\end{tabular}




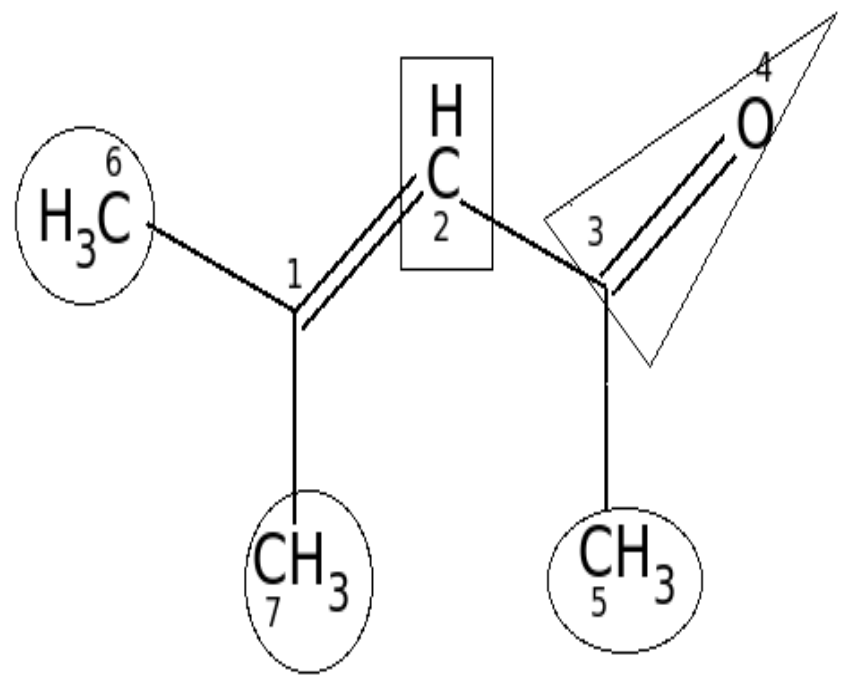

Figura 4.4: Ilustração dos grupos funcionais da molécula OM. Isômero anti.

Tabela 4.13: Análise da carga dos grupos funcionais da molécula OM. Valores obtidos com cálculos quânticos (Gás) e através do campo de força (OPLS)

\begin{tabular}{lcccc}
\hline \hline Grupos & Gás & \multicolumn{3}{c}{ OPLS } \\
\hline & syn & anti & syn & anti \\
$\mathrm{C} \mathrm{H}_{3}$ & -0.052 & -0.007 & 0.000 & 0.000 \\
$\mathrm{C} \mathrm{H}_{3}$ & -0.033 & -0.021 & 0.000 & 0.000 \\
$\mathrm{C} 2 \mathrm{H}$ & -0.429 & -0.344 & 0.000 & 0.000 \\
$\mathrm{C} 3 \mathrm{O} 4$ & 0.208 & 0.184 & 0.000 & 0.000 \\
$\mathrm{C} 5 \mathrm{H}_{3}$ & -0.087 & -0.068 & 0.000 & 0.000 \\
\hline Carga total & -0.393 & -0.256 & 0.000 & 0.000 \\
\hline \hline
\end{tabular}


tico pode ser entendida através de uma análise estrutural da molécula OM. Como os parâmetros do campo de força são os mesmos para os dois isômeros, a única diferença entre eles está na geometria. Observa-se, tabela (4.1), que os isômeros não apresentam muitas diferenças com relação ao comprimento das ligações. A maior diferença observada está na distância entre os átomos $\mathrm{C} 2$ e O4, ela é maior para o isômero syn. Como o momento de dipolo para um par de cargas opostas de magnitude q é definido pela magnitude da carga vezes a distância entre eles $(\vec{p}=q \vec{d})$ tem-se que a contribuição do dipolo devido aos átomos $\mathrm{C} 2$ e O4 para o dipolo total da molécula será maior no isômero syn, o que justifica o fato desse possuir um dipolo total maior no conjunto OPLS. Como a molécula de OM não está parametrizada, nós iremos usar o conjunto de cargas obtidas pelo cálculo quântico que é confiável para a descrição do potencial eletrostático da molécula OM.

Definido os parâmetros do potencial intermolecular nós realizamos simulações para obtermos as estruturas do OM solvatado em água.

O primeiro passo que deve ser feito na simulação é a termalização do sistema (OM + água) para garantirmos que esse esteja em equilíbrio termodinâmico. No processo de termalização foram realizados $15 \times 10^{6}$ passos $\mathrm{MC}$, o que equivale à 30.000 ciclos $\mathrm{MC}$, onde um ciclo se completa quando movemos todas as moléculas da caixa de simulação. Cada ciclo representa uma configuração acessível para o sistema solvatado. Na figura (4.5) apresentamos a energia por molécula versus o número de ciclos para o isômeros syn. Para as termalizações do anti e TS obtivemos gráficos análogos.

Durante o processo de termalização acompanhamos também a equilibração de outras propriedades do sistema como a densidade e a energia de interação solutosolvente.

Após a termalização, realizamos $37.5 \times 10^{6}$ passos $\mathrm{MC}$, o que equivale à 75.000 ciclos MC (75.000 configurações acessíveis ao sistema). Observou-se que a energia por molécula flutua em torno de um valor médio caracterizando que o sistema está num 


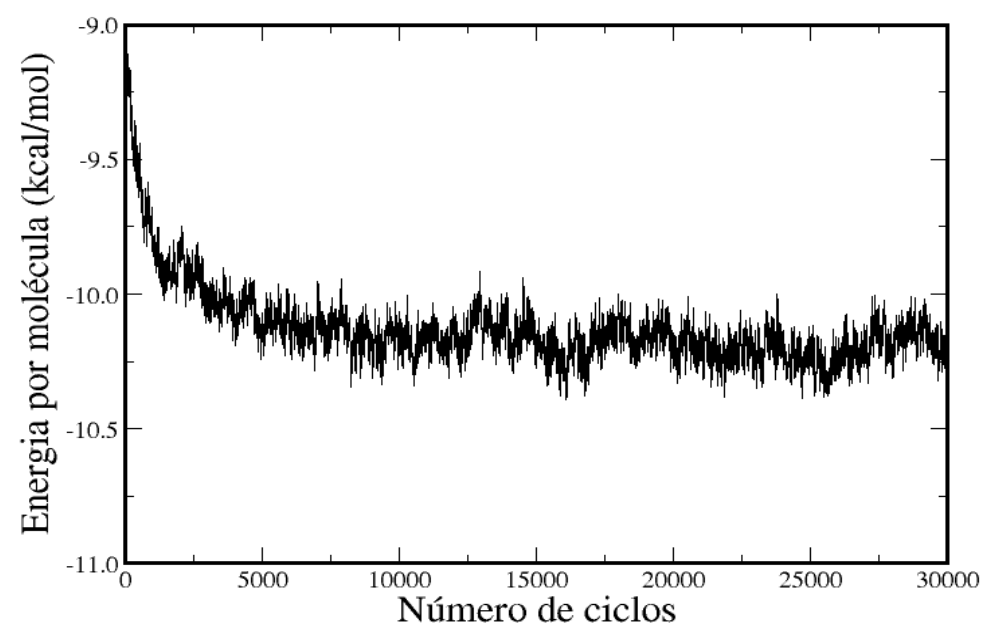

Figura 4.5: Energia por molécula versus o número de ciclos durante o processo de termalização do sistema, isômero syn.

estado de equilíbrio termodinâmico.

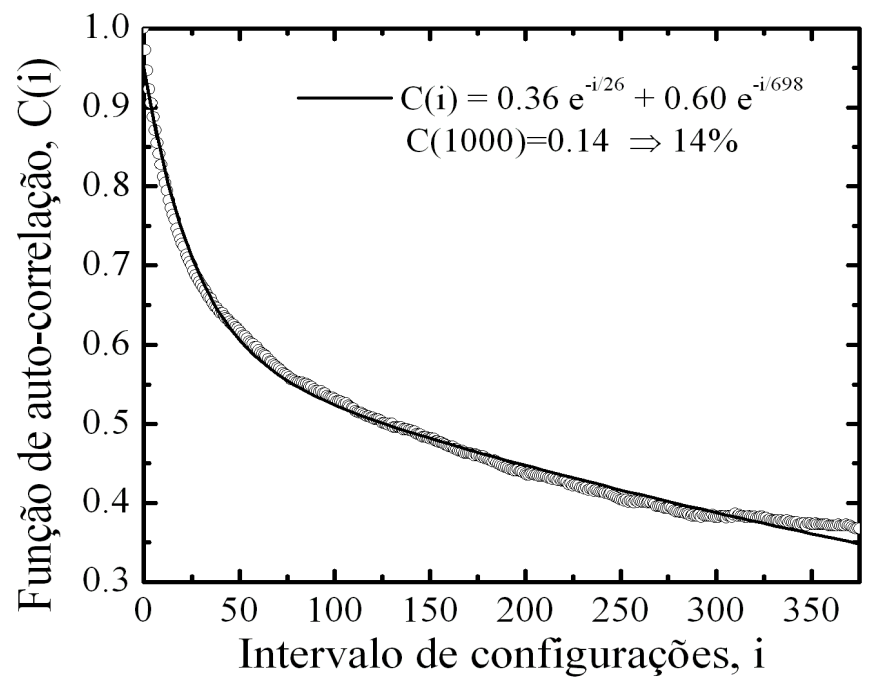

Figura 4.6: Função de auto-correlação estatística da energia potencial de interação por molécula, $U / N$. Isômero syn.

Realizada a simulação computacional do sistema composto pelo soluto e as 
moléculas do solvente, milhares de configurações acessíveis são geradas no MC onde cada configuração possui um conjunto de coordenadas para todos os átomos. O nosso interesse é realizar cálculos QM nessas configurações, mas fazer isso sobre milhares se torna inviável. O que fizemos foi a análise estatística sobre essas configurações de tal forma a reduzir os números de configurações a serem consideradas nos cálculos de QM. O próximo passo então foi encontrar a correlação estatística entre as sucessivas configurações do sistema geradas pela simulação, para isso nós analisamos a função de auto-correlação estatística da energia potencial de interação por molécula $(U / N)$ que nos dá o intervalo de correlação entre as configurações. Na figura 4.6 apresentamos a função de auto-correlação estatística de $U / N$. Essa função foi ajustada por uma função exponencial de decaimento duplo. O ajuste está mostrado na figura (4.6). Substituindo um valor, intervalo $i$ qualquer, é possível calcular através da função ajustada a porcentagem da correlação estatística entre valores de $U / N$ separados pelo intervalo $i$. Como exemplo, mostramos na figura que um intervalo de 1000 configurações apresenta uma correlação estatística de 14\%. Com esse valor de correlação pode-se considerar que as configurações geradas num intervalo de 1000 configurações estão estatisticamente descorrelacionadas. Portanto, os cálculos QM da molécula de OM solvatado em água serão realizados sobre 75 configurações descorrelacionadas.

\subsubsection{Ligação de Hidrogênio}

De maneira a reduzir a região do solvente que será tratada quânticamente a posteriore, um importante aspecto estudado é a distribuição das moléculas do solvente ao redor do soluto, isto é, as camadas de solvatação e as ligações de hidrogênio (LH). A análise dessas quantidades são feitas através da função de distribuição radial de pares(RDF). Existem várias possibilidades para o cálculo da RDF, como a RDF entre o centro de massa do soluto e do solvente e a RDF calculada na mínima distância entre os átomos do soluto e os átomos do solvente, MDDF. Essas possibilidades de cálculos 
de RDF são mais usadas para a obtenção das camadas de solvatação. Na figura (4.7) analisamos a função radial de pares entre o oxigênio do $\mathrm{OM}$ e o oxigênio da água, $G_{O O}(\mathrm{r})$, para os dois isômeros.

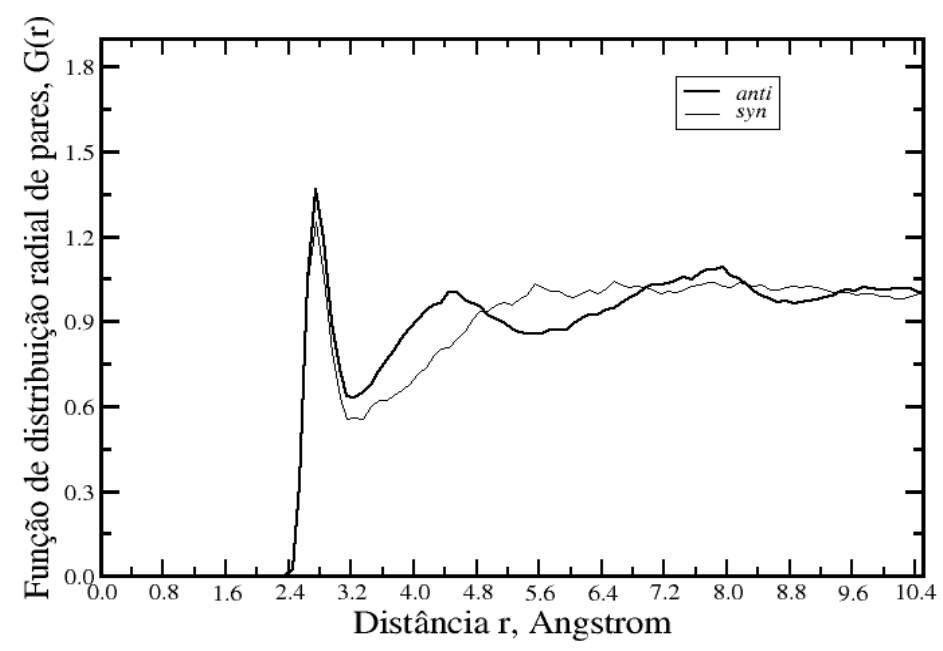

Figura 4.7: Função de distribuição radial de pares, $G_{O O}(\mathrm{r})$.

Na figura 4.7 observa-se que as camadas do anti são mais bem definidas, o que pode ser entendido pela análise do dipolo (o anti possui um maior dipolo). Observase também um pico bem definido nas distâncias entre 2.5 e $3.25 \AA$, da integração desse pico obtém-se 1 molécula de água formando a vizinhança mais próxima para o isômero syn e 2 para o anti. Porém não podemos garantir, somente com a análise da $\mathrm{G}_{O O}(r)$, que essas moléculas estão fazendo LH. Para se estudar quantativamente as ligações de hidrogênio pode-se utilizar um critério energético e geométrico [104, 105. Essa combinação tem obtido êxito na descrição das LHs em líquidos 106, 107. Nesses critérios é necessário definirmos limites para máximos valores de $\mathrm{R}_{O O}, \Theta_{O O H}$ e $\mathrm{E}_{\text {lig }}$. Aqui nós consideramos uma ligação de hidrogênio quando a distância entre o oxigênio do OM e o oxigênio da água, $\mathrm{R}_{O O}$, for menor que $3.25 \AA$, o ângulo entre o oxigênio do OM e o grupo $\mathrm{OH}$ da água, $\Theta_{O O H}$, for menor que $40^{\circ}$ e a energia de ligação, $E_{l i g}$, for 
menor que $-0.01 \mathrm{kcal} / \mathrm{mol}$, ou seja, negativa.

Uma vez definido os critérios das ligações de hidrogênio nós fizemos uma análise estatística das ligações de hidrogênio nos dois isômeros. Na tabela (4.14) listamos o número de ligações formadas com o hidrogênio da água bem como o seu percentual de ocorrência. Observa-se que a maioria das configurações submetidas aos critérios descritos anteriormente, $55 \%$ (syn) e 44.0\% (anti), possuem apenas uma ligação de hidrogênio. Para duas ligações o isômero anti é o que possui um maior percentual de ocorrência e configurações com 3 ligações de hidrogênio foram encontradas apenas para o isômero anti, 1.6\%. Tomando a média entre as configurações analisadas e as que possuem LHs obtém-se que o anti possui, em média, uma ligação de hidrogênio por configuração. Enquanto que o syn possui $0.7 \mathrm{LH}$, isso significa que em média $70 \%$ das configurações apresentaram uma LH. A análise das ligações de hidrogênio nos mostra que a conformação anti apresenta maior habilidade em interagir especificamente com a água. Obtivemos também a energia média das ligações de hidrogênio, $\left\langle E_{L H}\right\rangle$, existentes entre o oxigênio $(\mathrm{O})$ da molécula $\mathrm{OM}$ e os hidrogênios $(\mathrm{H})$ da água. Fizemos isso para os isômeros syn e anti. Nós encontramos valores muito próximos, -4.93 e -4.81 $\mathrm{kcal} / \mathrm{mol}$, para os isômeros syn e anti, respectivamente. Essa melhor estabilização na energia média das ligações de hidrogênio para o isômero syn pode ser entendida pelo fato de que o oxigênio $(\mathrm{O})$, nessa conformação, é mais negativo (ver tabela (4.12)).

\subsubsection{Camadas de Solvatação}

Posteriormente iremos analisar o efeito do solvente no espectro de absorção do OM realizando cálculos quânticos com TD-DFT nas 75 configurações do OM solvatado em água selecionadas da simulação, usando o método QM/MM sequêncial. Uma etapa importante na metodologia sequêncial é a obtenção da distribuição do solvente ao redor do soluto, as camadas de solvatação, que nos permite escolher o tamanho do sistema que será considerado explicitamente no cálculo quântico. 
Tabela 4.14: Análise estatística das ligações de hidrogênio.

\begin{tabular}{llll}
\hline \hline No de LH & \% configurações \\
\hline syn & anti & syn & anti \\
0 & 0 & 36.3 & 25.6 \\
1 & 1 & 55.3 & 44.0 \\
2 & 2 & 8.4 & 28.8 \\
3 & 3 & 0.0 & 1.6 \\
Média & 0.72 & 1.06 \\
\hline \hline
\end{tabular}

As camadas de solvatação foram obtidas através da RDF calculada na mínima distância entre os átomos do soluto e os átomos do solvente, MDDF. Na figura 4.8 apresentamos a MDDF para os dois isômeros. Na MDDF que representa o isômero syn, existe um ombro no primeiro pico com fim em $2.0 \AA$ que caracteriza as moléculas que estão acessíveis às ligações de hidrogênio. Integrando esse ombro obtemos um número de coordenação de 2 moléculas de água. O primeiro pico que termina em 3.9 A, caracteriza a primeira camada de solvatação que contém 34 moléculas de água. O segundo pico entre $3.9 \AA$ e $7.3 \AA$, caracteriza a segunda camada de solvatação e possui 135 moléculas.

Para a MDDF que representa o isômero anti, temos valores semelhantes. Um ombro até 2.2 A que caracteriza as moléculas acessíveis às ligações de hidrogênio. Integrando esse ombro obtemos um número de coordenação de 3 moléculas de água. O primeiro pico até $4.0 \AA$, que caracteriza a primeira camada de solvatação com 34 moléculas e o segundo pico entre até $7.5 \AA$ A, que caracteriza a segunda camada de solvatação e possui 141 moléculas. O limite da caixa possui 250 moléculas de água. Pela análise dos gráficos da figura (4.8) e das camadas de solvatação verificamos que 


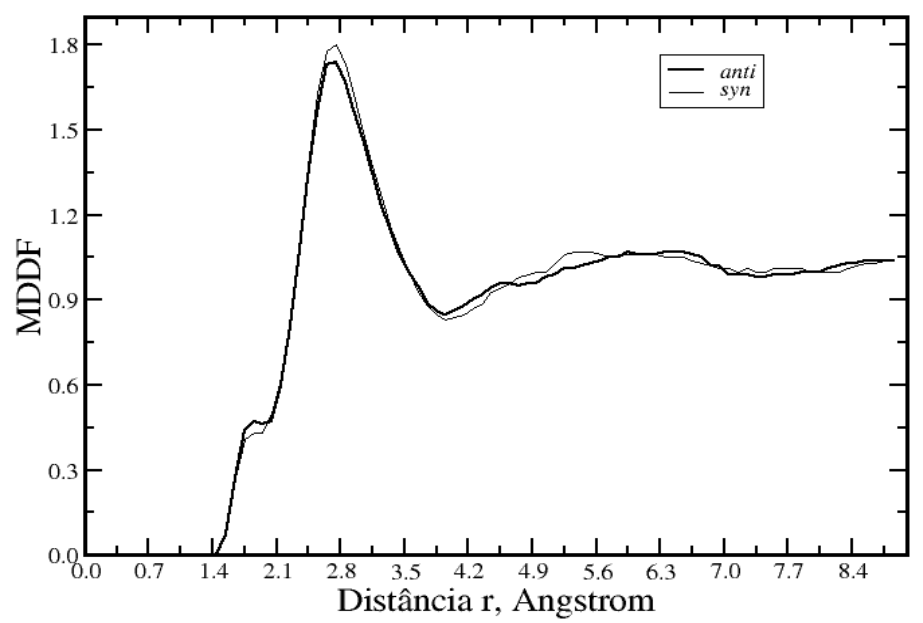

Figura 4.8: RDF de mínima distância entre o $\mathrm{OM}$ e as moléculas de água.

ambos isômeros possuem uma distribuição de água ao seu redor bastante semelhante.

\subsubsection{Incluindo a Polarização do OM em Água}

Como no nosso trabalho fazemos uso da metodologia S-QM/MM onde as simulações clássicas são desacopladas dos cálculos quânticos, a polarização do soluto pelo solvente não surge na simulação. Numa simulação convencional de QM/MM, onde a parte clássica e a parte quântica são realizadas simultaneamente, a polarização mútua entre o soluto e o solvente, que compoem a parte QM, surge naturalmente. Tendo em vista que os efeitos de polarização do soluto pelo solvente são importantes no estudo do solvatocromismo, o nosso grupo desenvolveu um método iterativo usando S-QM/MM para determinar a polarização do soluto na presença do solvente [108]. Esse processo iterativo é ilustrado na figura 4.9 .

O procedimento para a inclusão da polarização do soluto através do processo iterativo ocorre da seguinte forma: realizam-se simulações, usando um conjunto inicial de cargas (em geral obtido para a molécula isolada), é feita a análise estatística e 


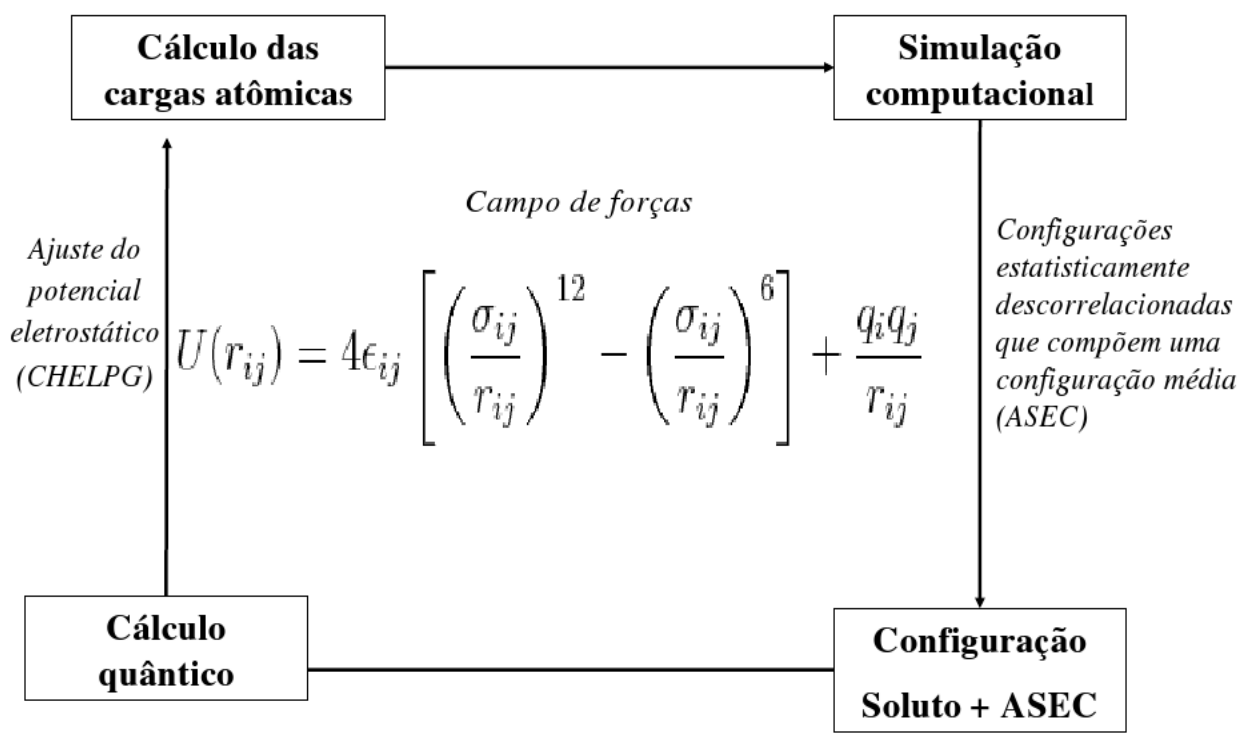

Figura 4.9: Ilustração do processo iterativo para polarização do soluto.

selecionada as configurações descorrelacionadas que compõem uma configuração com uma média eletrostática do solvente (ASEC, configuração composta por 56250 cargas pontuais $=75$ configurações x 250 águas x 3 átomos) [109], onde as moléculas do solvente são consideradas apenas como cargas pontuais. Essa configuração média é gerada superpondo todas as configurações e dividindo as cargas pelo número de configurações utilizadas. A idéia do ASEC é semelhante ao sistema de réplicas muito usado nos estudos de interações de fármacos com proteínas. Em seguida realizamos QM sobre a configuração média para obtermos a densidade eletrônica do soluto na presença do campo eletrostático do solvente, que será representada pelas cargas pontuais, com essas cargas repete-se o procedimento até obtermos um conjunto de cargas convergido.

O conjunto de cargas convergido obtido no processo iterativo para o isômero anti é apresentado na tabela (4.15) juntamente com as cargas obtidas em fase gasosa, com o OPLS e através do modelo contínuo PCM para o solvente. Observa-se que ao incluir a polarização do soluto devido a presença do solvente através do processo iterativo, há um aumento nas cargas atômicas quando comparadas aos conjuntos obtidos nos 
outros procedimentos. Isso pode ser verificado também pelo aumento no momento de dipolo. Observa-se que as cargas obtidas com o modelo contínuo PCM também apresenta um aumento quando comparado ao conjuntos de fase gasosa e OPLS. Isso era esperado pois como a molécula $\mathrm{OM}$ é polar seu campo de reação será não nulo (ver seção 2.6). Na tabela 4.15) o átomo O4 é o que sofre a maior mudança ficando mais negativo no conjunto de cargas obtido com o processo iterativo. O que nos diz que nas configurações do soluto solvatado geradas com esse conjunto de cargas haverá, em média, mais ligações de hidrogênio formadas com a molécula de água quando comparada às configurações geradas com o conjunto de cargas de fase gasosa. Isso será evidenciado na seção 4.2.5 em que fazemos novamente a análise das ligações de hidrogênio. O comportamento apresentado pelo syn e pelo TS é semelhante. No apêndice A apresentamos a tabela com os diferentes conjuntos de cargas para o isômero syn e também para o TS.

Nas figuras 4.10 e 4.11 apresentamos os estágios durante o processo de polarização do OM em água para as conformações syn e anti, respectivamente. Apresentamos também o valor para o dipolo convergido obtido nesse processo.

Após o processo de polarização observa-se que há um aumento no momento de dipolo de aproximadamente $80 \%$, comparativamente ao dipolo da molécula isolada, para os dois isômeros. Os dipolos obtidos pelo processo de polarização do OM em água foram 4.97 e $6.96 \mathrm{D}$, para os isômeros syn e anti, respectivamente. Realizamos o mesmo procedimento para o estado de transição e obtivemos um dipolo de $5.13 \mathrm{D}$. Nas figuras 4.10 e 4.11 apresentamos também o momento de dipolo (linha pontilhada) obtido para os isômeros considerando o solvente através do modelo contínuo polarizável (PCM). Utilizando o método MP2 com as funções base aug-cc-pVDZ e modelando o ambiente de solução aquosa com PCM, observa-se um dipolo de 3.85 D para o syn, $4.01 \mathrm{D}$ para TS e 5.25 D para o anti. Embora esse resultado seja um pouco inferior ao obtido com o método iterativo, ele nos sugere que a utilização do modelo contínuo 
Tabela 4.15: Conjunto das cargas para o isômero anti.

\begin{tabular}{lcccc}
\hline \hline Átomo & Gás & OPLS & PCM & Iterativo \\
\hline C1 & 0.255 & 0.000 & 0.283 & 0.302 \\
C2 & -0.508 & -0.115 & -0.538 & -0.549 \\
C3 & 0.716 & 0.500 & 0.758 & 0.837 \\
O4 & -0.532 & -0.500 & -0.627 & -0.769 \\
C5 & -0.299 & -0.180 & -0.309 & -0.311 \\
C6 & -0.185 & -0.180 & -0.201 & -0.168 \\
C7 & -0.238 & -0.180 & -0.283 & -0.319 \\
H8 & 0.164 & 0.115 & 0.177 & 0.173 \\
H9 & 0.076 & 0.060 & 0.092 & 0.102 \\
H10 & 0.076 & 0.060 & 0.092 & 0.099 \\
H11 & 0.079 & 0.060 & 0.078 & 0.086 \\
H12 & 0.060 & 0.060 & 0.064 & 0.050 \\
H13 & 0.059 & 0.060 & 0.071 & 0.073 \\
H14 & 0.059 & 0.060 & 0.071 & 0.072 \\
H15 & 0.074 & 0.060 & 0.094 & 0.110 \\
\hline \hline & 0.069 & 0.060 & 0.084 & 0.099 \\
Hipolo(D) & 3.90 & 3.10 & 5.25 & 6.96 \\
\hline
\end{tabular}




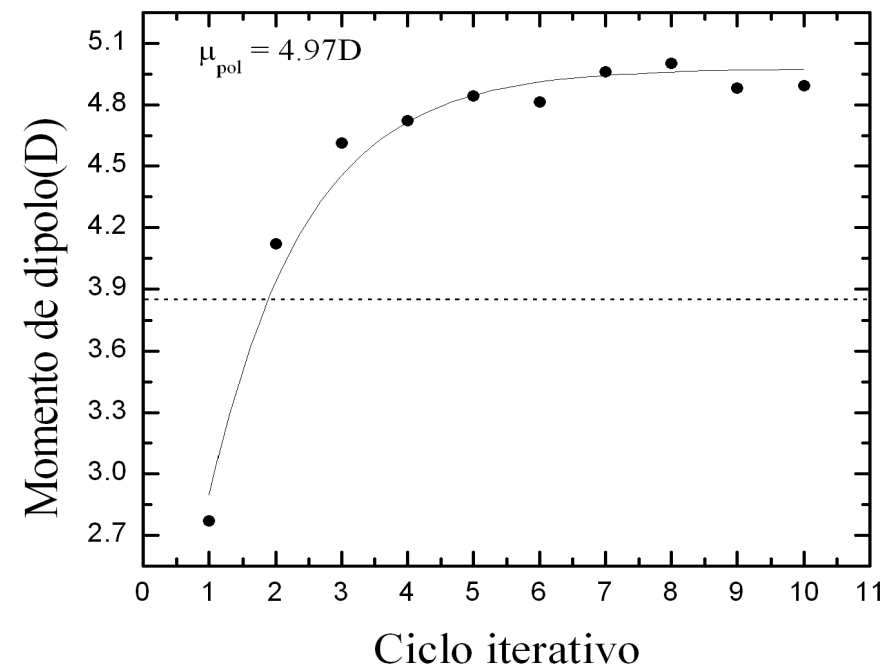

Figura 4.10: Evolução do momento de dipolo com o processo de polarização iterativo. Isômero syn. Linha pontilhada é o valor do dipolo obtido com o modelo contínuo de solvente PCM.

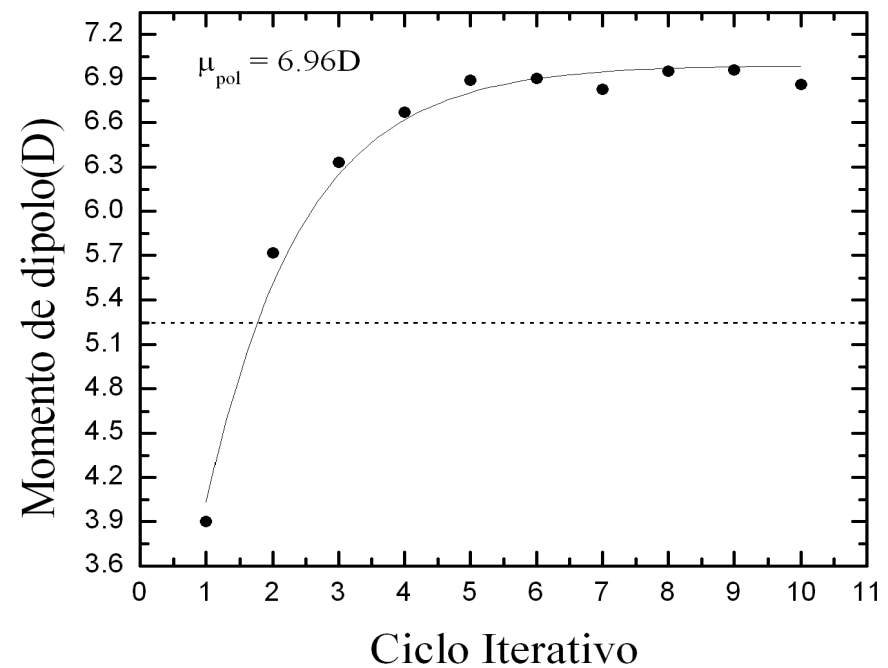

Figura 4.11: Evolução do momento de dipolo com o processo de polarização iterativo. Isômero anti. Linha pontilhada é o valor do dipolo obtido com o modelo contínuo de solvente PCM. 
é uma aproximação razoável para obtenção dos momentos de multipolo de moléculas em solução quando o método iterativo acarretar um alto custo computacional. Note que no processo iterativo foram necessárias 10 simulações e 10 cálculos quânticos para cada isômero, o que torna um procedimento com alto custo computacional comparado ao cálculo realizado com o modelo contínuo PCM.

\subsubsection{Ligação de Hidrogênio: Soluto Polarizado}

Uma vez obtida a polarização do soluto nós fizemos novamente a análise estatística das ligações de hidrogênio pois agora com um novo conjunto de cargas o solvente se redistribui ao redor do soluto. Na figura (4.12) apresentamos a função radial de pares entre o oxigênio do OM e o oxigênio da água, $G_{O O}(\mathrm{r})$, para os dois isômeros. Observa-se na figura um pico bem definido nas distâncias entre 2.5 e $3.25 \AA$, esse pico é mais pronunciado (comparado ao pico da G(r) obtida sem a polarização) mostrando uma maior densidade de água em torno do oxigenio do OM. Da integração desse pico obtém-se 2 moléculas de água formando a vizinhança mais próxima para o isômero syn e 3 para o anti. Portanto, quando a polarização do soluto pelo solvente é levada em consideração a interação entre eles é aumentada. Aqui mantivemos o mesmo critério definido anteriormente (ver seção 4.2.2).

Refazendo a análise estatística das ligações de hidrogênio formadas com as moléculas de água, tabela 4.16), observamos que há, em geral, um aumento na ocorrência das configurações em que existe ligação de hidrogênio quando comparamos com os resultados obtidos sem incluir a polarização do soluto (ver tabela (4.14)). Esse aumento na ocorrência das ligações de hidrogênio pode ser entendido pela tabela 4.15 em que se observa um aumento nas cargas atômicas da molécula OM quando a polarização do soluto é levada em conta através do processo iterativo. Observa-se também que em média o isômero syn possui uma ligação de hidrogênio com a água e o anti possui uma ligação e meia. As energias médias das LH's obtidas depois do processo 


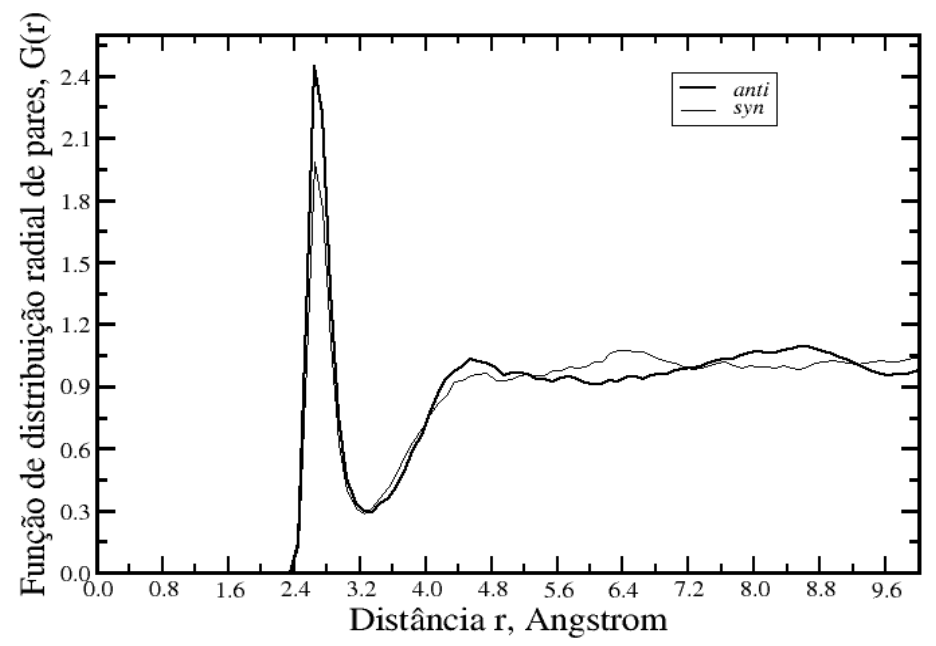

Figura 4.12: Função de distribuição radial de pares, $G_{O O}(\mathrm{r})$.

Tabela 4.16: Análise estatística das ligações de hidrogênio.

\begin{tabular}{lllll}
\hline \hline No de LH & \% configurações \\
\hline syn & anti & syn & anti \\
0 & 0 & 22.1 & 0.4 \\
1 & 1 & 42.7 & 42.7 \\
2 & 2 & 33.5 & 55.3 \\
3 & 3 & 1.7 & 1.6 \\
Média & 1.15 & 1.58 \\
\hline \hline
\end{tabular}


de polarização para os dois isômeros foram -7.33 e $-8.22 \mathrm{kcal} / \mathrm{mol}$ para o syn e anti, respectivamente. Observa-se que há um acréscimo na energia da ligação de hidrogênio quando comparada com a energia obtida com as cargas de fase gasosa (soluto isolado). Esse acréscimo deve-se ao fato de que nesse novo conjunto de cargas o oxigênio, que recebe a ligação de hidrogênio, está mais negativo (ver tabela 4.15, para o syn ver apêndice).

\subsubsection{Camadas de Solvatação: Soluto Polarizado}

Revisitando as camadas de solvatação do OM em água após o processo de polarização. Na figura (4.13) apresentamos a RDF de mínima distância para os isômeros syn e anti. Na MDDF que representa o isômero syn, a diferença maior, com relação a figura 4.8), é o aparecimento do ombro mais pronunciado finalizando em $2.2 \AA$ que caracteriza as moléculas acessíveis às ligações de hidrogênio. A integração desse ombro dá um número de coordenação igual a 3 moléculas de água. O primeiro pico entre até $4.0 \AA$, caracteriza a primeira camada de solvatação e a integração desse pico dá 35 moléculas de água. O segundo pico entre até $7.3 \AA$ caracteriza a segunda camada de solvatação e apresenta uma quantidade de 137 moléculas de água.

Para a MDDF que representa o anti, observa-se também um aumento no ombro, quando comparado à figura (4.8). A integração desse ombro até $2.2 \AA$, corresponde as moléculas acessíveis às ligações de hidrogênio e apresenta uma quantidade de 4 moléculas de água. O primeiro pico entre até $4.0 \AA$, caracteriza a primeira camada de solvatação e possui uma quantidade de 35 moléculas de água. O segundo pico até 7.5 A, caracteriza a segunda camada de solvatação sendo um total de 142 moléculas de água nessa camada. Portanto, verificamos que, assim como no caso para o soluto não polarizado, ambos isômeros possuem uma distribuição de água ao seu redor bastante semelhante. 


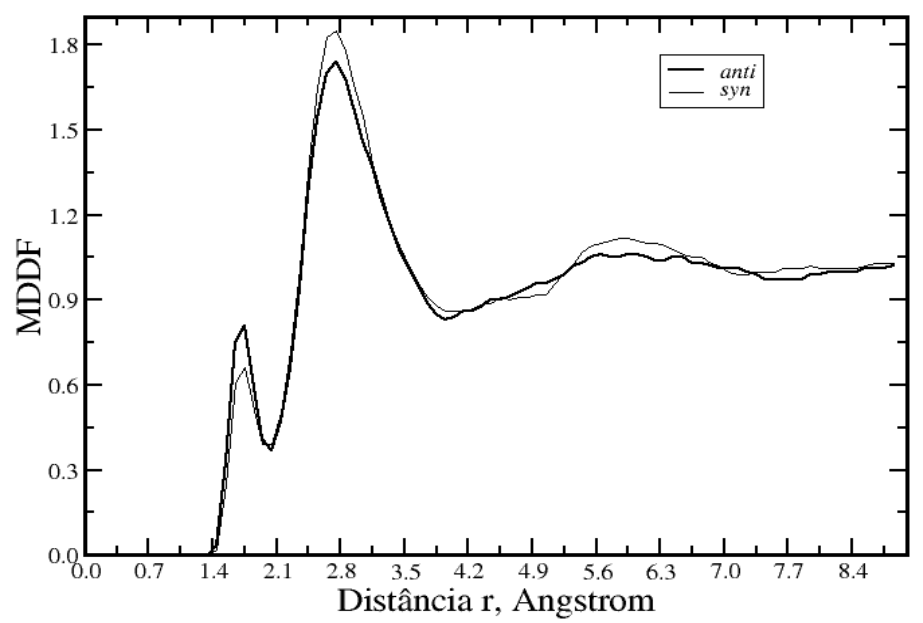

Figura 4.13: RDF de mínima distância entre o OM e as moléculas de água. Isômero syn.

\subsubsection{Análise Conformacional}

Para obter a estabilidade conformacional do OM em solução aquosa calculamos a variação de energia livre de Gibbs associada à mudança syn-anti. A energia livre associada a essa mudança pode ser obtida através do ciclo termodinâmico mostrado na figura 4.14 que garante a seguinte relação

$$
\Delta G_{\text {syn } \rightarrow \text { anti }}(\text { água })=\Delta G_{\text {syn } \rightarrow a n t i}(\text { gás })+\left[\Delta G_{\text {anti }}-\Delta G_{\text {syn }}\right]
$$

onde $\Delta G_{\text {syn } \rightarrow \text { anti }}\left(\right.$ gás) é obtido da tabela (4.6). Os termos $\Delta G_{a n t i}$ e $\Delta G_{s y n}$ são as energias livres de solvatação de cada uma das conformações em solução aquosa. A energia livre de cada conformação $\left(\Delta G_{\text {anti }}\right.$ e $\left.\Delta G_{\text {syn }}\right)$ em solução aquosa foi obtida por dois procedimentos : i) através de cálculo quântico usando modelo contínuo para o solvente (PCM) (ver seção 2.6) ii) considerando o modelo discreto para o solvente usando método Monte Carlo e Teoria de Perturbação Termodinâmica (TPT) (detalhada na seção 3.2.5), que estão implementados no programa DICE. 


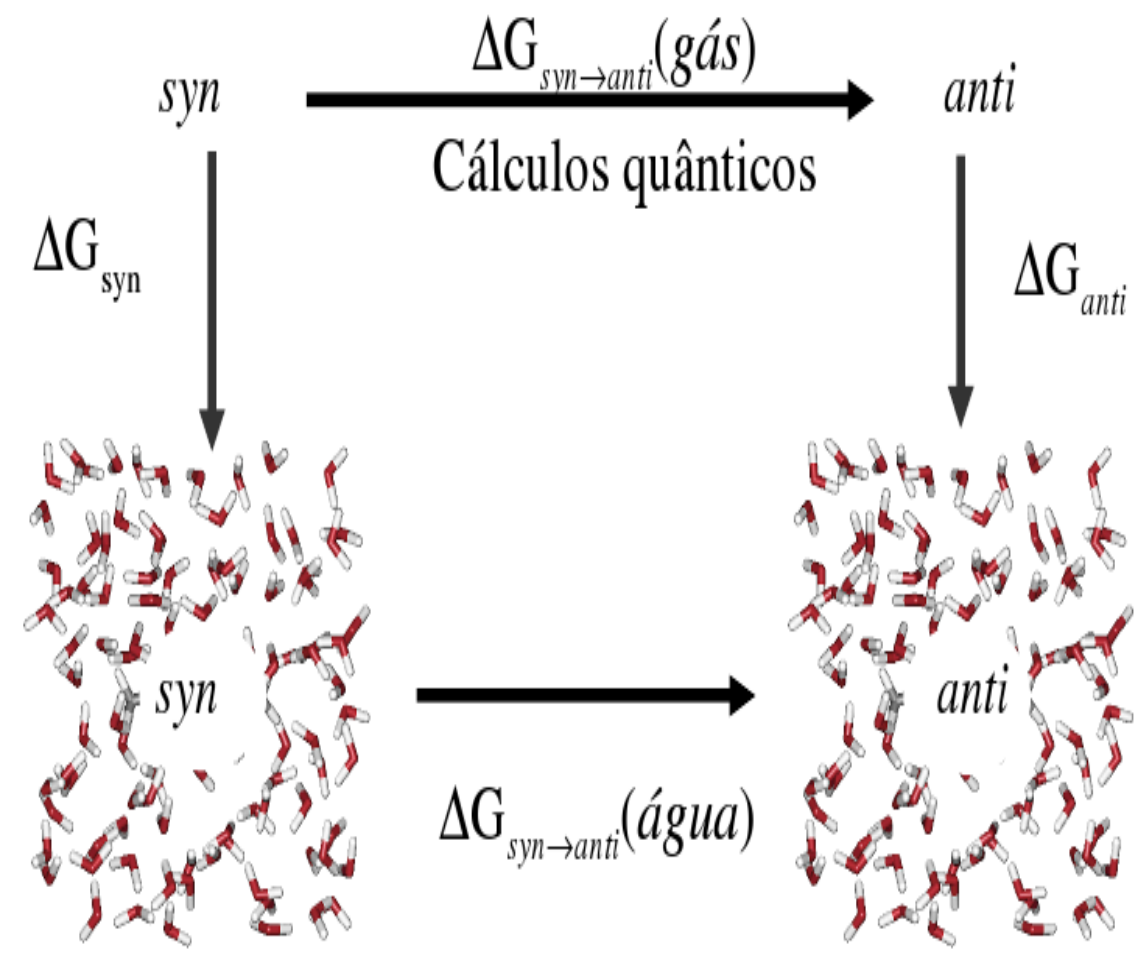

Figura 4.14: Ilustração do ciclo termodinâmico para o cálculo da variação de energia livre da mudança conformacional syn $\rightarrow$ anti em solução aquosa. 
Para obter a energia livre de solvatação considerando o modelo contínuo para o solvente nós realizamos cálculo quântico no nível B3LYP/6-311 $++\mathrm{G}^{* *}$, o solvente considerado foi a água $(\epsilon=78.39)$. Na tabela (4.17) apresentamos as contribuições para a energia livre de solvatação em cada conformação, aqui o solvente é descrito apenas pela sua constante dielétrica, $\epsilon$.

Tabela 4.17: Tabela com as contribuições para a energia livre de solvatação em água (kcal/mol) obtidas considerando o solvente como um meio contínuo (PCM).

\begin{tabular}{lccc}
\hline \hline Contribuição & $\Delta G_{\text {syn }}$ & $\Delta G_{T S}$ & $\Delta G_{\text {anti }}$ \\
\hline Eletrostática & -5.01 & -5.82 & -6.41 \\
van der Waals & -10.57 & -10.49 & -10.63 \\
Cavitação & 20.99 & 20.94 & 20.45 \\
\hline$\Delta G_{\text {total }}$ & 4.63 & 3.62 & 2.46 \\
\hline$\Delta G_{\text {syn } \rightarrow T S}$ & -1.01 & & \\
$\Delta G_{\text {syn } \rightarrow \text { anti }}$ & -2.17 & & \\
\hline \hline
\end{tabular}

Na tabela (4.17) observa-se que o isômero anti apresenta uma maior contribuição eletrostática para a energia livre de solvatação em água. Isso era esperado, pois esse isômero possui um maior momento de dipolo (ver tabela (4.8)). As demais contribuições são equivalentes nos dois isômeros. O que não parece razoável é um valor positivo para a energia livre de solvatação $\left(\Delta G_{t o t a l}\right)$ em água, pois como a molécula é polar era de se esperar que o modelo contínuo para o solvente fosse capaz de descrever, relativamente bem, as interações existente entre o soluto e o solvente. Esses resultados também estão em discrepância com a evidência experimental de que essas moléculas são solúveis em água.

Para obter a energia livre de solvatação usando o modelo discreto para o solvente 
nós realizamos simulações no ensemble NPT, à $298 \mathrm{~K}$ e $1 \mathrm{~atm}$. O sistema considerado foi 1 OM com 1000 águas. A interação entre o OM e a água é descrita pelo potencial de Lennard-Jones e Coulomb (LJC), equação (3.31). Os parâmetros $\epsilon$ e $\sigma$ do potencial de Lennard-Jones foram os mesmos usados anteriormente e o conjunto de cargas que representam o termo coulombiano advém do processo de polarização (descrito na seção 3.2.4). O modelo usado para a água foi o mesmo, SPC.

O cálculo da energia livre foi feito através de um processo hipotético em que sumimos com a molécula do soluto que está dentro da caixa com as moléculas de água, isto é feito usando TPT. O processo de sumir com a molécula é dividido em três estágios. Separadamente, zeramos os três parâmetros do potencial LJC, $q_{i}, \epsilon_{i}$ e $\sigma_{i}$. Primeiro, o potencial de Coulomb vai sendo aniquilado a medida que zeramos as cargas atômicas $q_{i}$. O fator de escala usado para zerar as cargas foi $\lambda=1.00,0.95,0.90,0.80,0.70,0.55,0.40,0.20,0.00$. Usando a técnica de amostragem de passo duplo (double wide) [110 na simulação realizada com $\lambda_{i}$, é feita perturbações simultaneamente para $\lambda_{i-1}$ e $\lambda_{i+1}$. Nesse primeiro estágio, nós realizamos 4 simulações com $\lambda_{i}=0.95,0.80,0.55$ e 0.20 , os valores calculados para $\Delta G^{q}$ em cada intervalo $\lambda_{i}$ são apresentados na tabela 4.18. O segundo estágio é zerar o parâmetro atômico $\epsilon_{i}$ do potencial LJ, porém pela equação (3.31) vemos que se $\epsilon$ for zero todo o potencial de LJ será nulo e isso não é desejável, pois queremos obter também a contribuição do termo de repulsão, então fazemos com que $\epsilon$ seja próximo de zero mas não nulo, o fator de escala usado foi $\lambda=1.00,0.75,0.50,0.25,0.01$. Devido a amostragem de passo duplo, nesse segundo estágio realizamos apenas duas simulações com $\lambda_{i}=0.75$ e 0.25 . Os valores calculados para $\Delta G^{\epsilon}$ em cada intervalo $\lambda_{i}$ são apresentados na tabela 4.19). No terceiro estágio, desaparecemos com o termo repulsivo do potencial zerando os parâmetros atômicos $\sigma_{i}$, o fator de escala usado foi $\lambda=1.00,0.75,0.50,0.25,0.00$. Nesse caso não usamos a amostragem de passo duplo, devido a grande variação de energia do termo quando se cresce $\sigma$. Daí são necessárias 
4 simulações com $\lambda_{i}=1.00,0.75,0.50,0.25$. Os valores calculados para $\Delta G^{\sigma}$ em cada intervalo $\lambda_{i}$ são apresentados na tabela $(4.20)$. Em todos os estágios foram realizados $9 \times 10^{7}$ passos MC (equivale a 90.000 ciclos) no processo de termalização e $15 \times 10^{7}$ passos MC (equivale a 150.000 ciclos) na simulação com o sistema em equilíbrio.

Na tabela 4.18 apresentamos a energia livre necessária para desaparecermos com o termo Coulombiano, observa-se que o isômero anti apresenta a necessidade de uma maior energia. Isso é decorrente do maior dipolo do anti.

Tabela 4.18: Tabela com a variação de energia livre $(\mathrm{kcal} / \mathrm{mol})$ necessária para desaperecer com a contribuição eletrostática do $\mathrm{OM}$ em água.

\begin{tabular}{lcccc}
\hline \hline$\lambda_{i}$ em q & $\lambda_{j}$ em q & $\Delta G_{\text {syn }}^{q}$ & $\Delta G_{T S}^{q}$ & $\Delta G_{\text {anti }}^{q}$ \\
\hline 1.00 & 0.95 & 1.42 & 1.42 & 1.75 \\
0.95 & 0.90 & 1.33 & 1.33 & 1.65 \\
0.90 & 0.80 & 2.38 & 2.50 & 3.00 \\
0.80 & 0.70 & 2.07 & 2.11 & 2.56 \\
0.70 & 0.55 & 2.34 & 2.28 & 3.02 \\
0.55 & 0.40 & 1.53 & 1.58 & 2.02 \\
0.40 & 0.20 & 1.20 & 1.21 & 1.62 \\
0.20 & 0.00 & 0.44 & 0.36 & 0.58 \\
\hline Total do estágio 1 & & 12.71 & 12.79 & 16.20 \\
\hline \hline
\end{tabular}

Para desaparecermos com o termo atrativo do potencial de LJ, tabela 4.19, observa-se que a energia gasta é praticamente igual para as três conformações.

Com relação ao termo repulsivo do potencial LJ, que nos dá a energia necessária para abrirmos uma cavidade dentro da caixa contendo moléculas de água, observa-se, tabela (4.20), que as conformações apresentam energias próximas e pode ser entendido 
Tabela 4.19: Tabela com a variação de energia livre $(\mathrm{kcal} / \mathrm{mol})$ necessária para desaperecer com a contribuição de van der Waals do OM em água.

\begin{tabular}{lcccc}
\hline \hline$\lambda_{i}$ em $\epsilon, \mathrm{q}=0$ & $\lambda_{j}$ em $\epsilon, \mathrm{q}=0$ & $\Delta G_{\text {syn }}^{\epsilon}$ & $\Delta G_{T S}^{\epsilon}$ & $\Delta G_{\text {anti }}^{\epsilon}$ \\
\hline 1.00 & 0.75 & 1.73 & 1.71 & 1.74 \\
0.75 & 0.50 & 1.98 & 1.95 & 1.99 \\
0.50 & 0.25 & 2.37 & 2.32 & 2.42 \\
0.25 & 0.01 & 2.91 & 3.01 & 3.11 \\
\hline Total do estágio 2 & & 8.99 & 8.99 & 9.26 \\
\hline \hline
\end{tabular}

olhando a figura (4.2) onde vemos que não há uma mudança muito grande no volume do OM quando esse assume cada uma das três conformações.

Tabela 4.20: Tabela com a variação de energia livre $(\mathrm{kcal} / \mathrm{mol})$ necessária para desaparecer com o termo repulsivo, cavitação do OM em água.

\begin{tabular}{lcccc}
\hline \hline$\lambda_{i} \mathrm{em} \epsilon, \mathrm{q}=0$ & $\lambda_{j}$ em $\epsilon, \mathrm{q}=0$ & $\Delta G_{\text {syn }}^{\sigma}$ & $\Delta G_{T S}^{\sigma}$ & $\Delta G_{\text {anti }}^{\sigma}$ \\
\hline 1.00 & 0.75 & -2.79 & -2.54 & -2.17 \\
0.75 & 0.50 & -2.62 & -2.13 & -2.57 \\
0.50 & 0.25 & -1.97 & -2.25 & -2.29 \\
0.25 & 0.01 & -0.97 & -0.95 & -0.97 \\
\hline Total do estágio 3 & & -8.35 & -7.87 & -8.00 \\
\hline \hline
\end{tabular}

A variação de energia livre total para desaparecer com o OM dentro da caixa de simulação com as moléculas de água é dada pela soma da energia obtida em cada um dos três estágios. Iremos representar esse termo por $\Delta G_{\times \rightarrow 0}$, onde $\times$ será o soluto, que neste trabalho é a molécula de OM. Portanto, podemos obter a energia livre de 
solvatação do OM em água. Essa energia será dada pelo negativo da energia livre para aniquilação do soluto, $\Delta G_{\times \rightarrow 0}$. Esses resultados são apresentados na tabela 4.21 .

Tabela 4.21: Valores da variação de energia livre (kcal/ $\mathrm{mol}$ ) obtida em cada estágio, a energia livre total para desaparecer com o $\mathrm{OM}\left(\Delta G_{\times \rightarrow 0}\right)$ e a energia livre de solvatação aquosa do OM $\left(\Delta G_{\times}\right)$nos diferentes isômeros. Apresentamos também as diferenças da energia livre associadas às mudanças conformações.

\begin{tabular}{lccc}
\hline \hline Contribuição & $\Delta G_{\text {syn }}$ & $\Delta G_{T S}$ & $\Delta G_{\text {anti }}$ \\
\hline Eletrostática & 12.71 & 12.79 & 16.20 \\
van der Waals & 8.99 & 8.99 & 9.26 \\
Cavitação & -8.35 & -7.87 & -8.00 \\
\hline$\Delta G_{\times \rightarrow 0}$ & 13.35 & 13.91 & 17.46 \\
$\Delta G_{\times}$ & -13.35 & -13.91 & -17.46 \\
\hline$\Delta G_{\text {syn } \rightarrow T S}$ & -0.56 & & \\
$\Delta G_{\text {syn } \rightarrow \text { anti }}$ & -4.11 & & \\
\hline \hline
\end{tabular}

Aqui, $\Delta G_{s y n \rightarrow T S}$ é obtido pela diferença na energia livre entre as duas conformações, $\Delta G_{s y n \rightarrow T S}=\Delta G_{T S}-\Delta G_{s y n}$, o mesmo ocorre para $\Delta G_{s y n \rightarrow a n t i}$. Observa-se na tabela (4.21) que a contribuição soluto-solvente para a estabilização do isômero anti é maior, observamos também que a barreira energética, $\Delta G_{s y n \rightarrow T S}$, sofre uma diminuição quando comparada com o valor obtido para os isômeros isoladamente (ver tabela (4.6)). Comparando os resultados da tabela (4.21) com os resultados obtidos anteriormente usando modelo contínuo para o solvente (ver tabela (4.17)), percebese que de fato valores positivos para a energia livre de solvatação do OM em solução aquosa não são razoáveis. O que nos leva a concluir que, para o nosso sistema, o modelo contínuo não descreve bem a energia livre de solvatação. Sendo, portanto, necessário a inclusão de interações específicas soluto-solvente, o que é conseguido através do modelo 
discreto.

De posse dos valores de fase gasosa para a energia livre, obtida com diferentes níveis de cálculo quântico, tabela (4.6), e dos valores para a energia livre em solução aquosa, tabela 4.21). Nós podemos, através da equação 4.4), obter a energia livre associada a mudança syn $\rightarrow$ anti. Uma vez obtida a energia livre associada a essa mudança nós podemos então obter as populações (ou concentrações) associadas a cada conformação da mesma forma que foi feito em fase gasosa na seção 4.1.1.

Na tabela 4.22 apresentamos a população do isômero anti em solução aquosa juntamente com a energia livre associada a mudança conformacional syn $\rightarrow$ anti. Na tabela 4.22 também é apresentado o valor da barreira energética $\left(\Delta G_{s y n \rightarrow T S}\right)$.

Tabela 4.22: Tabela com os valores da variação de energia livre $(\mathrm{kcal} / \mathrm{mol})$ associada as mudanças syn $\rightarrow$ anti e syn $\rightarrow T S$, com os valores de fase gasosa obtidos pelos diferentes métodos.

\begin{tabular}{lccc}
\hline \hline Método & $\Delta G_{\text {syn } \rightarrow T S}$ & $\Delta G_{\text {syn } \rightarrow \text { anti }}$ & População (\%) anti \\
\hline B3LYP/6-31+G* & 5.37 & -1.91 & 96 \\
B3LYP/aug-cc-pVDZ & 6.24 & -2.11 & 97 \\
MP2/aug-cc-pVDZ & 3.01 & -2.77 & 99 \\
MP2/aug-cc-pVDZ//B3LYP/6-31+G* & 3.54 & -1.69 & 95 \\
\hline \hline
\end{tabular}

Na tabela 4.22 observa-se que o isômero anti é a conformação mais estável do OM em solução aquosa e que o método MP2 é o que apresenta menor variação para energia livre. Observa-se também que em todos os níves de cálculos quânticos (necessários para obter a energia livre da molécula isolada) a população do isômero anti em solução é maior do que $95 \%$ e que a água provoca uma diminuição na barreira energética. Através do estudo feito nessa seção também podemos conluir que o modelo contínuo PCM não descreve bem a solvatação aquosa do OM. 
Na seção seguinte apresentamos o efeito do solvente no espectro de absorção do OM.

\subsubsection{Espectro de Absorção}

Em solução aquosa, o OM apresenta um deslocamento da banda espectral (deslocamento água $\rightarrow$ iso-octano) de $-2150 \pm 350 \mathrm{~cm}^{-1}[9 \mid$, ou seja, o efeito do solvente ocorre no sentido de diminuir a energia de absorção. Essa transição mais intensa é do tipo $\pi-\pi^{*}$. Nós realizamos cálculos das energias eletrônicas de absorção considerando o solvente como um meio contínuo, PCM, onde é levado em conta a interação eletrostática e tratando o solvente com o modelo discreto onde pode ser incluso tanto a interação eletrostática como também a interação entre os seus orbitais.

O estudo do efeito do solvente no espectro de absorção do OM com o modelo discreto para o solvente, foi feito usando o método sequencial QM/MM, primeiro a parte clássica e posteriormente a parte quântica. Aqui, o cálculo quântico foi feito sobre as configurações descorrelacionadas obtidas anteiromente, total de 75 configurações. Para obtermos as energias eletrônicas de absorção nós realizamos QM usando o método TD-DFT onde foi utilizado dois funcionais de densidade, B3LYP e BHandHLYP, que incluem a correlação eletrônica de maneira diferente. A função base utilizada foi 6$311++\mathrm{G}^{* *}$ que além de descrever bem o espectro de fase gasosa apresenta um bom compromisso com o custo computacional.

As abreviações na tabela 4.23 tem os seguintes significados : PCM significa que o solvente é tratado como um meio contínuo; ASEC é uma configuração média onde o solvente é tratado como cargas pontuais (PC); LH significa que foi considerado 1 ligação de hidrogênio; $n \mathrm{PC}$ diz quantas moléculas de água, $n$, foram tratadas como cargas pontuais; $m \mathrm{~W}$ diz quantas moléculas de água, $m$, foram tratadas explicitamente. Então, por exemplo, a abreviação $\mathrm{S}+8 \mathrm{~W}+242 \mathrm{PC}$ significa que o sistema em que é realizado o cálculo quântico é composto pelo soluto $\mathrm{S}(\mathrm{OM})$ mais 8 moléculas 
explícitas de água mais 242 moléculas de água consideradas como cargas pontuais.

Tabela 4.23: Efeito do solvente na energia de absorção $\left(\mathrm{cm}^{-1}\right)$ da transição $\pi-\pi^{*}$ do OM. Os valores foram obtidos com TD/DFT usando o conjunto de funções base $6-311++\mathrm{G}^{* *}$. $\Delta E_{\text {água }}=E_{\text {água }}-E_{\text {gás }}$

\begin{tabular}{lrlll}
\hline \hline Modelo & \multicolumn{2}{c}{$\Delta E_{\text {agua }}($ syn $)$} & \multicolumn{2}{c}{$\Delta E_{\text {água }}($ anti $)$} \\
\hline & B3LYP & BHandHLYP & B3LYP & BHandHLYP \\
$\mathrm{S}+\mathrm{PCM}$ & -1510 & -1551 & -1855 & -2088 \\
$\mathrm{~S}+\mathrm{ASEC}$ & -1645 & -1615 & -2385 & -2526 \\
$\mathrm{~S}+\mathrm{LH}+250 \mathrm{PC}$ & $-2129 \pm 59$ & $-2021 \pm 60$ & $-3025 \pm 85$ & $-3022 \pm 70$ \\
$\mathrm{~S}+8 \mathrm{~W}+242 \mathrm{PC}$ & $-3039 \pm 112$ & $-2644 \pm 71$ & $-4077 \pm 92$ & $-3979 \pm 83$ \\
$\mathrm{~S}+12 \mathrm{~W}+238 \mathrm{PC}$ & $-3187 \pm 85$ & $-2777 \pm 72$ & $-4356 \pm 101$ & $-4156 \pm 87$ \\
$\mathrm{~S}+14 \mathrm{~W}+236 \mathrm{PC}$ & $-3256 \pm 83$ & $-2810 \pm 73$ & $-4459 \pm 115$ & $-4232 \pm 88$ \\
\hline \hline
\end{tabular}

Os valores teóricos para o deslocamento nas energias de absorção do OM em água são apresentados na tabela 4.23. Esse deslocamento é obtido pela diferença das energias eletrônicas em água e em fase gasosa $\left(\Delta E_{\text {água }}=E_{\text {água }}-E_{\text {gás }}\right)$. Para as energias eletrônicas de fase gasosa utilizamos as energias obtidas nas tabelas (4.9) para o funcional B3LYP e 4.24 para o funcional BHandHLYP com o conjunto de funções base 6-311 $++\mathrm{G}^{* *}$. Observa-se na tabela 4.23 que tanto o modelo contínuo para o solvente quanto o ASEC, modelo em que o solvente é tratado somente como cargas pontuais, descrevem a tendência na diminuição da energia. Quando levamos em conta, além da interação eletrostática, uma interação específica entre o soluto e o solvente, ligações de hidrogênio (LH), observamos que há um deslocamento maior, comparado aos modelos que não incluem interações específicas, na tendência de diminuir a energia eletrônica. 
Esse resultado nos deu indícios de que um fator importante na descrição do efeito do solvente nas energias de absorção do OM seria a interação explícita do soluto com o solvente. Desta forma, realizamos cálculos onde consideramos explicitamente, 8, 12 e 14 águas. Nós não consideramos a primeira camada de solvatação completa (aproximadamente 35 moléculas de água) explicitamente, porque o custo computacional para calcular as energias eletrônicas em cada conformação se torna muito alto. Lembrando que esse cálculo deve ser feito para cada uma das 75 configurações do isômero syn e nas 75 configurações do isômero anti. Portanto, tratamos apenas algumas moléculas mais próximas de maneira explícita e as demais consideramos como sendo cargas pontuais. Como até o limite da caixa de simulação contém 250 águas, as demais que não foram consideradas explicitamente nos cálculos entram como cargas pontuais para completar a quantidade de águas contidas na caixa.

Observa-se na tabela 4.23 que o aumento de águas explícitas de 12 para 14 provoca uma mudança na energia para o isômero syn de $69 \mathrm{~cm}^{-1}$ e $33 \mathrm{~cm}^{-1}$ para os funcionais B3LYP e BHandHLYP, respectivamente, o que indica a convergência do nosso resultado. Essa convergência também pode ser observada pela figura 4.15 em que é apresentado o comportamento do deslocamento solvatocrômico $\left(\mathrm{cm}^{-1}\right)$ versus o número de águas explícitas. No modelo com 14 moléculas de água explícitas há um total de 194 elétrons, caracterizando um tamanho bastante razoável para o sistema. Aqui, vale lembrar que, os valores para o deslocamento espectral, considerando o modelo discreto, são obtidos através de uma média estatística sobre as 75 configurações descorrelacionadas, portanto, o erro que apresentamos para o valor teórico é um erro estatístico.

No cálculo da estabilidade do OM em solução aquosa observamos que o anti é mais estável e em fase gasosa a conformação syn é favorecida, portanto, o deslocamento no espectro de absorção terá duas contribuições com relação ao efeito do solvente : o efeito na mudança conformacional syn $\rightarrow$ anti e o efeito na estrutura eletrônica. 


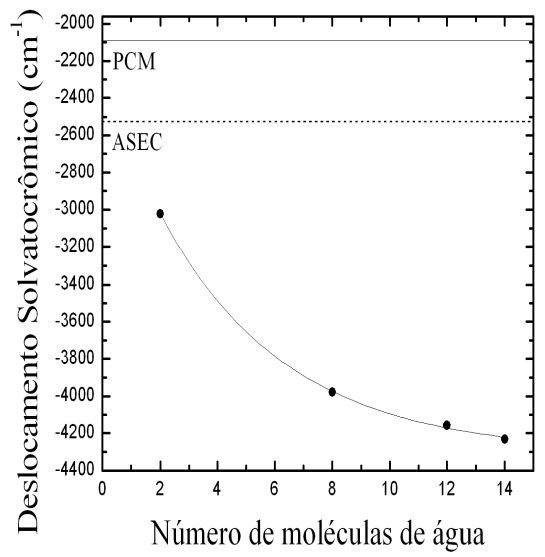

(a) Funcional BHandHLYP

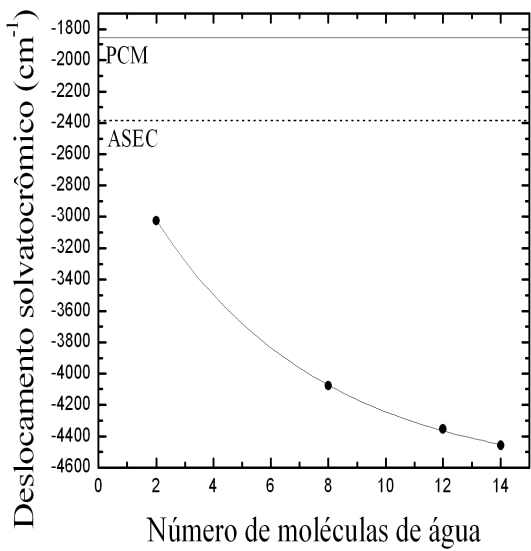

(b) Funcional B3LYP

Figura 4.15: Deslocamento solvatocrômico versus número de águas explícitas. Isômero anti.

$\mathrm{O}$ efeito na mudança syn $\rightarrow$ anti apresenta um deslocamento $\left(\Delta E_{\text {gás }}\right)$ na energia de absorção de $1210 \mathrm{~cm}^{-1}$ e $1713 \mathrm{~cm}^{-1}$, para os funcionais B3LYP e BHandHLYP, respectivamente (obtidos com a função base $6-311++\mathrm{G}^{* *}$ ). O efeito na estrutura eletrônica é apresentado na tabela (4.23). Agora, considerando as duas contribuições nós podemos obter o efeito do solvente sobre o espectro eletrônico de absorção do OM. Na tabela (4.24) apresentamos o valor para o deslocamento $\left(\Delta E_{\text {total }}=\Delta E_{\text {água }}+\Delta E_{\text {gás }}\right)$ levando em conta as duas contribuições para o modelo com um soluto mais 14 águas explicítas.

Tabela 4.24: Efeito do solvente no deslocamento da energia de absorção $\left(\mathrm{cm}^{-1}\right)$ da transição $\pi-\pi^{*}$ do OM. Os valores foram obtidos com TD/DFT usando o conjunto de funções base $6-311++\mathrm{G}^{* *}$.

\begin{tabular}{|c|c|c|c|c|}
\hline \multirow[t]{2}{*}{ Modelo } & \multicolumn{2}{|c|}{$\Delta E_{\text {total }}$} & \multicolumn{2}{|c|}{$\operatorname{Exp} .\left(E_{\text {água }}-E_{\text {iso-octano }}\right)$} \\
\hline & B3LYP & BHandHLYP & B3LYP & BHandHLYP \\
\hline $\mathrm{S}+14 \mathrm{~W}+236 \mathrm{PC}$ & $-3249 \pm 115$ & $-2523 \pm 88$ & \multicolumn{2}{|c|}{$-2150 \pm 350$} \\
\hline
\end{tabular}


Desta forma o nosso melhor deslocamento teórico calculado usando o método TD-DFT é $-2523 \pm 88 \mathrm{~cm}^{-1}$, obtido com o funcional BHandHLYP e considerando 14 moléculas de água de maneira explícita e 236 como cargas pontuais (PC). Esse resultado está em boa concordância com o deslocamento experimental. Observa-se que o funcional B3LYP também descreve a tendência no deslocamento da energia. 


\section{Capítulo 5}

\section{Sumário e Conclusões}

No estudo da molécula isolada nós analisamos três propriedades para verificarmos qual conformação da molécula OM é mais estável. Foram elas: cálculo da energia livre, momento de dipolo e espectro de absorção. Para efeito de comparação nós usamos valores experimentais obtidos com o $\mathrm{OM}$ em iso-octano, um solvente de baixa polaridade. No cálculo teórico da energia livre nós encontramos que em fase gasosa, ou em solventes de baixa polaridade, a molécula de OM deve se encontrar na conformação syn. Esse resultado é corroborado pelos valores experimentais do momento de dipolo e espectro eletrônico de absorção. Em ambos, a conformação syn foi a que apresentou resultados teóricos em melhor concordância comparativamente aos valores experimentais.

Em solução aquosa, para descrever os termos do potencial de interação intermolecular referente a molécula OM ( a molécula não está parametrizada) nós usamos os parâmetros obtidos com o campo de força OPLS. Verificamos que o conjunto de cargas desse campo de força não descreve bem o comportamento eletrostático dessa molécula. Verificamos que o processo de polarização da molécula é uma etapa importante quando se realiza cálculos QM/MM de maneira sequêncial pois observou-se um aumento de aproximadamente $80 \%$ nos dipolos obtidos em fase gasosa.

Neste trabalho realizamos um estudo sobre a energia livre de solvatação. Nessa 
etapa consideramos o solvente através do modelo contínuo PCM e também usando o modelo discreto. O cálculo com modelo contínuo foi feito usando o método MP2/augcc-pVDZ e com o modelo discreto nós usamos teoria de perturbação termodinâmica. Verificamos que a energia livre de solvatação obtida pelo modelo contínuo não descreve a evidência experimental que a molécula $\mathrm{OM}$ é solúvel em água.

Usando teoria de perturbação termodinâmica nós obtivemos a variação de energia livre associada a mudança syn $\rightarrow$ anti. Nós encontramos que o isômero anti deve ser a conformação mais favorável em água, tendo aproximadamente $99 \%$ da concentração total.

Neste trabalho também fizemos o estudo do efeito do solvente no espectro de absorção eletrônica do OM, experimentalmente há resultados que apontam um deslocamento da energia na banda mais intensa provocado pelo solvente, cerca de -2150 $\pm 350 \mathrm{~cm}^{-1}$ (deslocamento água $\rightarrow$ iso-octano), o nosso desafio era tentar descrever quais interações entre o soluto e o solvente estariam provocando esse deslocamento. Para isto nós investigamos a interação soluto-solvente considerando o solvente através do modelo contínuo e também usando o modelo discreto. Os cálculos das energias de absorção foram realizados com método ab initio TD DFT e observou-se que , nos dois modelos para o solvente, a água provoca uma diminuição na energia de absorção, deslocamento para o vermelho, sendo o modelo discreto o que apresenta um deslocamento mais acentuado, porém somente o efeito do solvente na estrutura eletrônica do OM não foi capaz de descrever o deslocamento experimental. Observamos que devido ao fato do OM apresentar uma estabilidade conformacional syn-anti dependente do solvente devemos incluir também a contribuição no deslocamento da energia referente a mudança conformacional. Portanto, de forma resumida, teremos as seguintes contribuições com relação ao efeito do solvente: o efeito na conformação syn-anti, que apresenta um deslocamento para o azul, e o efeito na estrutura eletrônica que apresenta um deslocamento para o vermelho. Desta forma, o nosso melhor resultado para 
o deslocamento foi de $-2523 \pm 88 \mathrm{~cm}^{-1}$, onde $-4232 \mathrm{~cm}^{-1}$ é o efeito do solvente na estrutura eletrônica e $1713 \mathrm{~cm}^{-1}$ é o feito na geometria devido a mudança conformacional. Deslocamento obtido com o funcional BHandHLYP. Entretanto analisando o deslocamento em nm obtemos: $229 \mathrm{~nm}$ (TD-B3LYP/6-311++G**) e $216 \mathrm{~nm}$ (TDBHandHLYP $/ 6-311++\mathrm{G}^{* *}$ ) em gás, $247 \mathrm{~nm}$ (TD-B3LYP/6-311++G**) e $228 \mathrm{~nm}$ (TD-BHandHLYP $/ 6-311++\mathrm{G}^{* *}$ ) em água. Ambos estão em boa concordância com o resultado experimental de $231 \mathrm{~nm}$ em iso-octano e $243 \mathrm{~nm}$ em água [9]. Tendo TD-B3LYP $/ 6-311++\mathrm{G}^{* *}$ obtido uma melhor concordância.

Sendo assim, pretendemos dar continuidade a este trabalho e identificar em que polaridade do solvente existe essa mudança syn-anti na estabilidade conformacional. Adicionalmente analisaremos também a contribuição da flexibilidade interna do OM para o deslocamento da transição eletrônica $\pi-\pi^{*}$, aqui estudada. 


\section{Referências Bibliográficas}

[1] C. Reichardt; Solvent Effects in Organic Chemistry; Verlag Chemie, Weinheim, New York,(1979).

[2] C. Reichardt; Solvatochromic Dyes as Solvent Polarity Indicators; Chem. Rev.94, 2319 (1994).

[3] P. Suppan, N. Ghoneim; Solvatochromism The Royal Society of Chemistry, Londres (1997).

[4] G. C. Maitland, M. Rigby, E. B. Smith, W. A. Wakeham; Intermolecular ForcesTheir Origin and Determination; Oxford University Press, Oxford, (1987).

[5] H. Ratajczak, W. J. Orville-Thomas; Molecular Interactions; vol. 1-3 Wiley, New York, (1980-1982).

[6] S. N. Timasheff; Protein-solvent interactions and protein conformations; Acc. Chem. Res.; 3, 62 (1970).

[7] F. H. Stross, J. M. Monger, H. de V. Finch, The Isolation and Purification of Two Isomers of Mesityl Oxide; J. Am. Chem. Soc. 69, 1627 (1947).

[8] H. F. Gray Jr., R. S. Rasmussen, D. D. Tunnicliff; The Infrared and Ultraviolet Absorption Spectra of Two Isomers of Mesityl Oxide; J. Am. Chem. Soc. 69, 1630 (1947). 
[9] E. M. Kosower; The Effect of Solvent on Spectra; J. Am. Chem. Soc. 80, 3261 (1958).

[10] E. M. Kosower; An Introduction to Physical Organic Chemistry; Wiley, New York (1968).

[11] S. E. Sheppard; The Effects of Environment and Aggregation on the Absorption Spectra of Dyes; Rev. Mod. Phys. 14, 303 (1942).

[12] W. L. Archer; Industrial Solvents Handbook; Dekker, New York, (1996).

[13] J. Franck; Elementary Processes of Photochemical Reactions; Trans. Faraday Soc. 21, 536 (1926).

[14] E. Condon; A Theory of Intensity Distribution in Band Systems; Phys. Rev. 28, $1182(1926)$.

[15] C. Reichardt; Empirical Parameters of the Polarity of Solvent; Angew. Chem. 4, 29 (1965).

[16] P. L. Novaki, O. A. El Soud; Solvatochromism in Binary Solvent Mixtures: Effects of the Molecular Structure of Probe; Ber. Bunsenges. Phys. Chem. 101 $902(1997)$.

[17] A. Streitwieser, C. H. Heathcock, E. M. Kosower; Introduction to Organic Chemistry; McMillan, New York, (1992).

[18] A. D. Buckingham, E. Lippert, E. Bratos, Eds.; Organic Liquids - Structure, Dynamics and Chemical Properties; Wiley, New York, (1978).

[19] K. V. Mikkelsen, H. Ågren, H. J. A. Jensen; A multiconfigurational self-consistent reaction-field method; J. Chem. Phys. 89, 3086 (1988). 
[20] St. Scheiner; Hydrogen Bonding. A Theoretical Perspective; Oxford University Press, New York (1997).

[21] R. Car, M. Parrinello; Unified Approach for Molecular Dynamics and DensityFunctional Theory; Phys. Rev. Lett. 55, 2471 (1985).

[22] R.N. Barnett, U. Landman; Born-Oppenheimer molecular-dynamics simulations of finite systems: Structure and dynamics of $\left(\mathrm{H}_{2} \mathrm{O}\right)_{2}$; Phys. Rev. B48, 2081 (1993).

[23] Hans Martin Senn, Walter Thiel; QM/MM Methods for Biological Systems; Top. Curr. Chem.; Springer-Verlag, Berlim (2006).

[24] K. Coutinho, S. Canuto, Solvent effects from a sequential Monte Carlo - quantum mechanical approach; Adv. Quantum Chem. 28, 89 (1997).

[25] K. Coutinho, S. Canuto, M. C. Zerner; Calculation of the Absorption Spectrum of Benzene in Condensed Phase. A Study of the Solvent Effects; Int. J. Quantum Chem.65, 885 (1997).

[26] J. D. M. Vianna, A. Fazzio, S. Canuto; Teoria Quântica de Moléculas e Sólidos; Livraria da Física, São Paulo (2004).

[27] M. Born, R. Oppenheimer; Quantum theory of molecules; Ann. Phys. Leipzig. 84, 457 (1927).

[28] M. Born, K. Huang; Dynamical Theory of Cristal Lattice; Oxford University Press, N. Y. apêndice VII (1954).

[29] D. R Yarkony; Encyclopedia of Computational Chemistry; John Wiley \& Sons, New York (1998).

[30] D. R. Hartree; The Wave Mechanics of an Atom with a Non-Coulomb Central Field. Part I-Theory and Methods; Proc. Cambridge Phil. Soc. 24, 89 (1928). 
[31] D. R. Hartree; The Wave Mechanics of an Atom with a Non-Coulomb Central Field.Part II-Some Results and Discussion; Proc. Cambridge Phil. Soc. 24, 111 (1928).

[32] A. Szabo, N. S. Ostlund;Modern Quantum Chemistry; Dover, New York (1996).

[33] M. W. Hanna; Quantum Mechanics in Chemistry; $2^{\mathrm{a}}$ Ed., W. A. Benjamin Inc., New York, (1969).

[34] J. C. Slater; The Theory of Complex Spectra; Phys. Rev. 34, 1293 (1929).

[35] N. H. Morgon, K. Coutinho (Eds); Métodos de Química Teórica e Modelagem Molecular; Livraria da Física, São Paulo (2007).

[36] H. Goldstein; Classical Mechanics, Addison-Wesley, London

[37] I. N. Levine; Quantum Chemistry; 5a Ed., Prentice Hall, New Jersey (2000).

[38] C. C. J. Roothaan; New Developments in Molecular Orbital Theory, Rev. Mod. Phys. 23, 69 (1951).

[39] C. Froese-Fisher; The Hartree-Fock Methods for Atoms; $1^{\text {a }}$ Ed., John Wiley \& Sons, New York (1977).

[40] P. O. Löwdin; Correlation problem in many-electron quantum mechanics. I. Review of different approaches and discussion of some current ideas; Adv. Chem. Phys. 2, 207 (1959).

[41] S. J. Huzinaga; Gaussian-Type Functions for Polyatomic Systems. I; J. Chem. Phys. 42, 1293 (1965).

[42] W. J. Hehre, L. Randon, P. V. R. Schleyer, J. A. Pople; Ab initio Molecular Orbital Theory; Jonh Wiley \& Sons, New York, (1996). 
[43] R. Krishnan, J. S. Binkley, R. Seeger, J. A. Pople; Self-consistent molecular orbital methods. XX. A basis set for correlated wave functions; J. Chem. Phys. 72, 650 (1980).

[44] M. J. Frisch, G. W. Trucks, H. B. Schlegel, G. E. Scuseria, M. A. Robb, J. R. Cheeseman, J. A. Montgomery, Jr., T. Vreven, K. N. Kudin, J. C. Burant, J. M. Millam, S. S. Iyengar, J. Tomasi, V. Barone, B. Mennucci, M. Cossi, G. Scalmani, N. Rega, G. A. Petersson, H. Nakatsuji, M. Hada, M. Ehara, K. Toyota, R. Fukuda, J. Hasegawa, M. Ishida, T. Nakajima, Y. Honda, O. Kitao, H. Nakai, M. Klene, X. Li, J. E. Knox, H. P. Hratchian, J. B. Cross, V. Bakken, C. Adamo, J. Jaramillo, R. Gomperts, R. E. Stratmann, O. Yazyev, A. J. Austin, R. Cammi, C. Pomelli, J. W. Ochterski, P. Y. Ayala, K. Morokuma, G. A. Voth, P. Salvador, J. J. Dannenberg, V. G. Zakrzewski, S. Dapprich, A. D. Daniels, M. C. Strain, O. Farkas, D. K. Malick, A. D. Rabuck, K. Raghavachari, J. B. Foresman, J. V. Ortiz, Q. Cui, A. G. Baboul, S. Clifford, J. Cioslowski, B. B. Stefanov, G. Liu, A. Liashenko, P. Piskorz, I. Komaromi, R. L. Martin, D. J. Fox, T. Keith, M. A. Al-Laham, C. Y. Peng, A. Nanayakkara, M. Challacombe, P. M. W. Gill, B. Johnson, W. Chen, M. W. Wong, C. Gonzalez, and J. A. Pople, Gaussian 03, Revision C.02, Gaussian, Inc., Wallingford CT (2004).

[45] C.Møller, M. S. Plesset; Note on an Approximation Treatment for Many-Electron Systems; Phys. Rev. 46, 618 (1934).

[46] R. Krishnan, J. A. Pople; Approximate fourth-order perturbation theory of the electron correlation energy; Int. J. Quantum Chem. 14, 91 (1978).

[47] J. Olsen, O. Christiansen, H. Koch, P. Jorgensen; Surprising cases of divergent behavior in Møller-Plesset perturbation theory; J. Chem. Phys. 105, 5082 (1996).

[48] B. H. Brandow; Linked-Cluster Expansions for the Nuclear Many-Body Problem; Rev. Mod. Phys. 39, 771 (1967). 
[49] P. Hohenberg, W. Kohn; Inhomogeneous Electron Gas; Phys. Rev. B 136, B864 (1964).

[50] W. Kohn, L. J. Sham; Self-consistent equations including exchange and correlation effects; Phys. Rev. 140, 1133 (1965)

[51] J. P. Perdew, K. Burke; Comparison shopping for a gradient-corrected density functional; Int. Quantum Chem. 57, 309 (1996).

[52] J. P. Perdew, K. Burke, M. Ernzenhof; Generalized Gradient Approximation Made Simple; Phys. Rev. Lett. 77, 3865 (1996); 78, 1396 (1997).

[53] C. Lee, W. Yang, R. G. Parr; Development of the Colle-Salvetti correlationenergy formula into a functional of the electron density; Phys. Rev. B 37, 785 (1988)

[54] A. D. Becke; Density-functional thermochemistry. III. The role of exact exchange; J. Chem. Phys. 98, 5648 (1993).

[55] J. P. Perdew, Y. Wang; Accurate and simple density functional for the electronic exchange energy: Generalized gradient approximation; Phys. Rev. B 33, 8800 (1986).

[56] J. P. Perdew; Density-functional approximation for the correlation energy of the inhomogeneous electron gas; Phys. Rev. B 33, 8822 (1986).

[57] A. D. Becke; Density-functional exchange-energy approximation with correct asymptotic behaviour; Phys. Rev. A 38, 3098 (1988).

[58] J. P. Perdew, K. Burke, Y. Wang; Generalized gradient approximation for the exchange-correlation hole of a many-electron system; Phys. Rev. B 54, 16533 (1996). 
[59] Y. Imamura, T. Otsuka, H. Nakai; Description of core excitations by timedependent density functional theory with local density approximation, generalized gradient approximation, meta-generalized gradient approximation, and hybrid functionals; J. Comput. Chem. 28, 2067 (2007).

[60] A.D. Becke; A new mixing of Hartree-Fock and local density-functional theories; J. Chem. Phys. 98, 1372 (1993).

[61] S. H. Vosko, L. Wilk, M. Nusair; Accurate spin-dependent electron liquid correlation energies for local spin density calculations: a critical analysis; Can. J. Phys. 58, 1200 (1980).

[62] M. Horbatsch, R. M. Dreizler; Time-dependent Thomas-Fermi approach to atomic collisions I. High-energy proton-atom scattering; Z. Phys. A 300, 119 (1992).

[63] G. Holzwarth; Static and dynamical Thomas Fermi theory for nuclei; Phys. Lett. B 66, 29 (1977).

[64] P. Malzacher, R. M. Dreizler; Charge oscillations and photoabsorption of the Thomas-Fermi-Dirac-Weizsäcker atom; Z. Phys. A 307, 211 (1982).

[65] E. Runge, E. K. U. Gross; Density-Functional Theory for Time-Dependent Systems; Phys. Rev. Lett 52, 997 (1984).

[66] M. A. L. Marques, E. K. U. Gross; Time-dependent density functional theory; Annu. Rev. Phys. Chem. 55, 427 (2004).

[67] R. S. Mulliken; Electronic Population Analysis on LCAO[Single Bond]MO Molecular Wave Functions. I; J. Chem. Phys. 23, 1833 (1955).

[68] K. B. Wiberg, P. R. Rablen; A Comparison of Atomic Charges Derived via Different Procedures; J. Comp. Chem.14, 1504 (1993). 
[69] D.E. Williams, J. Yan; Adv. Atomic Mol. Phys. 23, 87 (1988).

[70] F. A. Momany; Determination of partial atomic charges from ab initio molecular electrostatic potentials. Application to formamide, methanol, and formic acid; J. Phys. Chem. 82, 592 (1978).

[71] S. R. Cox, D.E. Williams; Representation of the molecular electrostatic potential by a net atomic charge model; J. Comp. Chem. 2, 304 (1981).

[72] C. M. Breneman, K. B. Wiberg; Determining atom-centered monopoles from molecular electrostatic potentials. The need for high sampling density in formamide conformational analysis; J. Comp. Chem. 11, 361 (1990).

[73] U. C. Singh, P. A. Kollman; An approach to computing electrostatic charges for molecules; J. Comp. Chem. 5, 129 (1984).

[74] M. Born; Volumes and heats of hydration of ions; Z. Phys. 1, 45 (1920).

[75] J. G. Kirkwood; Theory of solutions of molecules containing widely separated charges with special application to amphoteric ions; J. Chem. Phys. 2, 351 (1934).

[76] L. Onsager; Electric Moments of Molecules in Liquids; J. Am. Chem. Soc. 58, $1486(1936)$.

[77] C. Böttcher; Theory of Electronic Polarization; Elsevier, Amsterdan, (1973).

[78] S. Miertus, E. Scrocco, J. Tomasi; Electrostatic Interaction of a Solute with a Continuum. A Direct Utilization of ab initio Molecular Potentials for the Prevision of Solvent Effects; J. Chem. Phys. 55, 117 (1981).

[79] J. Tomasi, R. Cammi e B. Mennuci. Medium Effects on the Properties of Chemical Systems: an Overview of Recent Reformulations in the Polarizable Continuum Model (PCM); Int. J. Quantum Chem. 75, 783 (1999). 
[80] Sílvio R. A. Salinas; Introdução à Física Estatistica; Editora da Universidade de São Paulo, São Paulo (2005).

[81] J. P. Hansen, I. R. McDonald; Theory of Simple Liquids; Academic Press, London (1986).

[82] T. E. Hill; An Introduction to Statistical Mechanics; Dover, New York (1986).

[83] M. P. Allen, D. J. Tildesley; Computer Simulation of Liquids; Oxford University Press, Oxford, (1987).

[84] P. Ehrenfest, T. Ehrenfest; The Conceptual Foundations of the Statistical Approach in Mechanics; Dover, New York, (1990).

[85] N. Metropolis; A. W. Rosenbluth; M. N. Rosenbluth; A. H. Teller, E. Teller; Equations of State Calculations by Fast Computing Machines; J. Chem. Phys. 21, 1087 (1953).

[86] H. C. Georg; Efeitos do Meio em Propriedades Conformacionais e Eletrônicas de Moléculas; tese de doutoramento, Universidade de São Paulo, São Paulo (2006).

[87] K. Coutinho, S. Canuto, DICE: Um programa para simulação computacional de líquidos moleculares, utilizando o método Monte Carlo Metropolis e Teoria da Perturbação Termodinâmica para cálculos de energia livre; Universidade de São Paulo (1997).

[88] K. Coutinho; Modelo Discreto de Solvente. Solvatocromismo no Espectro de Absorção Molecular; tese de doutoramento, Universidade de São Paulo, São Paulo (1997).

[89] B. M. Axilrol, E. Teller; J. Chem. Phys. 11, 299 (1943).

[90] J. E. Lennard-Jones; Physica 4, 957 (1937). 
[91] C. Chatfield; The Analysis of Time Series. An Introduction; Chapman and Hall, London, $3^{\text {a }}$ Ed., (1984).

[92] P. Kollman; Free energy calculations: Applications to chemical and biochemical phenomena; Chem. Rev. 93, 2395 (1993).

[93] C. H. Bennett; Efficient Estimation of Free Energy Differences from Monte Carlo Data; J. Comp. Phys. 22, 245 (1976).

[94] P. A. Bash, U. C. Singh, R. Langridge, P. Kollman; Free energy calculations by computer simulation; Science 236, 564 (1987).

[95] R. W. Zwanzig; High-Temperature Equation of State by a Perturbation Method. I. Nonpolar Gases; J. Chem. Phys. 22, 1420 (1954).

[96] W. L. Jorgensen, J. K. Buckner, S. Boudon, J. Tirado-Rives; Efficient computation of absolute free energies of binding by computer simulations. Application to the methane dimer in water; J. Chem. Phys. 89, 3742 (1988).

[97] Herbert C. Georg, K. Coutinho, S. Canuto; A look inside the cavity of hydrated Q-cyclodextrin: A computer simulation study; Chem. Phys. Lett. 413, 16 (2005).

[98] L. C. G. Freitas, L. B. Silva, L. F. Botelho; Termodinâmica estatística de líquidos com o método de Monte Carlo. I. Metodologia; Quím. Nova. 19, 166 (1996).

[99] W. L. Jorgensen, J. F. Blake, J. K. Buckner; Free energy of TIP4P water and the free energies of hidration of $\mathrm{CH}_{4}$ and $\mathrm{Cl}^{-}$from statistical perturbation theory; Chem. Phys. 129, 193 (1989).

[100] W. L. Jorgensen, D. L. Severance; Aromatic-aromatic interactions: free energy profiles for the benzene dimer in water, chloroform, and liquid benzene; J. Am. Chem. Soc. 112, 4768 (1990). 
[101] H. J. C. Berendsen, J. P. M. Postma, W. F. van Gunsteren, and J. Hermans; Intermolecular Forces; edited by B. Pullman, Reidel, Dordrecht (1981).

[102] W. L. Jorgensen, D. S. Maxwell, J. Tirado-Rives; Development and Testing of the OPLS All-Atom Force Field on Conformational Energetics and Properties of Organic Liquids; J. Am. Chem. Soc. 118, 11225 (1996) e informatic support.

[103] C. M. Breneman, K. B. Wiberg; Determining atom-centered monopoles from molecular electrostatic potentials. The need for high sampling density in formamide conformational analysis; J. Comp. Chem. 11, 361 (1990).

[104] M. Mezei, D. L. Beveridge; Theoretical studies of hydrogen bonding in liquid water and aqueous solution; J. Chem. Phys. 74, 622 (1981).

[105] M. G. Sceats, S. A. Rice; The water-water pair potential near the hydrogen bonded equilibrium configuration; J. Chem. Phys. 72, 3236 (1981).

[106] K. Coutinho, S. Canuto; The sequential Monte Carlo-quantum Mechanics Methodology. Application to the Solvent Effects in the Stokes Shift of Acetone in Water; J. Mol. Struct. (Theochem) 632, 235 (2003).

[107] H. C. Georg, K. Coutinho, S. Canuto; A sequential Monte Carlo quantum mechanics study of the hydrogen-bond interaction and the solvatochromic shift of the $n-\pi^{*}$ transition of acrolein in water; J. Chem. Phys. 123, 124307 (2005).

[108] H. C. Georg, K. Coutinho, S. Canuto;Polarization and Solvatochromic shift of orto-betaine in water; Chem. Phys. Lett.429, 119 (2006).

[109] K. Coutinho, H. C. Georg, T. L. Fonseca, V. Ludwig, S. Canuto; An efficient statistically converged average configuration for solvent effects; Chem. Phys. Lett. 437, 148 (2007). 
[110] W. F. V. Gunsteren, P. K. Weiner, (ed.); Computer Simulation of Biomolecular Systems: Theoretical and Experimental Aplications; ESCOM, Leiden (1989). 


\section{Apêndice A}

\section{Conjunto de Cargas Atômicas}

Nas tabelas A.1 e A.2 apresentamos os conjuntos de cargas para o isômero syn e para o estado de transição TS obtidos pelos diferentes procedimentos: i) gás - cargas obtidas realizando cálculo quântico na molécula isolada no nível MP2/aug-cc-pVDZ, ii) OPLS - obtido pelo campo de força, iii) PCM - obtido realizando cálculo quântico com o soluto num meio contínuo polarizável, iv) iterativo - obtido pelo processo de polarização descrito na seção 4.2.4. 
Tabela A.1: Teste do potencial eletrostático para o isômero syn.

\begin{tabular}{lcccc}
\hline \hline Átomo & gás & OPLS & PCM & iterativo \\
\hline C1 & 0.393 & 0.000 & 0.416 & 0.456 \\
C2 & -0.585 & -0.115 & -0.618 & -0.634 \\
C3 & 0.746 & 0.500 & 0.790 & 0.868 \\
O4 & -0.538 & -0.500 & -0.620 & -0.753 \\
C5 & -0.341 & -0.180 & -0.372 & -0.377 \\
C6 & -0.309 & -0.180 & -0.344 & -0.344 \\
C7 & -0.326 & -0.180 & -0.335 & -0.342 \\
H8 & 0.156 & 0.115 & 0.187 & 0.182 \\
H9 & 0.085 & 0.060 & 0.106 & 0.115 \\
H10 & 0.085 & 0.060 & 0.106 & 0.116 \\
H11 & 0.084 & 0.060 & 0.083 & 0.088 \\
H12 & 0.083 & 0.060 & 0.097 & 0.105 \\
H13 & 0.087 & 0.060 & 0.101 & 0.099 \\
H14 & 0.087 & 0.060 & 0.101 & 0.108 \\
H15 & 0.080 & 0.060 & 0.088 & 0.095 \\
\hline \hline & 0.133 & 0.060 & 0.124 & 0.124 \\
H16 & 0.080 & 0.060 & 0.088 & 0.092 \\
Hipolo(D) & 2.77 & 3.63 & 3.85 & 4.97 \\
\hline
\end{tabular}


Tabela A.2: Teste do potencial eletrostático para o estado de transição (TS).

\begin{tabular}{lcccc}
\hline \hline Átomo & gás & OPLS & PCM & iterativo \\
\hline C1 & 0.239 & 0.000 & 0.250 & 0.261 \\
C2 & -0.550 & -0.115 & -0.604 & -0.608 \\
C3 & 0.840 & 0.500 & 0.893 & 0.968 \\
O4 & -0.553 & -0.500 & -0.643 & -0.754 \\
C5 & -0.381 & -0.180 & -0.365 & -0.383 \\
C6 & -0.216 & -0.180 & -0.228 & -0.212 \\
C7 & -0.162 & -0.180 & -0.179 & -0.189 \\
H8 & 0.168 & 0.115 & 0.203 & 0.194 \\
H9 & 0.086 & 0.060 & 0.097 & 0.116 \\
H10 & 0.085 & 0.060 & 0.097 & 0.114 \\
H11 & 0.098 & 0.060 & 0.090 & 0.095 \\
H12 & 0.066 & 0.060 & 0.072 & 0.074 \\
H13 & 0.055 & 0.060 & 0.069 & 0.074 \\
H14 & 0.071 & 0.060 & 0.073 & 0.064 \\
H15 & 0.046 & 0.060 & 0.064 & 0.077 \\
\hline \hline
\end{tabular}




\section{Apêndice B}

\section{Parâmetros Usados na Simulação.}

Nas tabelas a seguir apresentamos os dados usados para a simulação de cada isômero. Cada átomo é caracterizado pelo seu número atômico, pelas coordenadas xyz, pela sua carga, e pelos parâmetros $\epsilon$ e $\sigma$ do potencial de interação intermolecular. Aqui, o conjunto de cargas é o obtido pelo processo de polarização e os parâmetros $\epsilon$ e $\sigma$ foram obtidos do campo de força OPLS. O modelo utilizado para a água foi o SPC 101. 
Tabela B.1: Tabela com os parâmetros necessários para realizar uma simulação no DICE. Isômero syn

\begin{tabular}{|c|c|c|c|c|c|c|}
\hline \multirow{2}{*}{$\frac{\text { Átomo }}{6}$} & \multicolumn{3}{|c|}{ Coordenadas xyz } & \multirow{2}{*}{$\begin{array}{l}\text { Cargas } \\
0.456022\end{array}$} & \multirow{2}{*}{$\frac{\epsilon}{0.066}$} & \multirow{2}{*}{$\frac{\sigma}{3.500}$} \\
\hline & 1.209578 & -0.121181 & 0.000021 & & & \\
\hline 6 & -0.042280 & -0.638221 & -0.000128 & -0.633782 & 0.066 & 3.500 \\
\hline 6 & -1.336879 & 0.088455 & -0.000324 & 0.868422 & 0.066 & 3.500 \\
\hline 8 & -1.452069 & 1.310750 & 0.000036 & -0.752795 & 0.210 & 2.960 \\
\hline 6 & -2.573179 & -0.800785 & 0.000089 & -0.377144 & 0.066 & 3.500 \\
\hline 6 & 2.403675 & -1.043505 & 0.000113 & -0.343849 & 0.066 & 3.500 \\
\hline 6 & 1.543141 & 1.346830 & 0.000023 & -0.341809 & 0.066 & 3.500 \\
\hline 1 & -0.136863 & -1.723958 & -0.000098 & 0.182165 & 0.030 & 2.500 \\
\hline 1 & -2.576939 & -1.454590 & -0.882127 & 0.115358 & 0.030 & 2.500 \\
\hline 1 & -2.576776 & -1.453976 & 0.882767 & 0.116423 & 0.030 & 2.500 \\
\hline 1 & -3.473095 & -0.182185 & -0.000023 & 0.088589 & 0.030 & 2.500 \\
\hline 1 & 2.117018 & -2.099639 & 0.000401 & 0.104929 & 0.030 & 2.500 \\
\hline 1 & 3.034628 & -0.855077 & 0.879873 & 0.099478 & 0.030 & 2.500 \\
\hline 1 & 3.034405 & -0.855513 & -0.879906 & 0.107511 & 0.030 & 2.500 \\
\hline 1 & 2.157814 & 1.587147 & 0.879404 & 0.095092 & 0.030 & 2.500 \\
\hline 1 & 0.654197 & 1.975082 & 0.000007 & 0.123547 & 0.030 & 2.500 \\
\hline 1 & 2.157819 & 1.587143 & -0.879358 & 0.091841 & 0.030 & 2.500 \\
\hline
\end{tabular}


Tabela B.2: Tabela com os parâmetros necessários para realizar uma simulação no DICE. Estado de transição $T S$

\begin{tabular}{|c|c|c|c|c|c|c|}
\hline Átomo & $\mathrm{Co}$ & rdenadas $\mathrm{x}$ & & Cargas & $\epsilon$ & $\sigma$ \\
\hline 6 & 1.179799 & -0.038597 & 0.016086 & 0.260744 & 0.066 & 3.500 \\
\hline 6 & 0.055108 & -0.767123 & 0.078765 & -0.608218 & 0.066 & 3.500 \\
\hline 6 & -1.343197 & -0.251277 & -0.142032 & 0.968154 & 0.066 & 3.500 \\
\hline 8 & -1.872144 & -0.308296 & -1.240375 & -0.754167 & 0.210 & 2.960 \\
\hline 6 & -2.077952 & 0.286615 & 1.071129 & -0.383392 & 0.066 & 3.500 \\
\hline 6 & 2.537312 & -0.668225 & 0.216220 & -0.212238 & 0.066 & 3.500 \\
\hline 6 & 1.192323 & 1.442939 & -0.277388 & -0.188963 & 0.066 & 3.500 \\
\hline 1 & 0.112804 & -1.839219 & 0.274172 & 0.194540 & 0.030 & 2.500 \\
\hline 1 & -1.523917 & 1.127585 & 1.507223 & 0.115766 & 0.030 & 2.500 \\
\hline 1 & -2.129069 & -0.488003 & 1.847928 & 0.114545 & 0.030 & 2.500 \\
\hline 1 & -3.087451 & 0.603854 & 0.796789 & 0.095217 & 0.030 & 2.500 \\
\hline 1 & 2.465355 & -1.739735 & 0.428212 & 0.074089 & 0.030 & 2.500 \\
\hline 1 & 3.072879 & -0.188866 & 1.047654 & 0.074056 & 0.030 & 2.500 \\
\hline 1 & 3.162733 & -0.537720 & -0.677875 & 0.064110 & 0.030 & 2.500 \\
\hline 1 & 1.678706 & 1.997533 & 0.537161 & 0.076621 & 0.030 & 2.500 \\
\hline 1 & 0.191381 & 1.858071 & -0.427824 & 0.041825 & 0.030 & 2.500 \\
\hline 1 & 1.773368 & 1.646871 & -1.187118 & 0.067311 & 0.030 & 2.500 \\
\hline
\end{tabular}


Tabela B.3: Tabela com os parâmetros necessários para realizar uma simulação no DICE. Isômero anti.

\begin{tabular}{|c|c|c|c|c|c|c|}
\hline Átomo & \multicolumn{3}{|c|}{ Coordenadas xyz } & Cargas & $\epsilon$ & $\sigma$ \\
\hline 6 & 1.186200 & -0.066353 & -0.000104 & 0.301804 & 0.066 & 3.500 \\
\hline 6 & 0.004992 & -0.727124 & 0.000127 & -0.547855 & 0.066 & 3.500 \\
\hline 6 & -1.410503 & -0.275840 & -0.000048 & 0.837366 & 0.066 & 3.500 \\
\hline 8 & -2.282194 & -1.142390 & -0.000485 & -0.768622 & 0.210 & 2.960 \\
\hline 6 & -1.826392 & 1.186385 & 0.000542 & -0.310822 & 0.066 & 3.500 \\
\hline 6 & 2.469464 & -0.865902 & 0.000301 & -0.168548 & 0.066 & 3.500 \\
\hline 6 & 1.416942 & 1.423868 & -0.000512 & -0.318932 & 0.066 & 3.500 \\
\hline 1 & 0.046002 & -1.815223 & 0.000450 & 0.172740 & 0.030 & 2.500 \\
\hline 1 & -1.448515 & 1.709058 & -0.886015 & 0.101599 & 0.030 & 2.500 \\
\hline 1 & -1.446606 & 1.708995 & 0.886311 & 0.098655 & 0.030 & 2.500 \\
\hline 1 & -2.917863 & 1.230065 & 0.001621 & 0.085857 & 0.030 & 2.500 \\
\hline 1 & 2.285361 & -1.943856 & 0.001739 & 0.050522 & 0.030 & 2.500 \\
\hline 1 & 3.079794 & -0.618417 & 0.880163 & 0.073203 & 0.030 & 2.500 \\
\hline 1 & 3.078888 & -0.620630 & -0.880808 & 0.072375 & 0.030 & 2.500 \\
\hline 1 & 2.009933 & 1.707875 & 0.880054 & 0.110545 & 0.030 & 2.500 \\
\hline 1 & 0.510345 & 2.024556 & -0.004095 & 0.099435 & 0.030 & 2.500 \\
\hline 1 & 2.015986 & 1.706490 & -0.877381 & 0.110679 & 0.030 & 2.500 \\
\hline
\end{tabular}


Tabela B.4: Parâmetros obtidos do modelo SPC para a água [101].

\begin{tabular}{|c|c|c|c|c|c|c|}
\hline Átomo & & \multicolumn{2}{|c|}{ Coordenadas xyz } & Cargas & $\epsilon$ & $\sigma$ \\
\hline 8 & 0.0000 & 0.0000 & 0.0000 & -0.820 & 0.1550 & 3.1650 \\
\hline 1 & 0.5774 & 0.8165 & 0.0000 & 0.410 & 0.0000 & 0.0000 \\
\hline 1 & 0.5774 & -0.8165 & 0.0000 & 0.410 & 0.0000 & 0.0000 \\
\hline
\end{tabular}

